\title{
Synchronization in Complex Networks of Phase Oscillators: A Survey
}

\author{
Florian Dörfler ${ }^{\mathrm{a}}$, Francesco Bullo ${ }^{\mathrm{b}}$ \\ ${ }^{a}$ Department of Electrical Engineering, University of California Los Angeles, USA \\ ${ }^{\mathrm{b}}$ Department of Mechanical Engineering, University of California Santa Barbara, USA
}

\begin{abstract}
The emergence of synchronization in a network of coupled oscillators is a fascinating subject of multidisciplinary research. This survey reviews the vast literature on the theory and the applications of complex oscillator networks. We focus on phase oscillator models that are widespread in real-world synchronization phenomena, that generalize the celebrated Kuramoto model, and that feature a rich phenomenology. We review the history and the countless applications of this model throughout science and engineering. We justify the importance of the widespread coupled oscillator model as a locally canonical model and describe some selected applications relevant to control scientists, including vehicle coordination, electric power networks, and clock synchronization. We introduce the reader to several synchronization notions and performance estimates. We propose analysis approaches to phase and frequency synchronization, phase balancing, pattern formation, and partial synchronization. We present the sharpest known results about synchronization in networks of homogeneous and heterogeneous oscillators, with complete or sparse interconnection topologies, and in finite-dimensional and infinite-dimensional settings. We conclude by summarizing the limitations of existing analysis methods and by highlighting some directions for future research.
\end{abstract}

\section{Introduction}

Synchronization in networks of coupled oscillators is a pervasive topic in various scientific disciplines ranging from biology, physics, and chemistry to social networks and technological applications. A coupled oscillator network is characterized by a population of heterogeneous oscillators and a graph describing the interaction among the oscillators. These two ingredients give rise to a rich dynamic behavior that keeps on fascinating the scientific community.

Within the rich modeling phenomenology on synchronization among coupled oscillators, this article focuses on the widely adapted model of a continuous-time and periodic limit-cycle oscillator network with continuous, bidirectional, and antisymmetric coupling. We consider a system of $n$ oscillators, each characterized by a phase angle $\theta_{i} \in \mathbb{S}^{1}$ and a natural rotation frequency $\omega_{i} \in \mathbb{R}$. The dynamics of each isolated oscillator are thus $\dot{\theta}_{i}=\omega_{i}$

\footnotetext{
* This material is based in part upon work supported by UCLA startup funds and NSF grants IIS-0904501 and CPS1135819. A preliminary short version of this document appeared as (Dörfler and Bullo, 2012a).

Email addresses: dorfler@seas.ucla.edu (Florian Dörfler), bullo@engineering.ucsb.edu (Francesco Bullo).
}

for $i \in\{1, \ldots, n\}$. The interaction topology and coupling strength among the oscillators are modeled by a connected, undirected, and weighted graph $G=(\mathcal{V}, \mathcal{E}, A)$ with nodes $\mathcal{V}=\{1, \ldots, n\}$, edges $\mathcal{E} \subset \mathcal{V} \times \mathcal{V}$, and positive weights $a_{i j}=a_{j i}>0$ for each undirected edge $\{i, j\} \in \mathcal{E}$. The interaction between neighboring oscillators is assumed to be additive, anti-symmetric, diffusive, ${ }^{1}$ and proportional to the coupling strengths $a_{i j}$. In this case, the simplest $2 \pi$-periodic interaction function between neighboring oscillators $\{i, j\} \in \mathcal{E}$ is $a_{i j} \sin \left(\theta_{i}-\theta_{j}\right)$, and the overall model of coupled phase oscillators reads as

$$
\dot{\theta}_{i}=\omega_{i}-\sum_{j=1}^{n} a_{i j} \sin \left(\theta_{i}-\theta_{j}\right), \quad i \in\{1, \ldots, n\} .
$$

Despite its apparent simplicity, this coupled oscillator model gives rise to rich dynamic behavior, and it is encountered in many scientific disciplines ranging from natural and life sciences to engineering. This article surveys recent results and applications of the coupled oscillator model (1) and of its variations.

The motivations for this survey are manifold. Recent

\footnotetext{
1 The interaction between two oscillators is diffusive if its strength depends on the corresponding phase difference; such interactions arise for example in the discretization of the Laplace operator in diffusive partial differential equations.
} 
years have witnessed much theoretical progress and novel applications, which are not covered in existing surveys (Strogatz, 2000; Acebrón et al., 2005; Arenas et al., 2008; Dorogovtsev et al., 2008) published in the physics literature. Indeed, control scientists have shown an increasing interest in complex networks of coupled oscillators and have recently contributed many novel approaches and results. Much of this interest has focused on (i) synchronization rather than more complex dynamic phenomena, (ii) finite numbers of oscillators with a non-trivial interaction topology, and (iii) connections with graph theory and multi-agent systems. It is therefore timely to provide a comprehensive review in a unified control-theoretical language of the best known results in this area. With this aim, this survey provides a systems and control perspective to coupled oscillator networks, focusing on quantitative results and control-relevant applications in sciences and technology.

\subsection{Mechanical Analog and Basic Phenomenology}

A mechanical analog of the coupled oscillator model (1) is the spring network shown in Fig. 1. This network consists of a group of particles constrained to move on a unit circle and assumed to move without colliding. Each particle is characterized by its angle $\theta_{i} \in \mathbb{S}^{1}$ and

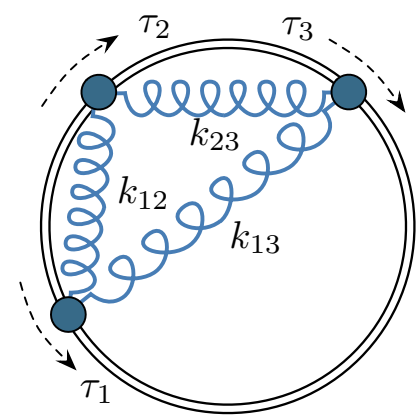

Fig. 1. Mechanical analog of a coupled oscillator network

frequency $\dot{\theta}_{i} \in \mathbb{R}$, and its inertial and damping coefficients are $M_{i}>0$ and $D_{i}>0$. Pairs of interacting particles $i$ and $j$ are coupled through a linear-elastic spring with stiffness $k_{i j}>0$. The external forces and torques acting on each particle are a viscous damping force $D_{i} \dot{\theta}_{i}$ opposing the direction of motion, an external driving torque $\tau_{i} \in \mathbb{R}$, and an elastic restoring torque $k_{i j} \sin \left(\theta_{i}-\theta_{j}\right)$ between pairs of interacting particles. The overall spring network is modeled by a graph, whose nodes are the particles, whose edges are the linear-elastic springs, and whose edge weights are the positive stiffness coefficients $k_{i j}=k_{j i}$. Under these assumptions, it can be shown (Dörfler et al., 2013) that the system of spring-interconnected particles obeys the dynamics

$$
M_{i} \ddot{\theta}_{i}+D_{i} \dot{\theta}_{i}=\tau_{i}-\sum_{j=1}^{n} k_{i j} \sin \left(\theta_{i}-\theta_{j}\right), i \in\{1, \ldots, n\} .
$$

In the limit of small masses $M_{i}$ and uniformly-high viscous damping $D=D_{i}$, that is, $M_{i} / D \approx 0$, we recover the coupled oscillator dynamics (1) from its mechanical analog (2) with natural rotation frequencies $\omega_{i}=\tau_{i} / D$ and with coupling strenghts $a_{i j}=k_{i j} / D$.

The mechanical analog in Fig. 1 illustrates the basic phenomenology displayed by the oscillator network (1). The spring-interconnected particles are subject to a competition between the external driving forces $\omega_{i}$ and the internal restoring torques $a_{i j} \sin \left(\theta_{i}-\theta_{j}\right)$. Hence, the interesting coupled oscillator dynamics (1) arise from a tradeoff between each oscillator's tendency to align with its natural frequency $\omega_{i}$ and the synchronization-enforcing coupling $a_{i j} \sin \left(\theta_{i}-\theta_{j}\right)$ with its neighbors. Intuitively, a weakly coupled and strongly heterogeneous (i.e., with strongly dissimilar natural frequencies) network does not display any coherent behavior, whereas a strongly coupled and sufficiently homogeneous network is amenable to synchronization, where all frequencies $\dot{\theta}_{i}(t)$ or even all phases $\theta_{i}(t)$ become aligned.

\subsection{History, Related Applications, and Theoretical De- velopments:}

A brief history of synchronization: The scientific interest in synchronization of coupled oscillators can be traced back to the work by Huygens (1893) on "an odd kind of sympathy" between coupled pendulum clocks, locking phenomena in circuits and radio technology (Appleton, 1922; Van Der Pol, 1927; Adler, 1946), mutual influence of organ pipes (Rayleigh, 1896), the analysis of brain waves and self-organizing systems (Wiener, 1948, 1958), and it still fascinates the scientific community nowadays (Winfree, 2001; Strogatz, 2003). We refer to Pikovsky et al. (2003) and Blekhman (1988) for a detailed historical account of synchronization studies.

A variation of the considered coupled oscillator model (1) was first proposed by Winfree (1967). Winfree considered general (not necessarily sinusoidal) interactions among the oscillators. He discovered a phase transition from incoherent behavior with dispersed phases to synchrony with aligned frequencies and coherent (i.e., nearby) phases. Winfree found that this phase transition depends on the trade-off between the heterogeneity of the oscillator population and the strength of the mutual coupling, which he could formulate by parametric thresholds. However, Winfree's model was too general to be analytically tractable. Inspired by these works, Kuramoto (1975) simplified Winfree's model and arrived at the coupled oscillator dynamics (1) with a complete interaction graph and uniform weights $a_{i j}=K / n$ :

$$
\dot{\theta}_{i}=\omega_{i}-\frac{K}{n} \sum_{j=1}^{n} \sin \left(\theta_{i}-\theta_{j}\right), \quad i \in\{1, \ldots, n\} \text {. }
$$

In an ingenious analysis, Kuramoto (1975, 1984a) showed that synchronization occurs in the model (3) if 
the coupling gain $K$ exceeds a certain threshold $K_{\text {critical }}$ function of the distribution of the natural frequencies $\omega_{i}$. The dynamics (3) are nowadays known as the Kuramoto model of coupled oscillators, and Kuramoto's original work initiated a broad stream of research. A compelling historical perspective is offered by Strogatz (2000). We also recommend the surveys by Acebrón et al. (2005), Dorogovtsev et al. (2008), and Arenas et al. (2008).

Canonical model and prototypical example: Diffusively-coupled phase oscillators appear to be quite specific at first glance, but they are locally canonical models for weakly coupled and periodic limit-cycle oscillators (Hoppensteadt and Izhikevich, 1997). This fact is established in work by the computational neuroscience community which has developed different approaches (Ermentrout and Kopell, 1984, 1991; Hoppensteadt and Izhikevich, 1997; Izhikevich and Kuramoto, 2006; Izhikevich, 2007) to reduce general periodic limit-cycle oscillators and weak interaction models to diffusivelycoupled phase oscillator networks of the form

$$
\dot{\theta}_{i}=\omega_{i}+\sum_{j=1}^{n} h_{i j}\left(\theta_{i}-\theta_{j}\right)
$$

where $h_{i j}: \mathbb{S}^{1} \rightarrow \mathbb{R}$ are $2 \pi$-periodic coupling functions. Among such phase oscillators networks, the often encountered and most thoroughly studied case is that of anti-symmetric coupling without higher-order harmonics, that is, the oscillator network (1) with sinusoidal coupling. Moreover, the coupled oscillator model (1) serves as the prototypical example for synchronization in complex networks (Strogatz, 2001; Boccaletti et al., 2006; Osipov et al., 2007; Suykens and Osipov, 2008; Arenas et al., 2008), and its linearization is the well-known consensus protocol studied in networked control, see the surveys and monographs (Olfati-Saber et al., 2007; Ren et al., 2007; Bullo et al., 2009; Garin and Schenato, 2010; Mesbahi and Egerstedt, 2010). Indeed, numerous control scientists explored the coupled oscillator model (1) as a nonlinear generalization of the consensus protocol (Jadbabaie et al., 2004; Moreau, 2005; Scardovi et al., 2007; Olfati-Saber, 2006; Lin et al., 2007; Chopra and Spong, 2009; Sarlette and Sepulchre, 2009; Sepulchre, 2011).

The importance of phase oscillator networks does not stem only from their importance as local canonical models. Often they are naturally encountered in applications by first-principle modeling, as phenomenological models, or as a result of control design. In the following, we review a set of selected applications in sciences and technology.

Applications in sciences: The coupled oscillator model (1) and its generalization (4) appear in the study of biological synchronization and rhythmic phenomena. Example systems include pacemaker cells in the heart (Michaels et al., 1987), circadian cells in the brain (Liu et al., 1997), coupled cortical neurons (Crook et al., 1997), Hodgkin-Huxley neurons (Brown et al., 2003), brain networks (Varela et al., 2001), yeast cells (Ghosh et al., 1971), flashing fireflies (Buck, 1988; Ermentrout, 1991), chirping crickets (Walker, 1969), central pattern generators for animal locomotion (Kopell and Ermentrout, 1988), particle models mimicking animal flocking behavior (Ha et al., 2010b, 2011), and fish schools (Paley et al., 2007), among others. The coupled oscillator model (1) also appears in physics and chemistry in modeling and analysis of spin glass models (Daido, 1992; Jongen et al., 2001), flavor evolution of neutrinos (Pantaleone, 1998), coupled Josephson junctions (Wiesenfeld et al., 1998), coupled metronomes (Pantaleone, 2002), Huygen's coupled pendulum clocks (Bennett et al., 2002; Kapitaniak et al., 2012), micromechanical oscillators with optical (Zhang et al., 2012) or mechanical (Shim et al., 2007) coupling, and in the analysis of chemical oscillations (Kuramoto, 1984a; Kiss et al., 2002). Finally, oscillator networks of the form (1) also serve as phenomenological models for synchronization phenomena in social networks, such as rhythmic applause (Néda et al., 2000), opinion dynamics (Pluchino et al., 2006a,b), pedestrian crowd synchrony on London's Millennium bridge (Strogatz et al., 2005), and decision making in animal groups (Leonard et al., 2012).

Applications in engineering: Technological applications of the coupled oscillator model (1) and its generalization (4) include deep brain stimulation (Tass, 2003; Nabi and Moehlis, 2011; Franci et al., 2012), locking in solid-state circuit oscillators (Abidi and Chua, 1979; Mirzaei et al., 2007), planar vehicle coordination (Paley et al., 2007; Sepulchre et al., 2007, 2008; Klein, 2008; Klein et al., 2008), carrier synchronization without phase-locked loops (Rahman et al., 2011), synchronization in semiconductor laser arrays (Kozyreff et al., 2000), and microwave oscillator arrays (York and Compton, 2002). Since alternating current (AC) circuits are naturally modeled by equations similar to (1), some electric applications are found in structure-preserving (Bergen and Hill, 1981; Sauer and Pai, 1998) and networkreduced power system models (Chiang et al., 1995; Dörfler and Bullo, 2012b), and droop-controlled inverters in microgrids (Simpson-Porco et al., 2013). Algorithmic applications of the coupled oscillator model (1) include limit-cycle estimation through particle filters (Tilton et al., 2012), clock synchronization in decentralized computing networks (Simeone et al., 2008; Baldoni et al., 2010; Wang et al., 2013), central pattern generators for robotic locomotion (Aoi and Tsuchiya, 2005; Righetti and Ijspeert, 2006; Ijspeert, 2008), decentralized maximum likelihood estimation (Barbarossa and Scutari, 2007), and human-robot interaction (Mizumoto et al., 2010). Further envisioned applications of oscillator networks obeying equations similar to (1) include generating music (Huepe et al., 2012), signal processing (Shim et al., 2007), pattern recognition (Vassilieva et al., 2011), and neuro-computing through micromechanical (Hoppensteadt and Izhikevich, 2001) or laser (Hoppensteadt and Izhikevich, 2000; Wang and Ghosh, 
2007) oscillators.

Theoretical investigations: Coupled oscillator models of the form (1) are studied from a purely theoretical perspective in the physics, dynamical systems, and control communities. At the heart of the coupled oscillator dynamics is the transition from incoherence to synchrony. In this article we will be particularly interested in the notion of frequency synchronization, that is, in the property of certain solutions to reach equal frequencies $\dot{\theta}_{i}(t)$ among all oscillators. For infinitely many oscillators this notion can be relaxed to a subset of oscillators reaching frequency synchronization. We will also study conditions under which the angles $\theta_{i}(t)$ themselves synchronize, or they are tightly clustered (in a single or multiple groups), or they are spread evenly in regular patterns over the circle. We refer to the surveys and tutorials (Kuramoto, 1984b; Strogatz, 2000, 2001; Acebrón et al., 2005; Boccaletti et al., 2006; Arenas et al., 2008; Dorogovtsev et al., 2008; Dörfler and Bullo, 2011; Sarlette and Sepulchre, 2011; Mauroy et al., 2012; Francis, 2015) for an incomplete set of recent theoretic research activities. We will review and attribute relevant theoretical results throughout the course of this article.

\subsection{Contributions and Contents:}

This paper surveys the literature on synchronization in networks of coupled oscillators from a unified controltheoretical perspective. We present some selected applications relevant to control systems, we discuss a sample of important analysis methods based on controltheoretical concepts, and we provide a comprehensive review of the most-recent and sharpest results available for complex oscillator networks. For the sake of a clear and streamlined presentation, we present some selected applications, analysis methods, and results in detail, and only list the corresponding references otherwise. Due to the limited space, we can review only a selected subset of the expansive literature on this subject.

In Section 2, we review some selected technological applications of the coupled oscillator model (1) which are relevant to control systems. We present in some detail various problems in vehicle coordination, electric power networks, and clock synchronization, and we justify the importance of the phase oscillator networks (4) as canonical models of weakly coupled limit-cycle oscillators.

Prompted by these applications, Section 3 introduces the reader to different synchronization notions, including frequency and phase synchronization, phase balancing, pattern formation, and partial synchronization. These notions are defined for finite and infinite oscillator populations, connected through complete or sparsely-coupled networks. We illustrate these concepts with a simple yet rich example that nicely explains the basic phenomenology in coupled oscillator networks.
Section 4 presents a few basic results and useful analysis methods, including studies on the Jacobian linearization of the dynamics (1), appropriate Lyapunov functions, and (incremental) boundedness. These basic results will be exploited throughout the rest of the paper.

Section 5 surveys a set of important results for networks of identical oscillators. In particular, we cover phase synchronization, phase balancing, and pattern formation. We highlight contraction properties and potential function arguments as powerful analysis methods.

Section 6 is devoted to complete and uniformly-weighted networks of heterogeneous oscillators, that is, the classic Kuramoto model (3). We cover both finite-dimensional as well as infinite-dimensional populations and present a set of necessary, sufficient, implicit, and explicit conditions on the critical coupling strength $K_{\text {critical }}$. In this effort, we collect contributions from several references and arrive at novel results within a unified perspective.

Section 7 surveys synchronization metrics, results, and analysis methods for sparse networks of heterogeneous oscillators. We present two sufficient conditions for synchronization. The first condition comes with an estimate of the region of attraction, whereas the second condition is sharper but the regions of attraction of the synchronized solution is unknown in this case. Since both conditions are conservative for general network topologies and parameters, we also present a recent analysis approach leading to a sharp sufficient condition for certain classes of oscillator networks.

In the final Section 8 , we summarize the limitations of existing analysis methods and highlight some important directions for future research.

\subsection{Preliminaries and Notation:}

The remainder of this section recalls some standard notation and preliminaries from algebraic graph theory.

Vectors and functions: Let $\mathbb{1}_{n}$ and $\mathbb{O}_{n}$ be the $n$ dimensional vectors of unit and zero entries, and let $\mathbb{1}_{n}^{\perp}$ be the orthogonal complement of $\mathbb{1}_{n}$ in $\mathbb{R}^{n}$, that is, $\mathbb{1}_{n}^{\perp} \triangleq\left\{x \in \mathbb{R}^{n} \mid x \perp \mathbb{1}_{n}\right\}$. Accordingly, let $\mathbb{1}_{n \times n}$ denote the $(n \times n)$-dimensional matrix with unit entries. Given an $n$-tuple $\left(x_{1}, \ldots, x_{n}\right)$, let $x \in \mathbb{R}^{n}$ be the associated vector with maximum and minimum elements $x_{\max }$ and $x_{\min }$. Given an ordered index set $\mathcal{I}$ of cardinality $|\mathcal{I}|$ and a one-dimensional array $\left\{x_{i}\right\}_{i \in \mathcal{I}}$, let $\operatorname{diag}\left(\left\{x_{i}\right\}_{i \in \mathcal{I}}\right) \in \mathbb{R}^{|\mathcal{I}| \times|\mathcal{I}|}$ be the associated diagonal matrix. For $x \in \mathbb{R}^{n}$ define $\sin (x)=\left(\sin \left(x_{1}\right), \ldots, \sin \left(x_{n}\right)\right)$ and for $x \in[-1,1]^{n}$ define $\arcsin (x)=\left(\arcsin \left(x_{1}\right), \ldots, \arcsin \left(x_{n}\right)\right)$. Finally, define the continuous function sinc $: \mathbb{R} \rightarrow \mathbb{R}$ by $\operatorname{sinc}(x)=\sin (x) / x$, where $\operatorname{sinc}(0)=1$. 
Geometry on the $n$-torus: The set $\mathbb{S}^{1}$ denotes the unit circle, an angle is a point $\theta \in \mathbb{S}^{1}$, and an arc is a connected subset of $\mathbb{S}^{1}$. The $n$-torus is the Cartesian product $\mathbb{T}^{n}=\mathbb{S}^{1} \times \cdots \times \mathbb{S}^{1}$. The geodesic distance between two angles $\theta_{1}, \theta_{2}$ is the minimum of the counter-clockwise and the clockwise arc lengths connecting $\theta_{1}$ and $\theta_{2}$. With slight abuse of notation, let $\left|\theta_{1}-\theta_{2}\right|$ denote the geodesic distance between the two angles $\theta_{1}, \theta_{2} \in \mathbb{S}^{1}$.

Algebraic graph theory: Let $G(\mathcal{V}, \mathcal{E}, A)$ be an undirected, connected, and weighted graph without selfloops. Let $A \in \mathbb{R}^{n \times n}$ be its symmetric nonnegative adjacency matrix with zero diagonal elements $a_{i i}=0$. For each node $i \in\{1, \ldots, n\}$, define the node degree by $\operatorname{deg}_{i}=\sum_{j=1}^{n} a_{i j}$. Define the Laplacian matrix by $L=\operatorname{diag}\left(\left\{\operatorname{deg}_{i}\right\}_{i=1}^{n}\right)-A \in \mathbb{R}^{n \times n}$. If a unique number $\ell \in\{1, \ldots,|\mathcal{E}|\}$ and an arbitrary direction are assigned to each edge $\{i, j\} \in \mathcal{E}$, the (oriented) incidence matrix $B \in \mathbb{R}^{n \times|\mathcal{E}|}$ is defined component-wise by $B_{k \ell}=1$ if node $k$ is the sink node of edge $\ell$ and by $B_{k \ell}=-1$ if node $k$ is the source node of edge $\ell$; all other elements are zero. For $x \in \mathbb{R}^{n}$, the vector $B^{T} x \in \mathbb{R}^{|\mathcal{E}|}$ has entries of the form $x_{i}-x_{j}$ corresponding to the oriented edge from $j$ to $i$, that is, $B^{T}$ maps node variables $x_{i}, x_{j}$ to incremental edge variables $x_{i}-x_{j}$. If $\operatorname{diag}\left(\left\{a_{i j}\right\}_{\{i, j\} \in \mathcal{E}}\right)$ is the diagonal matrix of edge weights, then one can

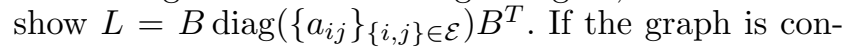
nected, then $\operatorname{Ker}\left(B^{T}\right)=\operatorname{Ker}(L)=\operatorname{span}\left(\mathbb{1}_{n}\right)$, all $n-1$ non-zero eigenvalues of $L$ are strictly positive, and the second-smallest eigenvalue $\lambda_{2}(L)$ is called the algebraic connectivity and is a spectral connectivity measure.

Since the Laplacian $L$ is singular, we will use its MoorePenrose pseudo inverse $L^{\dagger}$. If $V \in \mathbb{R}^{n \times n}$ is an orthonormal matrix of eigenvectors of $L$, the singular value decomposition of $L$ is $L=V \operatorname{diag}\left(0,\left\{\lambda_{i}\right\}_{i \in\{2, \ldots, n\}}\right) V^{T}$, and its Moore-Penrose pseudo inverse $L^{\dagger}$ is given by $L^{\dagger}=$ $V \operatorname{diag}\left(0,\left\{1 / \lambda_{i}\right\}_{i \in\{2, \ldots, n\}}\right) V^{T}$. A direct consequence of the singular value decomposition is the identity $L \cdot L^{\dagger}=$ $L^{\dagger} \cdot L=I_{n}-\frac{1}{n} \mathbb{1}_{n \times n}$. We also define the effective resistance between any two nodes $i$ and $j$ by $R_{i j}=L_{i i}^{\dagger}+$ $L_{j j}^{\dagger}-2 L_{i j}^{\dagger}$. We refer to (Dörfler and Bullo, 2013a) for further information and identities on Laplacian inverses and on the resistance distance.

Laplacian flow: A well-studied cost function associated with a graph is the Laplacian potential $1 / 2 \cdot x^{T} L x=1 / 2$. $\sum_{i, j=1}^{n} a_{i j}\left(x_{i}-x_{j}\right)^{2}$, defined for $x \in \mathbb{R}^{n}$. The associated gradient flow $\dot{x}=-L x$ is known as the Laplacian flow or consensus protocol, and it reads in components as

$$
\dot{x}_{i}=-\sum_{j=1}^{n} a_{i j}\left(x_{i}-x_{j}\right) .
$$

The consensus protocol is well-studied in the control literature (Olfati-Saber et al., 2007; Ren et al., 2007; Bullo et al., 2009; Garin and Schenato, 2010; Mesbahi and
Egerstedt, 2010), and it can be regarded as linear counterpart to the coupled oscillator model (1) with dynamics evolving on the Euclidean state space $\mathbb{R}^{n}$ and without drift terms. Some of the analysis tools and insights developed for the consensus protocol extend to the coupled oscillator model (1).

\section{Selected Examples of Oscillator Networks Relevant to Control Systems}

The mechanical analog in Fig. 1 provides an intuitive illustration of the coupled oscillator dynamics (1), and Section 1 contains a survey of a wide range of applications. Here, we detail some selected exemplary applications, which have recently received significant attention by the control community, and we justify the importance of the oscillator network (4) as a local canonical model.

\subsection{Flocking, Schooling, and Vehicle Coordination}

A recent research field in control is the coordination of autonomous vehicles based on locally available information and inspired by biological flocking phenomena. Consider a set of $n$ particles in the plane $\mathbb{R}^{2}$, which we identify with the complex plane $\mathbb{C}$. Each particle $i \in \mathcal{V}=$ $\{1, \ldots, n\}$ is characterized by its position $r_{i} \in \mathbb{C}$, its heading angle $\theta_{i} \in \mathbb{S}^{1}$, and a steering control law $u_{i}(r, \theta)$ depending on the position and heading of itself and other vehicles, see Fig. 2.(a). For simplicity, we assume that all particles have unit speed. The particle kinematics are then given by (Justh and Krishnaprasad, 2004)

$$
\begin{aligned}
& \dot{r}_{i}=e^{\mathrm{i} \theta_{i}}, \\
& \dot{\theta}_{i}=u_{i}(r, \theta),
\end{aligned}
$$

for $i \in\{1, \ldots, n\}$ and $\mathrm{i}=\sqrt{-1}$. If no control is applied, then particle $i$ travels in a straight line with orientation $\theta_{i}(0)$, and if $u_{i}=\omega_{i} \in \mathbb{R}$ is a nonzero constant, then particle $i$ traverses a circle with radius $1 /\left|\omega_{i}\right|$.

The interaction among the particles is modeled by a possibly time-varying interaction graph $G(\mathcal{V}, \mathcal{E}(t), A(t))$ determined by communication and sensing patterns. As shown by Vicsek et al. (1995), interesting motion patterns emerge if the controllers use only relative phase information between neighboring particles, that is, $u_{i}=$ $\omega_{0}(t)+f_{i}\left(\theta_{i}-\theta_{j}\right)$ for $\{i, j\} \in \mathcal{E}(t)$ and $\omega_{0}: \mathbb{R}_{\geq 0} \rightarrow$ $\mathbb{R}$. For example, the steering control $u_{i}=\omega_{0}(t)-K$. $\sum_{j=1}^{n} a_{i j}(t) \sin \left(\theta_{i}-\theta_{j}\right)$ with gain $K \in \mathbb{R}$ results in

$$
\dot{\theta}_{i}=\omega_{0}(t)-K \cdot \sum_{j=1}^{n} a_{i j}(t) \sin \left(\theta_{i}-\theta_{j}\right), \quad i \in \mathcal{V} .
$$

The controlled phase dynamics (7) correspond to the coupled oscillator model (1) with a time-varying interaction graph with weights $K \cdot a_{i j}(t)$ and identically timevarying natural frequencies $\omega_{i}=\omega_{0}(t)$. The controlled 

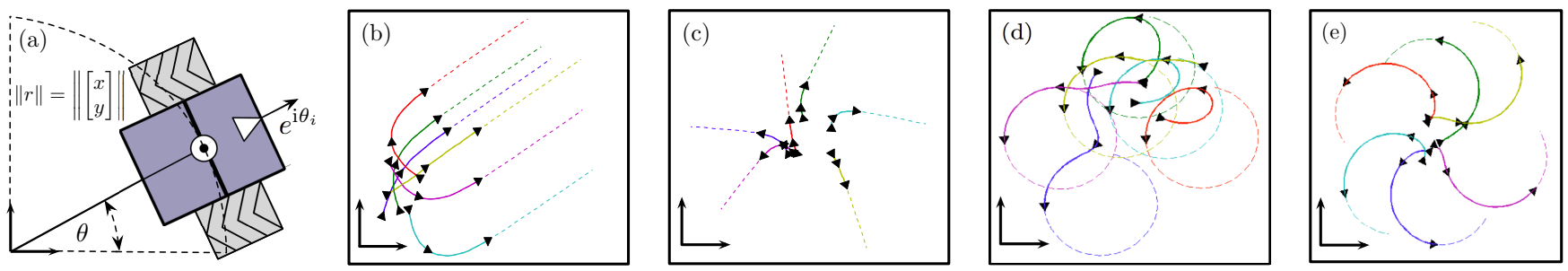

Fig. 2. Panel (a) illustrates the particle kinematics (6). Panels (b)-(e) illustrate the controlled dynamics (6)-(7) with $n=6$ particles, a complete interaction graph, and identical and constant natural frequencies: $\omega_{0}(t)=0$ in panels (b) and (c) and $\omega_{0}(t)=1$ in panels (d) and (e). The values of $K$ are $K=1$ in panel (b) and (d) and $K=-1$ in panel (c) and (e). The arrows depict the orientation, the dashed curves show the long-term position dynamics, and the solid curves show the initial transient position dynamics. As illustrated, the resulting motion displays "synchronized" or "balanced" heading angles for $K= \pm 1$, and translational motion for $\omega_{0}(t)=0$, respectively circular motion for $\omega_{0}(t)=1$.

phase dynamics (7) give rise to elegant and useful coordination patterns that mimic animal flocking behavior (Leonard et al., 2012) and fish schools (Paley et al., 2007). A few representative trajectories are illustrated in Fig. 2. Inspired by these biological phenomena, scientists have studied the controlled phase dynamics (7) and their variations in the context of tracking and formation controllers in swarms of autonomous vehicles. We refer to (Paley et al., 2007; Sepulchre et al., 2007, 2008; Klein, 2008; Klein et al., 2008; Scardovi, 2010; Leonard et al., 2012) for other control laws, motion patterns, and their analysis.

In the following sections, we will present various tools to analyze the motion patterns in Fig. 2, which we will refer to as phase synchronization (Fig. 2.(b) and Fig. 2.(d)) and phase balancing (Fig. 2.(c) and Fig. 2.(e)).

\subsection{Electric Power Networks with Synchronous Gener- ators and $D C / A C$ Inverters}

Consider an AC power network modeled as an undirected, connected, and weighted graph with $n$ nodes $\mathcal{V}=\{1, \ldots, n\}$, transmission lines $\mathcal{E} \subset \mathcal{V} \times \mathcal{V}$, and admittance matrix $Y=Y^{T} \in \mathbb{C}^{n \times n}$. For each node, consider the voltage phasor $V_{i}=\left|V_{i}\right| e^{\mathrm{i} \theta_{i}}$ corresponding to the phase $\theta_{i} \in \mathbb{S}^{1}$ and magnitude $\left|V_{i}\right| \geq 0$ of the sinusoidal solution to the circuit equations. If the network is lossless, then the active power flow from node $i$ to $j$ is $a_{i j} \sin \left(\theta_{i}-\theta_{j}\right)$, where we adopt the shorthand $a_{i j}=\left|V_{i}\right| \cdot\left|V_{j}\right| \cdot \Im\left(Y_{i j}\right)$, see Fig. 3.(a). The node set is partitioned as $\mathcal{V}=\mathcal{V}_{1} \cup \mathcal{V}_{2} \cup \mathcal{V}_{3}$, where $\mathcal{V}_{1}$ are load buses, $\mathcal{V}_{2}$ are synchronous generators, and $\mathcal{V}_{3}$ are grid-connected direct current (DC) power sources.

The active power drawn by a load $i \in \mathcal{V}_{1}$ consists of a constant term $P_{1, i}>0$ and a frequency-dependent term $D_{i} \dot{\theta}_{i}$ with $D_{i}>0$, see Fig. 3.(b). The resulting power balance equation is

$$
D_{i} \dot{\theta}_{i}+P_{1, i}=-\sum_{j=1}^{n} a_{i j} \sin \left(\theta_{i}-\theta_{j}\right), \quad i \in \mathcal{V}_{1}
$$

If the generator reactances are absorbed into the admittance matrix, then the electromechanical swing dynamics of the synchronous generator $i \in \mathcal{V}_{2}$ are

$$
M_{i} \ddot{\theta}_{i}+D_{i} \dot{\theta}_{i}=P_{\mathrm{m}, i}-\sum_{j=1}^{n} a_{i j} \sin \left(\theta_{i}-\theta_{j}\right), \quad i \in \mathcal{V}_{2}
$$

where $\theta_{i} \in \mathbb{S}^{1}$ and $\dot{\theta}_{i} \in \mathbb{R}^{1}$ are the generator rotor angle and frequency, $P_{\mathrm{m}, i}>0$ is the mechanical power input, and $M_{i}>0$, and $D_{i}>0$ are the inertia and damping coefficients. The dynamics (8)-(9) constitute the structurepreserving power network model, proposed by Bergen and Hill (1981). A derivation from first principles can be found in (Sauer and Pai, 1998, Chapter 7).

We assume that each DC source is connected to the $\mathrm{AC}$ grid via a $\mathrm{DC} / \mathrm{AC}$ inverter, the inverter output impedances are absorbed into the admittance matrix, and each inverter is equipped with a conventional droop controller. For a droop-controlled inverter $i \in \mathcal{V}_{3}$ with droop-slope $1 / D_{i}>0$, the deviation of the power output $\sum_{j=1}^{n} a_{i j} \sin \left(\theta_{i}-\theta_{j}\right)$ from its nominal value $P_{\mathrm{d}, i}>0$ is proportional to the frequency deviation $D_{i} \dot{\theta}_{i}$. This gives rise to the droop-controlled inverter dynamics (Simpson-Porco et al., 2013)

$$
D_{i} \dot{\theta}_{i}=P_{\mathrm{d}, i}-\sum_{j=1}^{n} a_{i j} \sin \left(\theta_{i}-\theta_{j}\right), \quad i \in \mathcal{V}_{3}
$$

These power network devices are illustrated as circuit elements in panels (a)-(d) of Fig. 3. Panels (e) and (f) show a high-voltage transmission network and a microgrid. We remark that different loads such as constant power/current/susceptance loads and synchronous motors can be modeled by the same set of equations (8)(10), see (Sastry and Varaiya, 1980; Chiang et al., 1995; Sauer and Pai, 1998; Dörfler and Bullo, 2013a).

Synchronization is pervasive in the operation of power networks. All generating units of an interconnected grid must remain in strict frequency synchronism while continuously following demand and rejecting disturbances. Notice that, with the exception of the inertial 
(a)

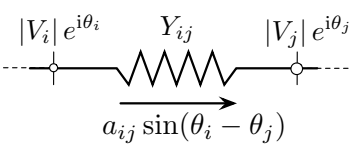

(c)

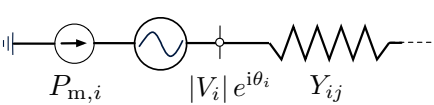

(b)

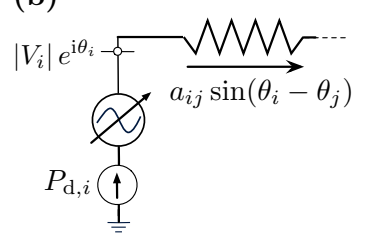

(d)

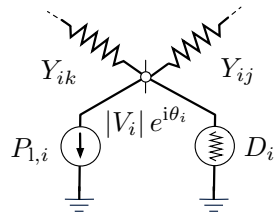

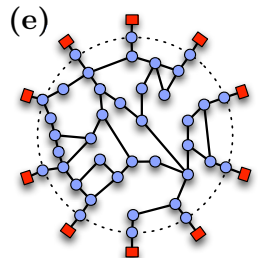

Fig. 3. Illustration of the power network devices as circuit elements. Subfigure (a) shows a transmission element connecting nodes $i$ and $j$. Subfigure (b) shows an inverter controlled according to (10). Subfigure (c) shows a synchronous generator. Subfigure (d) shows a frequency-dependent load. Subfigure (e) shows a schematic illustration of the IEEE 39 power grid, where the (red) squares depict synchronous generators and the (blue) circles are load buses. Finally, Subfigure (f) shows a microgrid based on the IEEE 37 feeder, where the (yellow) diamonds depict inverters and (black) circles are passive junctions.

terms $M_{i} \ddot{\theta}_{i}$ and the possibly non-unit coefficients $D_{i}$, the power network dynamics (8)-(10) are a perfect electrical analog of the coupled oscillator model (1) with $\omega_{i} \in\left\{-P_{1, i}, P_{\mathrm{m}, i}, P_{\mathrm{d}, i}\right\}$. Thus, it is not surprising that scientists from different disciplines recently advocated coupled oscillator approaches to analyze synchronization in power networks (Tanaka et al., 1997; Subbarao et al., 2001; Hill and Chen, 2006; Filatrella et al., 2008; Buzna et al., 2009; Fioriti et al., 2009; Simpson-Porco et al., 2013; Dörfler and Bullo, 2012b; Rohden et al., 2012; Dörfler et al., 2013; Mangesius et al., 2012; Motter et al., 2013; Ainsworth and Grijalva, 2013). The theoretical tools presented in this article establish how frequency synchronization in power networks depend on the nodal parameters $\left(P_{1, i}, P_{\mathrm{m}, i}, P_{\mathrm{d}, i}\right)$ as well as the interconnecting electrical network with weights $a_{i j}$.

\subsection{Clock Synchronization in Decentralized Networks}

Another emerging technological application of oscillator networks is clock synchronization in decentralized computing networks, such as wireless and distributed software networks. A natural approach to clock synchronization is to treat each clock as an oscillator and follow a diffusion-based (or pulse-coupling) protocol to synchronize them, see the surveys (Lindsey et al., 1985; Simeone et al., 2008) and the interesting recent results (Hong and Scaglione, 2005; Baldoni et al., 2010; Mallada and Tang, 2011; Wang et al., 2013; Wang and Doyle, 2012).

For illustration, consider a set of distributed processors $\mathcal{V}=\{1, \ldots, n\}$ connected by a (possibly directed) communication network. Each processor is equipped with an internal clock. These clocks need to be synchronized for distributed computing and network routing tasks. As discussed in the surveys by Lindsey et al. (1985) and Simeone et al. (2008), we consider only analog clocks with continuous coupling since digital clocks are essentially discretized analog clocks, and pulse-coupled clocks can be modeled continuously after a phase reduction and averaging analysis. For our purposes, the clock of processor $i$ is a voltage-controlled oscillator (VCO) generating a harmonic waveform $s_{i}(t)=\sin \left(\theta_{i}(t)\right)$, where $\theta_{i}(t)$ is the accumulated instantaneous phase. For uncoupled clocks, each phase $\theta_{i}(t)$ evolves according to

$\theta_{i}(t)=\left(\theta_{i}(0)+\frac{2 \pi}{T_{\mathrm{nom}}+T_{i}} t\right) \bmod (2 \pi), i \in\{1, \ldots, n\}$

where $T_{\text {nom }}>0$ is the nominal period, $T_{i} \in \mathbb{R}$ is an offset (or skew), and $\theta_{i}(0) \in \mathbb{S}^{1}$ is the initial phase. To synchronize their internal clocks, the processors follow a diffusion-based protocol. In a first step, neighboring oscillators communicate their respective waveforms $s_{i}(t)$ to another. Second, through a phase detector (PD) each node measures a convex combination of phase differences

$$
\operatorname{cvx}_{i}(\theta(t))=\sum_{j=1}^{n} a_{i j} f\left(\theta_{i}(t)-\theta_{j}(t)\right), i \in\{1, \ldots, n\}
$$

where $f: \mathbb{S}^{1} \rightarrow \mathbb{R}$ is an odd $2 \pi$-periodic function, and $a_{i j} \geq 0$ are detector-specific convex weights satisfying $\sum_{j=1}^{n} a_{i j}=1$. Finally, $\operatorname{cvx}_{i}(\theta(t))$ is fed to a phase-locked loop filter (PLL) whose output drives the local phase. A first-order and constant PLL with gain $K$ results in

$$
\dot{\theta}_{i}(t)=\frac{2 \pi}{T_{\mathrm{nom}}+T_{i}}+K \cdot \operatorname{cvx}_{i}(\theta(t)), \quad i \in\{1, \ldots, n\} .
$$

The diffusion-based synchronization protocol (11) is illustrated in Fig. 4, and its objective is to synchronize the frequencies $\dot{\theta}_{i}(t)$ and possibly also the phases $\theta_{i}(t)$ in the processor network. For an undirected communication protocol with symmetric weights $a_{i j}=a_{j i}$ and a sinusoidal coupling function $f(\cdot)=\sin (\cdot)$, the protocol (11) reduces to the coupled oscillator model (1).

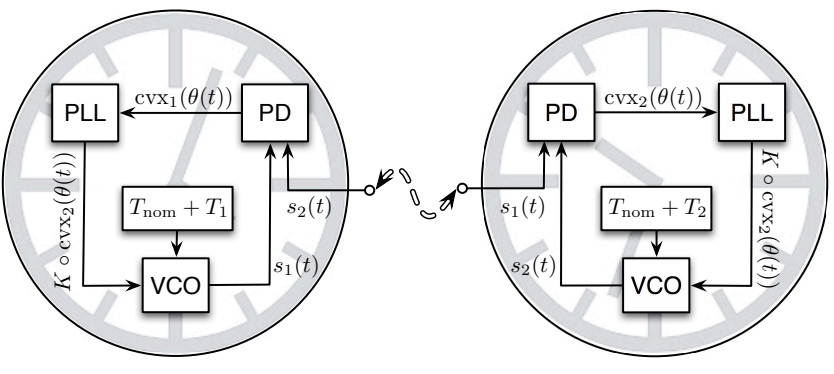

Fig. 4. Schematic illustration of the diffusion-based synchronization protocol (11) for two coupled analog clocks. 
The tools developed in the next section will enable us to state conditions when the protocol (11) successfully achieves phase or frequency synchronization. The protocol (11) is merely a starting point, more sophisticated higher-order PLLs can be constructed to enhance steady-state deviations from phase synchrony, communication and phase noise as well as non-constant timedelays can be considered in the design, and the phase coupling functions $f$ can be optimized to increase the synchronization rate or minimize energy consumption.

\subsection{Canonical Coupled Oscillator Model}

In the preceding subsections we have seen how the coupled-oscillator model (1) appears naturally in various applications. At first glance, diffusively-coupled phase oscillator models of the general form (4) appear to be quite specific. We now illustrate how the general phase oscillator network model (4) (and its particular instance (1)) can be obtained as a local canonical model of weakly coupled and periodic limit-cycle oscillators (Hoppensteadt and Izhikevich, 1997). Our presentation is informal, we schematically follow the approaches developed in the computational neuroscience community, and we refer to the textbooks (Hoppensteadt and Izhikevich, 1997; Izhikevich, 2007), the tutorials (Izhikevich and Kuramoto, 2006; Mauroy et al., 2012; Sacré and Sepulchre, 2014), and the pioneering papers (Winfree, 1967; Ermentrout and Kopell, 1984, 1991) for details.

Consider a limit-cycle oscillator modeled as a dynamical system with state $x \in \mathbb{R}^{m}$ and nonlinear dynamics $\dot{x}=f(x)$. Assume that this system admits a locally exponentially stable periodic orbit $\mathcal{S} \subset \mathbb{R}^{m}$ with period $T>0$. By a local change of variables, any trajectory in a neighborhood of $\mathcal{S}$ can be characterized by a phase variable $\varphi \in \mathbb{S}^{1}$ with dynamics $\dot{\varphi}=\Omega$, where $\Omega=2 \pi / T$. Now consider a weakly-forced oscillator of the form

$$
\dot{x}(t)=f(x(t))+\varepsilon g(t),
$$

where $\varepsilon>0$ is sufficiently small and $g(t)$ is a timedependent external forcing term. For sufficiently small forcing $\varepsilon g(t)$, the attractive limit cycle $\mathcal{S}$ persists, and the local phase dynamics are obtained as

$$
\dot{\varphi}(t)=\Omega+\varepsilon Q(\varphi(t)) g(t),
$$

where $\varphi \mapsto Q(\varphi)$ is the infinitesimal phase response curve (iPRC) and we dropped higher order terms $\mathcal{O}\left(\varepsilon^{2}\right)$. The iPRC is a linear response function that associates to each point on the periodic orbit $\mathcal{S}$ (parameterized by the phase $\varphi$ ) the phase shift induced by the input $\varepsilon g(t)$.

Now consider $n$ such limit-cycle oscillators. Let $x_{i} \in \mathbb{R}^{m}$ be the state of oscillator $i$ with limit cycle $\mathcal{S}_{i} \subset \mathbb{R}^{m}$ and period $T_{i}>0$. We assume that the oscillators are weakly coupled with interconnected dynamics given by

$$
\dot{x}_{i}=f_{i}\left(x_{i}\right)+\varepsilon \sum_{j=1}^{n} g_{i j}\left(x_{i}, x_{j}\right), \quad i \in\{1, \ldots, n\}
$$

Here, $g_{i j}$ is the coupling function for the oscillators $i, j \in$ $\{1, \ldots, n\}$. The coupling functions may be continuous or impulsive or take the value zero if oscillators $i$ and $j$ are not interacting. The weak coupling in (13) can be identified with the weak forcing in (12), and a transformation to phase coordinates results in the local phase model

$$
\dot{\varphi}_{i}=\Omega_{i}+\varepsilon \sum_{j=1}^{n} Q_{i}\left(\varphi_{i}\right) g_{i j}\left(x_{i}\left(\varphi_{i}\right), x_{j}\left(\varphi_{j}\right)\right)
$$

where $\Omega_{i}=2 \pi / T_{i}$. By changing variables as $\theta_{i}(t)=$ $\varphi_{i}(t)-\Omega_{i} t$, we arrive at the coupled phase dynamics

$\dot{\theta}_{i}=\varepsilon \sum_{j=1}^{n} Q_{i}\left(\theta_{i}+\Omega_{i} t\right) g_{i j}\left(x_{i}\left(\theta_{i}+\Omega_{i} t\right), x_{j}\left(\theta_{j}+\Omega_{j} t\right)\right)$.

An averaging analysis applied to the $\theta$-dynamics yields

$$
\dot{\theta}_{i}=\varepsilon \omega_{i}+\varepsilon \sum_{j=1}^{n} h_{i j}\left(\theta_{i}-\theta_{j}\right),
$$

where the averaged coupling functions $h_{i j}$ are given by

$h_{i j}(\chi)=\lim _{T \rightarrow \infty} \frac{1}{T} \int_{0}^{T} Q_{i}\left(\Omega_{i} \tau\right) g_{i j}\left(x_{i}\left(\Omega_{i} \tau\right), x_{j}\left(\Omega_{j} \tau-\chi\right)\right) d \tau$,

and $\omega_{i}=h_{i i}(0)$. Notice that the averaged coupling functions $h_{i j}$ are $2 \pi$-periodic and the coupling in (14) is diffusive. In the slow time scale $\tau=\varepsilon t$, the averaged dynamics (14) equal the phase oscillator network (4). This analysis justifies calling the phase oscillator network (4) a local canonical model for weakly coupled limit-cycle oscillators. It also explains the widespread adoption of phase oscillator models as phenomenological model in synchronization studies. If the interaction among the oscillators is anti-symmetric, then all functions $h_{i j}$ are odd, and a first-order Fourier series expansion yields $h_{i j}(\cdot) \approx a_{i j} \sin (\cdot)$ as first harmonic with coefficient $a_{i j}$. In this case, the dynamics (14) in the slow time scale $\tau=\varepsilon t$ reduce to the coupled oscillator model (1).

As a prototypical example, for two van der Pol oscillators (with parameters in the quasi-harmonic limit) coupled through a resistor, the above procedure results exactly in the coupled oscillator model (1), see (Rand and Holmes, 1980; Mauroy et al., 2012). In general, the coupling functions $h_{i j}$ depend on the iPRC and may not be sinusoidal. Hence, the iPRC serves as a natural analysis (Sacré and Sepulchre, 2014; Sacré, 2013; Brown et al., 2004) and design (Wang et al., 2013; Wang and Doyle, 2012) tool for general limit-cycle oscillator networks. 
(a)

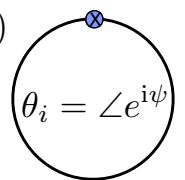

(b)

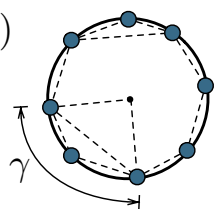

(c)

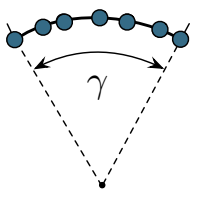

(d)

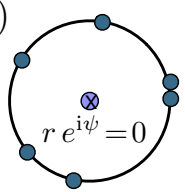

(e)

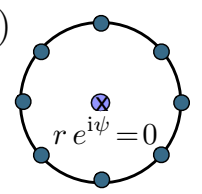

Fig. 5. Different phase configurations exhibited by frequency-synchronized solutions of the oscillator network (1): (a) phase synchronization, (b) phase cohesiveness, (c) arc invariance, (d) phase balancing, and (e) splay state synchronization.

\section{Synchronization Notions and Metrics}

In this section, we introduce different notions of synchronization illustrated in Fig. 5. We first address various commonly-studied notions of synchronization associated with coherent behavior and cohesive phases. We then address the concept of phase balancing and splay states. Finally, we also discuss the setting of infinitedimensional systems.

\subsection{Synchronization Notions}

The coupled oscillator model (1) evolves on $\mathbb{T}^{n}$ and features an important symmetry, namely, the rotational invariance of the angular variable $\theta$. This symmetry gives rise to the structure of the state space and the different synchronization properties that the model (1) can display. We first review the case of a finite oscillator population, where all notions of synchronized solutions share the common property that the frequencies are equal to a constant synchronization frequency.

Frequency synchronization: A solution $\theta: \mathbb{R}_{\geq 0} \rightarrow$ $\mathbb{T}^{n}$ achieves frequency synchronization if all frequencies $\dot{\theta}_{i}(t)$ converge to a common constant frequency $\omega_{\text {sync }} \in$ $\mathbb{R}$ as $t \rightarrow \infty$. The explicit synchronization frequency $\omega_{\text {sync }} \in \mathbb{R}$ of the coupled oscillator model (1) can be obtained by summing over all equations in (1) as $\sum_{i=1}^{n} \dot{\theta}_{i}=$ $\sum_{i=1}^{n} \omega_{i}$. In the frequency-synchronized case, this sum simplifies to $\sum_{i=1}^{n} \omega_{\mathrm{sync}}=\sum_{i=1}^{n} \omega_{i}$. In conclusion, if a solution of the coupled oscillator model (1) achieves frequency synchronization, then it does so with synchronization frequency equal to $\omega_{\text {sync }}=\sum_{i=1}^{n} \omega_{i} / n$. By transforming to a rotating frame with frequency $\omega_{\text {sync }}$ and by replacing $\omega_{i}$ with $\omega_{i}-\omega_{\text {sync }}$, we obtain $\omega_{\text {sync }}=0$ (or, equivalently, $\omega \in \mathbb{1}_{n}^{\perp}$ ). In what follows, without loss of generality, we assume that $\omega \in \mathbb{1}_{n}^{\perp}$ so that $\omega_{\text {sync }}=0$.

Phase synchronization: A solution $\theta: \mathbb{R}_{>0} \rightarrow \mathbb{T}^{n}$ to the coupled oscillator model (1) achieves phase synchronization if all phases $\theta_{i}(t)$ become identical as $t \rightarrow \infty$.

Remark 1 (Terminology) In the vast synchronization literature, alternative terminologies for phase synchronization include full, exact, or perfect synchronization. For a frequency-synchronized solution all phase distances $\left|\theta_{i}(t)-\theta_{j}(t)\right|$ are constant, and the terminology phase locking is sometimes used instead of frequency synchronization. Other commonly used terms instead of frequency synchronization include frequency locking, frequency entrainment, or also partial synchronization.

Phase cohesiveness: As we will see later, phase synchronization can occur only if all natural frequencies $\omega_{i}$ are identical. If the natural frequencies are not identical, then each pairwise distance $\left|\theta_{i}(t)-\theta_{j}(t)\right|$ can converge to a constant but not necessarily zero value. The concept of phase cohesiveness formalizes this possibility. For $\gamma \in\left[0, \pi\left[\right.\right.$, let $\bar{\Delta}_{G}(\gamma) \subset \mathbb{T}^{n}$ be the closed set of angle arrays $\left(\theta_{1}, \ldots, \theta_{n}\right)$ with the property $\left|\theta_{i}-\theta_{j}\right| \leq \gamma$ for all $\{i, j\} \in \mathcal{E}$, that is, each pairwise phase distance is upper bounded by $\gamma$. Also, let $\Delta_{G}(\gamma)$ be the interior of $\bar{\Delta}_{G}(\gamma)$. A solution $\theta: \mathbb{R}_{\geq 0} \rightarrow \mathbb{T}^{n}$ is then said to be phase cohesive if there exists a length $\gamma \in[0, \pi[$ such that $\theta(t) \in \bar{\Delta}_{G}(\gamma)$ for all $t \geq 0$. Notice that a phase cohesive solution is also phase synchronous when $\gamma=0$.

The main object under study in most applications and theoretic analyses are phase-cohesive and frequencysynchronized solutions, where all oscillators rotate with the same frequency and all the pairwise phase distances are upper bounded. In the following, we restrict our attention to synchronized solutions with sufficiently small phase distances $\left|\theta_{i}-\theta_{j}\right| \leq \gamma<\pi / 2$ for $\{i, j\} \in \mathcal{E}$. Of course, there may exist other synchronized solutions with larger phase distances, but these are not necessarily stable (see our analysis in Section 4) and/or not relevant in most applications. ${ }^{2}$ In what follows, in the interest of brevity, we call a solution synchronized if it is frequency synchronized and phase cohesive.

Synchronization manifold: A geometric object of interest is the synchronization manifold. Given a point $r \in \mathbb{S}^{1}$ and an angle $s \in[0,2 \pi]$, let $\operatorname{rot}_{s}(r) \in \mathbb{S}^{1}$ be the rotation of $r$ counterclockwise by the angle $s$. For $\left(r_{1}, \ldots, r_{n}\right) \in \mathbb{T}^{n}$, define the equivalence class

$\left[\left(r_{1}, \ldots, r_{n}\right)\right]=\left\{\left(\operatorname{rot}_{s}\left(r_{1}\right), \ldots, \operatorname{rot}_{s}\left(r_{n}\right)\right) \in \mathbb{T}^{n} \mid s \in[0,2 \pi]\right\}$.

Clearly, if $\left(r_{1}, \ldots, r_{n}\right) \in \bar{\Delta}_{G}(\gamma)$ for some $\gamma \in[0, \pi / 2[$, then $\left[\left(r_{1}, \ldots, r_{n}\right)\right] \subset \bar{\Delta}_{G}(\gamma)$. Given a synchronized so-

\footnotetext{
${ }^{2}$ For example, in power network applications the coupling terms $a_{i j} \sin \left(\theta_{i}-\theta_{j}\right)$ are power flows along transmission lines $\{i, j\} \in \mathcal{E}$, and the phase distances $\left|\theta_{i}-\theta_{j}\right|$ are bounded well below $\pi / 2$ due to thermal constraints. In Subsection 3.4, we present a converse synchronization notion termed phase balancing, where the goal is to maximize phase distances.
} 
lution characterized by $\theta_{\text {sync }} \in \bar{\Delta}_{G}(\gamma)$ for some $\gamma \in$ $\left[0, \pi / 2\left[\right.\right.$, the set $\left[\theta_{\text {sync }}\right] \subset \bar{\Delta}_{G}(\gamma)$ is a synchronization manifold of the coupled-oscillator model (1). Note that a synchronized solution takes value in a synchronization manifold due to rotational symmetry, and for $\omega \in$ $\mathbb{1}_{n}^{\perp}$ (implying $\omega_{\text {sync }}=0$ ) a synchronization manifold is also an equilibrium manifold of the coupled oscillator model (1). These geometric concepts are illustrated in Fig. 6 for the two-dimensional case.

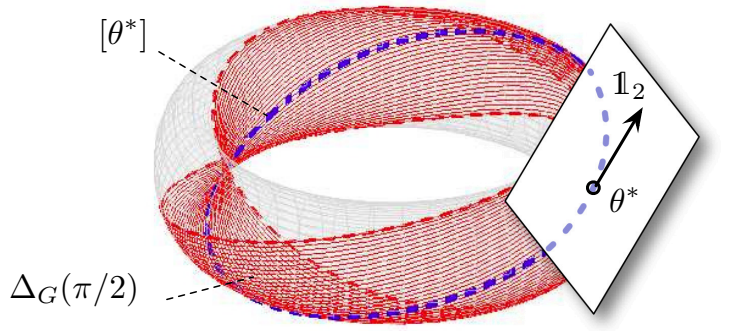

Fig. 6. Illustration of the state space $\mathbb{T}^{2}$, the set $\Delta_{G}(\pi / 2)$, the synchronization manifold $\left[\theta^{*}\right]$ associated to a phase-synchronized angle array $\theta^{*}=\left(\theta_{1}^{*}, \theta_{2}^{*}\right) \in \bar{\Delta}_{G}(0)$, and the tangent space with translation vector $\mathbb{1}_{2}$ at $\theta^{*}$.

Arc invariance: To conclude our list of synchronization notions, we introduce the concept of arc invariance. For $\gamma \in\left[0,2 \pi\left[\right.\right.$, let $\overline{\operatorname{Arc}}_{n}(\gamma) \subset \mathbb{T}^{n}$ be the closed set of angle arrays $\theta=\left(\theta_{1}, \ldots, \theta_{n}\right)$ with the property that there exists an arc of length $\gamma$ containing all $\theta_{1}, \ldots, \theta_{n}$. Thus, an angle array $\theta \in \overline{\operatorname{Arc}}_{n}(\gamma)$ satisfies $\max _{i, j \in\{1, \ldots, n\}} \mid \theta_{i}-$ $\theta_{j} \mid \leq \gamma$. Finally, let $\operatorname{Arc}_{n}(\gamma)$ be the interior of the set $\overline{\operatorname{Arc}}_{n}(\gamma)$. A solution $\theta: \mathbb{R}_{\geq 0} \rightarrow \mathbb{T}^{n}$ is then said to be arc invariant if there exists a length $\gamma \in[0,2 \pi[$ such that $\theta(t) \in \overline{\operatorname{Arc}}_{n}(\gamma)$ for all $t \geq 0$. Notice that $\overline{\operatorname{Arc}}_{n}(\gamma) \subseteq \bar{\Delta}_{G}(\gamma)$ but the two sets are generally not equal. For a complete coupling graph, sufficiently many oscillators, and for sufficiently small $\gamma$, the two sets become equal, and arc invariance is an appropriate synchronization notion, see, e.g., Theorems 5.2 and 6.6.

\subsection{A Simple yet Illustrative Example}

The following example illustrates different notions of synchronization and points out various important geometric subtleties occurring on the compact state space $\mathbb{T}^{2}$. Consider $n=2$ oscillators with $\omega_{2} \geq 0 \geq \omega_{1}=-\omega_{2}$. We restrict our attention to angles contained in $\operatorname{Arc}_{n}(\pi)$ : for angles $\theta_{1}, \theta_{2}$ with $\left|\theta_{2}-\theta_{1}\right|<\pi$, the angular difference $\theta_{2}-\theta_{1}$ is the number in ] $-\pi, \pi$ [ with magnitude equal to the geodesic distance $\left|\theta_{2}-\theta_{1}\right|$ and with positive sign if and only if the counter-clockwise path length from $\theta_{1}$ to $\theta_{2}$ is smaller than the clockwise path length. With this definition the two-dimensional oscillator dynamics $\left(\dot{\theta}_{1}, \dot{\theta}_{2}\right)$ can be reduced to the scalar difference dynamics $\dot{\theta}_{2}-\dot{\theta}_{1}$. After scaling time as $t \mapsto t\left(\omega_{2}-\omega_{1}\right)$ and intro- ducing $\kappa=2 a_{12} /\left(\omega_{2}-\omega_{1}\right)$, the difference dynamics are

$$
\frac{d}{d t}\left(\theta_{2}-\theta_{1}\right)=f_{\kappa}\left(\theta_{2}-\theta_{1}\right) \triangleq 1-\kappa \sin \left(\theta_{2}-\theta_{1}\right) .
$$

The one-parameter family of dynamical systems (15) can be analyzed graphically by plotting the scalar vector field $f_{\kappa}\left(\theta_{2}-\theta_{1}\right)$, for $\theta_{2}-\theta_{1} \in[0, \pi]$; see Fig. $7(\mathrm{a})$. The vector field features a saddle-node bifurcation at $\kappa=1$. For $\kappa<1$ no equilibria exist. For $\kappa>1$ we have an asymptotically stable equilibrium $\left.\theta_{\text {stable }}=\arcsin \left(\kappa^{-1}\right) \in\right] 0, \pi / 2[$ and an unstable equilibrium $\theta_{\text {unstable }}=\arcsin \left(\kappa^{-1}\right) \in$ ]$\pi / 2, \pi\left[\right.$. For $\kappa>1$ and $\left(\theta_{2}(0)-\theta_{1}(0)\right) \in\left[0, \theta_{\text {unstable }}[\right.$, all trajectories converge to $\theta_{\text {stable, that is, the oscilla- }}$ tors synchronize and remain phase cohesive (or arc invariant). For $\left(\theta_{2}(0)-\theta_{1}(0)\right) \notin\left[0, \theta_{\text {unstable }}\right.$ the difference $\theta_{2}(t)-\theta_{1}(t)$ increases beyond $\pi$, and $\theta_{2}(t)-\theta_{1}(t)$ converges asymptotically to the equilibrium $\theta_{\text {stable }}$ in the set where $\theta_{2}-\theta_{1}<0$. Equivalently, in the configuration space $\mathbb{S}^{1}$ the oscillators revolve once around the circle before converging to $\left[\theta_{\text {stable }}\right]$. Since $\sin \left(\theta_{\text {stable }}\right)=$ $\sin \left(\theta_{\text {unstable }}\right)=\kappa^{-1}$, in the limit $\kappa \rightarrow \infty$ the oscillators achieve phase synchronization from every initial condition in an open semi-circle $\operatorname{Arc}_{2}(\pi)$. In the critical case, $\kappa=1$, the saddle equilibrium manifold at [ $\left.\theta_{\text {saddle }}\right]$ is globally attractive but not stable, see Fig. 7(b).

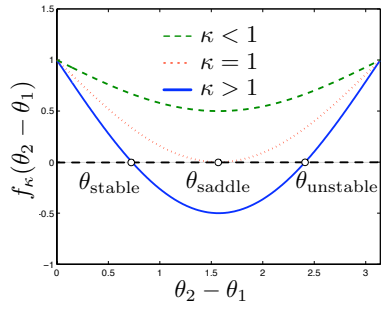

(a) Vector field in eq. (15)

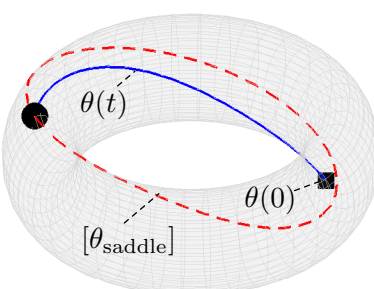

(b) Trajectory $\theta(t)$ for $\kappa=1$
Fig. 7. Plot of the vector field (15) for $\theta_{2}-\theta_{1}>0$ and various values of $\kappa$ and a trajectory $\theta(t) \in \mathbb{T}^{2}$ for the critical case $\kappa=1$, where the dashed line is the saddle equilibrium manifold and $\boldsymbol{\square}$ and $\bullet$ depict $\theta(0)$ and $\lim _{t \rightarrow \infty} \theta(t)$.

In conclusion, the simple but already rich 2-dimensional case shows that two oscillators are phase cohesive and synchronize if and only if $\kappa>1$, that is, if and only if the coupling dominates the heterogeneity as $2 a_{12}>\omega_{2}-\omega_{1}$. The ratio $1 / \kappa$ determines the asymptotic phase cohesiveness as well as the set of admissible initial conditions. More general oscillator networks display the same phenomenology, but the threshold from incoherence to synchrony is generally unknown. Finally, we remark that for oscillator networks of dimension $n \geq 3$, this loss of synchrony via a saddle-node bifurcation is only the starting point of a series of bifurcations occurring if the coupling is further decreased, see (Maistrenko et al., 2005; Tönjes, 2007; Popovych et al., 2005; Suykens and Osipov, 2008). 


\subsection{Synchronization Metrics}

The notions of phase cohesiveness and arc invariance are performance measures for synchronization, and phase synchronization can be characterized as the extreme case of phase cohesiveness with $\lim _{t \rightarrow \infty} \theta(t) \in \bar{\Delta}_{G}(0)=\overline{\operatorname{Arc}}_{n}(0)$. An alternative performance measure is the magnitude of the so-called order parameter introduced by Kuramoto $(1975,1984 \mathrm{a})$ as

$$
r e^{\mathrm{i} \psi}=\frac{1}{n} \sum_{j=1}^{n} e^{\mathrm{i} \theta_{j}}
$$

The order parameter (16) is the centroid of all oscillators represented as points on the unit circle in $\mathbb{C}^{1}$. The magnitude $r$ of the order parameter is a synchronization measure: if the oscillators are phase-synchronized, then $r=1$, and if the oscillators are spaced equally on the unit circle, then $r=0$, see Fig. 5(e). The latter case is characterized in detail in Subsection 3.4. Because the order parameter (16) is the centroid of the oscillators, it is contained within the convex hull of the smallest arc containing all oscillators, see the illustration in Fig. 8. Hence, the magnitude $r$ of the order parameter can be related to the arc length $\gamma$ as in the following lemma.

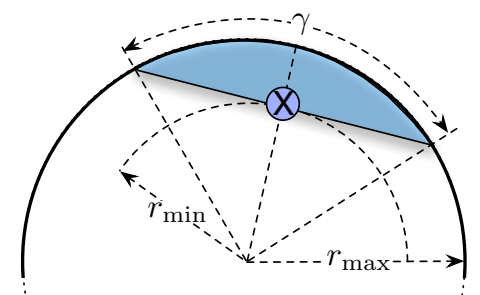

Fig. 8. Schematic illustration of an arc of length $\gamma \in[0, \pi]$, its convex hull (shaded), and the value $\otimes$ of the corresponding order parameter $r e^{\mathrm{i} \psi}$ with minimum magnitude $r_{\min }=\cos (\gamma / 2)$ and maximum magnitude $r_{\max }=1$.

Lemma 3.1 (Shortest arc length and order parameter) Given an angle array $\theta=\left(\theta_{1}, \ldots, \theta_{n}\right) \in \mathbb{T}^{n}$ with $n \geq 2$, let $r(\theta)=\frac{1}{n}\left|\sum_{j=1}^{n} e^{\mathrm{i} \theta_{j}}\right|$ be the magnitude of the order parameter, and let $\gamma(\theta)$ be the length of the shortest arc containing all angles, that is, $\theta \in \overline{\operatorname{Arc}}_{n}(\gamma(\theta))$. The following statements hold:

1) if $\gamma(\theta) \in[0, \pi]$, then $r(\theta) \in[\cos (\gamma(\theta) / 2), 1]$; and

2) if $\theta \in \overline{\operatorname{Arc}}_{n}(\pi)$, then $\gamma(\theta) \in[2 \arccos (r(\theta)), \pi]$.

For a complete graph, the asymptotic magnitude $r$ of the order parameter serves as an average performance index for synchronization, and arc invariance can be understood as a worst-case performance index. Appropriate definitions of the order parameter tailored to noncomplete graphs (and noisy dynamics) have been proposed by Jadbabaie et al. (2004); Restrepo et al. (2005); Scardovi et al. (2007); Paley et al. (2007); Sonnenschein and Schimansky-Geier (2012); Ichinomiya (2004).

\subsection{Phase Balancing, Splay State, and Patterns}

Applications in neuroscience (Crook et al., 1997; Varela et al., 2001; Brown et al., 2003), deep-brain stimulation (Tass, 2003; Nabi and Moehlis, 2011; Franci et al., 2012), vehicle coordination (Paley et al., 2007; Sepulchre et al., 2007, 2008; Klein, 2008; Klein et al., 2008), and central pattern generators for locomotion purposes (Ijspeert, 2008; Aoi and Tsuchiya, 2005; Righetti and Ijspeert, 2006) motivate the study of coherent behaviors with synchronized frequencies where the phases are not cohesive, but rather dispersed in appropriate patterns. Whereas the phase-synchronized state is characterized by the order parameter $r$ achieving its maximal (unit) magnitude, we say that a solution $\theta: \mathbb{R}_{\geq 0} \rightarrow \mathbb{T}^{n}$ to the coupled oscillator model (1) achieves phase balancing if all phases $\theta_{i}(t)$ asymptotically converge to the set

$$
\mathrm{Bal}_{n}=\left\{\theta \in \mathbb{T}^{n}|r(\theta)=| \sum_{j=1}^{n} e^{\mathrm{i} \theta_{j}} / n \mid=0\right\},
$$

that is, asymptotically the oscillators are uniformly distributed over the unit circle $\mathbb{S}^{1}$ so that their centroid converges to the origin; see Fig. 5(d) and 5(e) for illustrations. We refer to Sepulchre et al. (2007) for a geometric characterization of the balanced state. One balanced state of particular interest in neuroscience applications is the so-called splay state $\left\{\theta \in \mathbb{T}^{n} \mid \theta_{i}=i \cdot 2 \pi / n+\varphi\right.$ $\left.(\bmod 2 \pi), \varphi \in \mathbb{S}^{1}, i \in\{1, \ldots, n\}\right\} \subseteq \mathrm{Bal}_{n}$ corresponding to phases uniformly distributed around the unit circle $\mathbb{S}^{1}$ with distances $2 \pi / n$, see Fig. $5(\mathrm{e})$.

Other highly-symmetric balanced states consist of multiple clusters of collocated oscillators, where the clusters themselves are arranged in splay state. In particular, if $m$ is a divisor of $n$, we define a symmetric balanced $(m, n)$-pattern to be a symmetric arrangement of the $n$ phases consisting of $m$ clusters uniformly spaced on $\mathbb{S}^{1}$, where each cluster contains $n / m$ synchronized phases. Fig. 9 illustrates all symmetric balanced $(m, n)$-patterns for $n=12$. Notice that, for any $n \in \mathbb{N}$, there are at

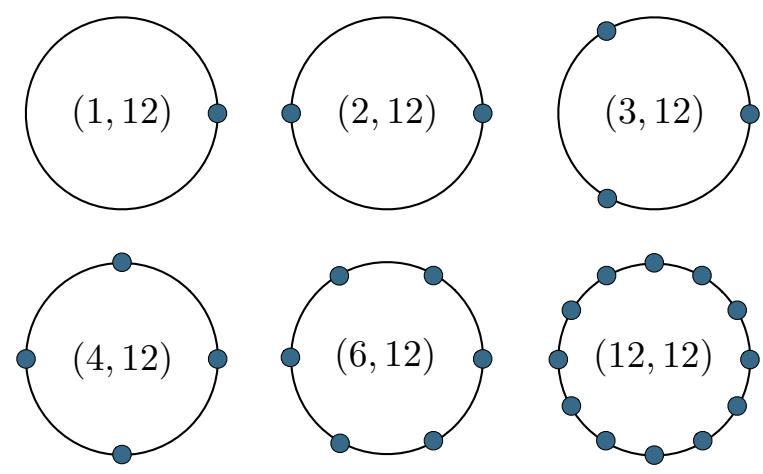

Fig. 9. Illustration of all symmetric balanced $(m, n)$-patterns for $n=12$. The $(1,12)$-pattern equals phase synchronization with $r=1$, all other patterns are phase-balanced configurations with $r=0$, and the $(12,12)$-pattern is the splay state. 
least two symmetric patterns: the $(1, n)$-pattern (i.e., the phase-synchronized state) and the $(n, n)$-pattern (i.e., the splay state). Arbitrary $(m, n)$-patterns can be stabilized, for example, by using coupling functions with higher harmonics, such as $\sin \left(m\left(\theta_{i}-\theta_{j}\right)\right)$; see (Sepulchre et al., 2007, 2008). The topic of symmetric phase balancing is similar in spirit to pattern formation (Cross and Hohenberg, 1993; Arcak, 2012), where phase synchronization corresponds to the "flat" solution with uniform phases and balanced $(m, n)$-patterns correspond to spatially non-uniform solutions with "higher-order modes."

\subsection{Synchronization in Infinite-Dimensional Networks}

For a complete coupling graph with uniform weights $a_{i j}=K / n$, where $K>0$, the coupled oscillator model (1) reduces to the celebrated Kuramoto model given in (3). By means of the order parameter $r e^{\mathrm{i} \psi}$ defined in equation (16), the Kuramoto model (3) can be rewritten in the insightful form

$$
\dot{\theta}_{i}=\omega_{i}-K r \sin \left(\theta_{i}-\psi\right), \quad i \in\{1, \ldots, n\}
$$

Equation (17) gives the intuition that the oscillators synchronize because of their coupling to a mean field represented by the order parameter $r e^{\mathrm{i} \psi}$, which itself is a function of $\theta(t)$. Intuitively, for small coupling strength $K$ each oscillator rotates with its distinct natural frequency $\omega_{i}$, whereas for large coupling strength $K$ all angles $\theta_{i}(t)$ will entrain to the mean field $r e^{\mathrm{i} \psi}$, and the oscillators synchronize. The transition from incoherence to synchronization occurs at a critical threshold value of the coupling strength, denoted by $K_{\text {critical }}$.

The analysis of this phase transition based on a meanfield and statistical mechanics viewpoint has been the subject of numerous investigations, starting with $\mathrm{Ku}$ ramoto's own ingenious analysis in (Kuramoto, 1975, 1984a). As neatly described by Strogatz (2000), Kuramoto assumed the a priori existence of solutions to (17) which feature a stationary order parameter $r(t) e^{\mathrm{i} \psi(t)}=$ constant. Following this assumption and his intuition, Kuramoto derived a set of self-consistency equations. A rigorous mathematical underpinning to Kuramoto's mean-field approach can be established by a time-scale separation (Ha and Slemrod, 2011) or in the continuum limit as the number of oscillators tends to infinity, and the natural frequencies $\omega$ are sampled from a distribution function $g: \mathbb{R} \rightarrow \mathbb{R}_{>0}$. The continuum-limit model has enjoyed a considerable amount of attention by the physics and dynamics communities. Related controltheoretical applications of the continuum-limit model are estimation of gait cycles (Tilton et al., 2012), spatial power grid modeling and analysis (Mangesius et al., 2012), and game theoretic approaches (Yin et al., 2012).

Continuum-limit model: Since infinite-dimensional oscillator networks are surveyed in detail in (Strogatz,
2000; Acebrón et al., 2005; Balmforth and Sassi, 2000), this paper discusses them only very briefly. In what follows, we present an informal Eulerian derivation of the continuum-limit model. We also remark that a treatment of (17) as a stochastic differential equation (in the limit of zero additive white noise) results in a FokkerPlanck equation analogous to the continuum-limit model (Crawford, 1994; Strogatz, 2000; Acebrón et al., 2005).

Consider an infinite population of oscillators, and let $\rho: \mathbb{S}^{1} \times \mathbb{R}_{\geq 0} \times \mathbb{R} \rightarrow \mathbb{R}_{\geq 0}$ be the probability density function of the oscillators, that is, $\int_{0}^{\gamma} \int_{\underline{\omega}}^{\bar{\omega}} \rho(\theta, t, \omega) g(\omega) d \omega d \theta$ denotes the fraction of oscillators in $\overline{\operatorname{Arc}}_{n}(\gamma) \subseteq \mathbb{S}^{1}$, at time $t$, and with frequencies $\omega \in[\underline{\omega}, \bar{\omega}]$. Hence, the order parameter is given by

$$
r(t) e^{\mathrm{i} \psi(t)}=\int_{0}^{2 \pi} \int_{-\infty}^{\infty} e^{\mathrm{i} \theta} \rho(\theta, t, \omega) g(\omega) d \omega d \theta .
$$

Notice that in the discrete (finite-dimensional) case we have $\rho(\theta, t, \omega)=\frac{1}{n} \sum_{j=1}^{n} \delta\left(\theta-\theta_{j}\right)$ (where $\delta(\cdot)$ is the Dirac $\delta$-distribution), and the two order parameters (16) and (18) coincide. According to (17), the instantaneous velocity of an oscillator at position $\theta$, at time $t$, and with natural frequency $\omega$ is given by $v(\theta, t, \omega)=\omega-$ $K r(t) \sin (\theta-\psi(t))$. The evolution of the probability density function is then governed by the continuity equation

$$
\frac{\partial}{\partial t} \rho+\frac{\partial}{\partial \theta}(\rho v)=0
$$

subject to the conservation of the oscillators at time $t$ and with frequency $\omega$, that is, $\int_{0}^{2 \pi} \rho(\theta, t, \omega) d \theta=1 .^{3}$

Synchronization in the continuum-limit model: Similar to the finite-dimensional model (16)-(17), the continuum-limit model (18)-(19) displays a rich set of symmetries (Ott and Antonsen, 2008) and dynamics (Balmforth and Sassi, 2000; Martens et al., 2009). The saddle-node bifurcation from incoherence to synchrony in the finite-dimensional model (16)-(17) (see Subsection 3.2) manifests itself in the infinite-dimensional model (18)-(19) as a phase transition from the uniform incoherent state with density $\rho(\theta, t, \omega)=\frac{1}{2 \pi}$ to the so-called partially-synchronized state. The partiallysynchronized state is characterized by a subset of phaselocked oscillators rotating in unison whereas the remaining oscillators are incoherent. ${ }^{4}$ The synchronized

\footnotetext{
3 In some articles, the continuum-limit model (18)(19) is presented with the density function $\tilde{\rho}(\theta, t, \omega)=$ $\rho(\theta, t, \omega) g(\omega)$, which satisfies $\int_{0}^{2 \pi} \tilde{\rho}(\theta, t, \omega) d \theta=g(\omega)$.

4 An analogous partially-synchronized state can be defined in the finite-dimensional Kuramoto model (16)-(17), where a subset of oscillators is contained in an arc; see (Aeyels and Rogge, 2004; De Smet and Aeyels, 2007). A related dynamic phenomenon are chimera states with frequency-synchronized and incoherent oscillators (Laing, 2009; Martens et al., 2013).
} 
set of oscillators are those satisfying $K r>|\omega|$ such that $v(\theta, t, \omega)=0$, and the incoherent ones are uniformly spread over the circle, see Fig. 10(a) and Fig. 10(b) for a schematic illustration. This phase transition occurs when $K$ exceeds some critical value $K_{\text {partial }}$. When $K$ is further increased, more and more oscillators become entrained by the mean field (18) and join the set of phase-locked oscillators. For a frequency distribution $g(\omega)$ with bounded support, there exists a second critical parameter $K_{\text {lock }} \geq K_{\text {partial }}$, such that for $K>K_{\text {lock }}$ all oscillators are phase-locked. This final stage of synchronization is illustrated in Fig. 10(c). It is often referred to as the fully phase-locked state, and it is reminiscent of frequency synchronization and arc invariance as displayed in the finite-dimensional model (16),(17).
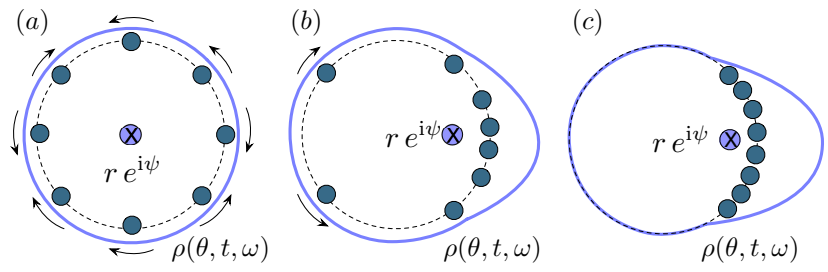

Fig. 10. Subfigure (a) displays the uniform incoherent state $\rho(\theta, t, \omega)=1 /(2 \pi)$. Subfigure (b) illustrates the partially-synchronized state, where a subset of oscillators rotates in unison and the remaining oscillators are incoherent. Subfigure (c) illustrates the fully phase-locked state.

Whereas the majority of the literature on the continuumlimit model (18)-(19) focuses on the first phase transition and the calculation of $K_{\text {partial }}$, see (Kuramoto, 1984a; Crawford, 1994; Strogatz, 2000; Acebrón et al., 2005; Mirollo and Strogatz, 2007; Balmforth and Sassi, 2000; Ott and Antonsen, 2008; Chiba, 2014) and references therein, the articles (Ermentrout, 1985; Hemmen and Wreszinski, 1993; Mirollo and Strogatz, 2005, 2007; Roberts, 2008; Verwoerd and Mason, 2011) discuss the fully phase-locked state and the calculation of $K_{\text {lock }}$. The influential works (Ott and Antonsen, 2008, 2009) exploit the extensive symmetries of the continuum-limit model (18)-(19) to construct a simple solution ansatz obeying low-dimensional ordinary differential equations. This reduction approach has triggered an extensive line of recent research, e.g., see (Martens et al., 2009; Laing, 2009; Pikovsky and Rosenblum, 2011; So et al., 2014) and references therein.

\section{Basic Analysis Methods and Results}

In this section, we review a few fundamental insights into the coupled oscillator dynamics (1), we state some key lemmas, and we introduce some analysis methods which will be exploited throughout the rest of the paper.

\subsection{Jacobian Analysis}

We begin by drawing some insights from a Jacobian analysis. The right-hand side of the oscillator network (1) defines the vector field $f: \mathbb{T}^{n} \rightarrow \mathbb{R}^{n}$ with components

$$
f_{i}(\theta)=\omega_{i}-\sum_{j=1}^{n} a_{i j} \sin \left(\theta_{i}-\theta_{j}\right), i \in\{1, \ldots, n\} .
$$

Because $\frac{\partial}{\partial \theta_{i}} f_{i}(\theta)=-\sum_{j=1}^{n} a_{i j} \cos \left(\theta_{i}-\theta_{j}\right)$ and $\frac{\partial}{\partial \theta_{j}} f_{i}(\theta)=a_{i j} \cos \left(\theta_{i}-\theta_{j}\right)$, the Jacobian $J(\theta)$ of the coupled oscillator model (1) satisfies

$$
J(\theta)=-B \operatorname{diag}\left(\left\{a_{i j} \cos \left(\theta_{i}-\theta_{j}\right)\right\}_{\{i, j\} \in \mathcal{E}}\right) B^{T},
$$

where $B$ is the incidence matrix of the graph. Notice that for phase cohesive angles $\theta \in \Delta_{G}(\pi / 2)$, the Jacobian $J(\theta)$ equals minus the Laplacian matrix of the graph $G(\mathcal{V}, \mathcal{E}, \tilde{A})$ with strictly positive weights $\tilde{a}_{i j}=$ $a_{i j} \cos \left(\theta_{i}-\theta_{j}\right)>0$, for $\{i, j\} \in \mathcal{E}$. Hence, $J(\theta)$ is negative semidefinite, it inherits the sparsity of the graph $G(\mathcal{V}, \mathcal{E}, A)$, and its nullspace $\operatorname{Span}\left(\mathbb{1}_{n}\right)$ arises from the rotational symmetry of the vector field (20). These basic results are fundamental to various analysis approaches. To the best of the authors' knowledge this set of results was first established by Tavora and Smith (1972b,a), and it has since been rediscovered several times. Some consequences are collected in the following lemma, whose proof can be found in (Dörfler et al., 2013, Lemma 3).

Lemma 4.1 (Stable synchronization in $\Delta_{G}(\pi / 2)$ ) Consider the coupled oscillator model (1) with a connected graph $G(\mathcal{V}, \mathcal{E}, A)$ and frequencies $\omega \in \mathbb{1}_{n}^{\perp}$. If there exists an equilibrium $\theta^{*} \in \Delta_{G}(\pi / 2)$, then

(i) $-J\left(\theta^{*}\right)$ is a negative semidefinite Laplacian matrix;

(ii) the equilibrium manifold $\left[\theta^{*}\right] \subset \Delta_{G}(\pi / 2)$ is locally exponentially stable (modulo rotational symmetry).

Some consequences of the particular form of the Jacobian (21) evaluated in $\Delta_{G}(\pi / 2)$ are collected below.

Frequency dynamics: The frequency dynamics obtained by differentiating the phase dynamics (1) are

$$
\frac{d}{d t} \dot{\theta}_{i}=-\sum_{j=1}^{n} \tilde{a}_{i j}(t)\left(\dot{\theta}_{i}-\dot{\theta}_{j}\right), \quad i \in\{1, \ldots, n\}
$$

where $\tilde{a}_{i j}(t)=a_{i j} \cos \left(\theta_{i}(t)-\theta_{j}(t)\right)$. The frequency dynamics (22) evolve on the tangent space of $\mathbb{T}^{n}$, that is, the Euclidean space $\mathbb{R}^{n}$. If the set $\bar{\Delta}_{G}(\gamma)$ is forward invariant and $\theta(0) \in \bar{\Delta}_{G}(\gamma)$ for some $\gamma \in[0, \pi / 2[$, then $a_{i j}(t) \geq a_{i j} \cos (\gamma)>0$, for $\{i, j\} \in \mathcal{E}$. Thus, the frequency dynamics (22) can be regarded as linear consensus protocol (5) with time-varying strictly-positive weights. Based on this observation, it can be shown that all frequencies $\dot{\theta}_{i}(t)$ synchronize exponentially, that is,

$$
\left\|\dot{\theta}(t)-\omega_{\mathrm{sync}} \mathbb{1}_{n}\right\|_{2} \leq\left\|\dot{\theta}(0)-\omega_{\mathrm{sync}} \mathbb{1}_{n}\right\|_{2} e^{\lambda_{\mathrm{fe}} t},
$$

where $\lambda_{\mathrm{fe}}=-\lambda_{2}(L) \cos (\gamma)$. We refer to (Chopra and Spong, 2009, Theorem 3.1) and (Dörfler and Bullo, 
$2012 \mathrm{~b}$, Theorem 4.1) for a formal proof, and to (Schmidt et al., 2012, Lemma 3.5), (Dörfler and Bullo, 2012b, Theorem 4.1), and (Wang and Doyle, 2013, Theorem 4) for extensions to more general coupling functions, time-delays, and extensions to models with pacemakers.

Contraction and incremental stability: Assume again that $\bar{\Delta}_{G}(\gamma)$ is forward invariant for some $\gamma \in[0, \pi / 2[$. Since $-J(\theta)$ is negative semidefinite in $\bar{\Delta}_{G}(\gamma)$, it follows that the coupled oscillator dynamics (1) are contracting ${ }^{5}$ relative to the nullspace $\mathbb{1}_{n}$. Consequently, the dynamics (1) are incrementally exponentially stable (modulo symmetry), that is, given any two initial values $\theta(0) \in \bar{\Delta}_{G}(\gamma)$ and $\tilde{\theta}(0) \in \bar{\Delta}_{G}(\gamma)$, there is a pseudo-metric $d: \mathbb{T}^{n} \times \mathbb{T}^{n} \rightarrow \mathbb{R}_{>0}$ (more precisely, a metric defined modulo symmetry) ${ }^{6}$ and constants $c_{1} \geq 1$ and $c_{2}>0$ such that

$$
d(\theta(t), \tilde{\theta}(t)) \leq c_{1} e^{-c_{2} t} d(\theta(0), \tilde{\theta}(0)), \quad \forall t \geq 0 .
$$

The application of contraction analysis to the coupled oscillator model (1) yields the incremental exponential stability (24) in $\ell_{2}$-type metrics (Chung and Slotine, 2010, Theorem 7 ) or in $\ell_{\infty}$-type metrics (Forni and Sepulchre, 2014, Example 6). Choi et al. (2011a, Theorem 4.1) report the incremental stability $(24)$ in an $\ell_{1}$-metric. Finally, for discontinuous and monotone coupling functions and complete interaction graphs the total variation distance provides yet another $\ell_{1}$-type contraction metric (Mauroy and Sepulchre, 2012).

Jacobian analysis beyond $\Delta_{G}(\pi / 2)$ : The results discussed so far in Subsection 4.1 are applicable only to angles inside the phase cohesive set $\Delta_{G}(\pi / 2)$, where all weights $\tilde{a}_{i j}=a_{i j} \cos \left(\theta_{i}-\theta_{j}\right)$ are strictly positive for $\{i, j\} \in \mathcal{E}$, and the Laplacian properties of the Jacobian $J(\theta)$ can be exploited. Outside the set $\Delta_{G}(\pi / 2)$, the associated state-dependent graph $G(\mathcal{V}, \mathcal{E}, \tilde{A})$ may at times be disconnected and/or have negative weights $\tilde{a}_{i j}=a_{i j} \cos \left(\theta_{i}-\theta_{j}\right)<0$. In this more general setting, the standard methods from algebraic and spectral graph theory cannot be applied and many puzzling examples are known (Araposthatis et al., 1981). A necessary condition for stability of arbitrary equilibrium manifolds $\left[\theta^{*}\right] \subset \mathbb{T}^{n}$ is that the graph induced by the Jacobian $J\left(\theta^{*}\right)$ possess a spanning tree with strictly positive weights $\tilde{a}_{i j}>0$ along its edges (Do et al., 2012). Sufficient stability and instability conditions can be derived if the graph induced by $J\left(\theta^{*}\right)$ admits certain cutsets

\footnotetext{
${ }^{5}$ We refer the reader to (Lohmiller and Slotine, 1998; Sontag, 2010) for a treatment of contraction analysis and to (Wang and Slotine, 2005; Russo et al., 2010; Forni and Sepulchre, 2014) for its extension to systems with symmetries.

6 The pseudo-metric $d$ is a nonnegative and symmetric function $\left(d\left(\theta_{1}, \theta_{2}\right)=d\left(\theta_{2}, \theta_{1}\right)\right)$ satisfying the triangle inequality $d\left(\theta_{1}, \theta_{2}\right) \leq d\left(\theta_{1}, \theta_{3}\right)+d\left(\theta_{3}, \theta_{2}\right)$ and $d\left(\theta_{1}, \theta_{1}\right)=0$ if and only if $\left[\theta_{1}\right]=\left[\theta_{2}\right]$. The pseudo-metric $d$ is a proper distance function on the quotient manifold $\mathbb{T}^{n} / \mathbb{S}^{1}$.
}

(Araposthatis et al., 1981; Bergen and Hill, 1981; Chandrashekhar and Hill, 1986; Mallada and Tang, 2014). Finally, for the complete graph with uniform weights (see the Kuramoto model (3)), additional insights and identities related to the Jacobian (21) can be found in (Aeyels and Rogge, 2004; Mirollo and Strogatz, 2005; Verwoerd and Mason, 2008; Bronski et al., 2012).

\subsection{Potential Landscape Analysis}

A classic analysis approach to oscillator networks with symmetric coupling can be deduced from the potential landscape. The potential energy $U: \mathbb{T}^{n} \rightarrow \mathbb{R}$ of the spring network in Fig. 1 is, up to an additive constant,

$$
U(\theta)=\sum_{\{i, j\} \in \mathcal{E}} a_{i j}\left(1-\cos \left(\theta_{i}-\theta_{j}\right)\right)
$$

For the complete graph with uniform weights $K / n$, the magnitude $r$ of the order parameter and the potential energy $U(\theta)$ are related by $\frac{2}{n} U(\theta)=1-r^{2}$. One can easily verify that the phase-synchronized state is a local minimum of the potential energy.

Given the potential energy in equation (25), the coupled oscillator model (1) can be reformulated as the forced gradient system

$$
\dot{\theta}_{i}=\omega_{i}-\frac{\partial}{\partial \theta_{i}} U(\theta), \quad i \in\{1, \ldots, n\}
$$

This rewriting clarifies the competition between the synchronization-enforcing coupling through $U(\theta)$ and the synchronization-inhibiting heterogeneous natural frequencies $\omega_{i}$. The unforced system (26) with $\omega=\mathbb{O}_{n}$ is a negative gradient flow $\dot{\theta}=-\partial U(\theta) / \partial \theta$ with the potential function $U(\theta)$ as natural Lyapunov function.

Since the Jacobian $J(\theta)$ is the negative Hessian of the potential $U(\theta)$, Lemma 4.1 implies that any equilibrium in $\Delta_{G}(\pi / 2)$ is a local minimizer of $U(\theta)$. Of particular interest are so-called $\mathbb{S}^{1}$-synchronizing graphs for which all critical points of (25) are hyperbolic, the phasesynchronized state is the global minimum of $U(\theta)$, and all other critical points are local maxima or saddle points. The class of $\mathbb{S}^{1}$-synchronizing graphs includes, among others, complete graphs and acyclic graphs (Monzón and Paganini, 2005; Canale and Monzón, 2008; Sarlette, 2009; Canale et al., 2010b,a). These basic results motivated the study of the critical points and of the curvature of the potential energy $U(\theta)$ in the literature on the theory and applications of synchronization, including, the study of transient stability in power systems and the design of motion coordination controllers for planar vehicles, see Subsections 2.1 and 2.2. Some direct consequences of the gradient formulation (26) and of the associated Hessian matrix (21) will be presented in Section 5 . 


\subsection{Absolute and Incremental Boundedness}

In this subsection we start from the basic observation that the sinusoidal interaction terms in equation (1) are upper bounded by the nodal degree $\operatorname{deg}_{i}=\sum_{j=1}^{n} a_{i j}$ of each oscillator. Hence, the natural frequencies $\left(\omega_{1}, \ldots, \omega_{n}\right)$ have to satisfy certain bounds, relative to the nodal degree, if a synchronized solution is to exist.

Lemma 4.2 (Necessary sync condition) Consider the coupled oscillator model (1) with graph $G(\mathcal{V}, \mathcal{E}, A)$, frequencies $\omega \in \mathbb{1}_{n}^{\perp}$, and nodal degree $\operatorname{deg}_{i}=\sum_{j=1}^{n} a_{i j}$ for each node $i \in\{1, \ldots, n\}$. If there exists a synchronized solution $\theta \in \bar{\Delta}_{G}(\gamma)$ for some $\gamma \in[0, \pi / 2]$, then the following conditions hold:

1) Absolute bound: For each node $i \in\{1, \ldots, n\}$,

$$
\operatorname{deg}_{i} \sin (\gamma) \geq\left|\omega_{i}\right|
$$

2) Incremental bound: For distinct $i, j \in\{1, \ldots, n\}$,

$$
\left(\operatorname{deg}_{i}+\operatorname{deg}_{j}\right) \sin (\gamma) \geq\left|\omega_{i}-\omega_{j}\right| \text {. }
$$

This lemma follows directly from the fact that synchronized solutions must satisfy $\dot{\theta}_{i}=0$ and $\dot{\theta}_{i}-\dot{\theta}_{j}=0$ for all $i, j \in\{1, \ldots, n\}$, see (Dörfler et al., 2013, Lemma 3) for a formal proof. Along the same lines, condition (27) can also be extended from a single node to a cutset in the graph (Ainsworth and Grijalva, 2013, Theorem 1).

Notice that the necessary conditions (27) and (28) are conservative estimates since they can be attained only if all angular distances $\left|\theta_{i}-\theta_{k}\right|$ and $\left|\theta_{j}-\theta_{k}\right|$ take the value $\gamma$, which is generally not possible. We will show in Lemma 6.4 below how to improve upon these necessary conditions in the case of a complete graph.

\section{Synchronization of Identical Oscillators}

In this section we present several analysis approaches and results for the study of synchronization in homogeneous oscillator networks, that is, oscillator models of the form (1) with identical natural frequencies.

\subsection{Phase Synchronization}

It can be easily verified that for non-zero and dissimilar natural frequencies $\omega \in \mathbb{1}_{n}^{\perp}$, the coupled oscillator model (1) does not admit a phase-synchronized solution of the form $\theta_{i}(t)=\theta_{j}(t)$ for all $i, j \in\{1, \ldots, n\}$. On the contrary, if all natural frequencies are identical, $\omega_{i} \equiv \omega_{0}$ for all $i \in\{1, \ldots, n\}$, then a transformation of the oscillator network (1) to a rotating frame with frequency $\omega_{0}$ yields

$$
\dot{\theta}_{i}=-\sum_{j=1}^{n} a_{i j} \sin \left(\theta_{i}-\theta_{j}\right), \quad i \in\{1, \ldots, n\}
$$

An elegant analysis of the coupled oscillator model (29) follows the insights developed in Subsections 4.1 and 4.2. System (29) is a negative gradient flow $\dot{\theta}=-\partial U(\theta) / \partial \theta$ defined by the smooth function $U(\theta)$ with compact sublevel sets. Hence, the LaSalle Invariance Principle (Khalil, 2002, Theorem 4.4) asserts that every solution converges to set of critical points $\left\{\theta \in \mathbb{T}^{n} \mid \partial U(\theta) / \partial \theta=\mathbb{O}_{n}\right\}$. This basic convergence result using potential functions and the LaSalle Invariance Principle has long been known in the neuroscience community, see, for example, (Cohen and Grossberg, 1983; Hoppensteadt and Izhikevich, 1997). Recent research efforts focus predominantly on establishing almost global synchronization. By Lemma 4.1, the phasesynchronized equilibrium manifold $[\theta] \in \bar{\Delta}_{G}(0)$ is locally exponentially stable, and for a $\mathbb{S}^{1}$-synchronizing graph, all other equilibria are unstable. We collect these observations in the following result presented, among others, in (Jadbabaie et al., 2004; Monzón and Paganini, 2005; Scardovi et al., 2007; Sepulchre et al., 2007).

Theorem 5.1 (Phase synchronization) Consider the coupled oscillator model (1) with a connected graph $G(\mathcal{V}, \mathcal{E}, A)$ and with natural frequencies $\omega \in \mathbb{R}^{n}$. The following statements are equivalent:

(i) Homogeneity: there exists a constant $\omega_{0} \in \mathbb{R}$ such that $\omega_{i}=\omega_{0}$ for all $i \in\{1, \ldots, n\}$; and

(ii) Local phase sync: there exists a locally exponentially stable phase synchronization manifold $\bar{\Delta}_{G}(0)$.

If the two equivalent cases (i) and (ii) are true, the following statements hold:

1) Global convergence: For all initial angles $\theta(0) \in$ $\mathbb{T}^{n}$, the frequencies $\dot{\theta}(t)$ converge to $\omega_{0} \mathbb{1}_{n}$ and the phases $\theta(t)$ converge to $\left\{\theta \in \mathbb{T}^{n} \mid \partial U(\theta) / \partial \theta=\mathbb{O}_{n}\right\}$; and

2) Almost global stability: If $G(\mathcal{V}, \mathcal{E}, A)$ is $\mathbb{S}^{1}$ synchronizing, the region of attraction of the phase synchronization manifold $\bar{\Delta}_{G}(0)$ is almost all of $\mathbb{T}^{n}$.

A representative simulation is shown in Fig. 12(a) below. The corresponding discrete-time analog to Theorem 5.1 can be found in (Klein, 2008; Klein et al., 2008; Scardovi et al., 2007). If higher order models with dynamic coupling are considered, then almost globally stable phase synchronization can be achieved for arbitrary connected (and also directed) graphs; see (Scardovi et al., 2007; Sepulchre et al., 2008; Lunze, 2011) for details.

\subsection{Consensus, Contraction, \& Convexity}

The interest of the control community in oscillator networks (1) was initially sparked by Jadbabaie et al. (2004) and Moreau (2005), who analyzed networks of identical oscillators as nonlinear extensions of the consensus protocol (5). Indeed, for zero natural frequencies $\omega=\mathbb{O}_{n}$ and 
for angles contained in an open semicircle $\theta \in \operatorname{Arc}_{n}(\pi)$, the dynamics (29) can be projected onto the real line via the local coordinate map $\varphi:]-\pi / 2, \pi / 2\left[{ }^{n} \rightarrow \mathbb{R}^{n}\right.$ defined by $x_{i}=\varphi_{i}\left(\theta_{i}\right)=\tan \left(\theta_{i}\right)$. With this projection proposed by Moreau (2005), the dynamics (29) are rewritten as the consensus-type model

$$
\dot{x}_{i}=-\sum_{j=1}^{n} b_{i j}(x)\left(x_{i}-x_{j}\right),
$$

where $b_{i j}(x)=a_{i j} \sqrt{\left(1+x_{i}^{2}\right) /\left(1+x_{j}^{2}\right)} \geq 0$. In particular, for $\theta \in \overline{\operatorname{Arc}}_{n}(\gamma)$ for some $\gamma \in\left[0, \pi\left[\right.\right.$, we have that $b_{i j}(x) \geq$ $a_{i j} / \sec (\gamma / 2)>0$ is strictly positive for all $\{i, j\} \in \overline{\mathcal{E}}$. A similar viewpoint is taken by Jadbabaie et al. (2004), where the coupled oscillator model (29) is rewritten as

$$
\dot{\theta}_{i}=-\sum_{j=1}^{n} c_{i j}(\theta)\left(\theta_{i}-\theta_{j}\right)
$$

where $c_{i j}(\theta)=a_{i j} \operatorname{sinc}\left(\theta_{i}-\theta_{j}\right) \geq 0$. Again, we have that $c_{i j}(\theta) \geq a_{i j} \operatorname{sinc}(\gamma)>0$ for $\{i, j\} \in \mathcal{E}$ and $\theta \in \overline{\operatorname{Arc}}_{n}(\gamma)$, $\gamma \in[0, \pi[$. Further consensus-theoretical derivations of the oscillator network (29) can be found in (Olfati-Saber, 2006; Sarlette and Sepulchre, 2009; Sepulchre, 2011).

In both formulations (30) and (31), the dynamics (29) are regarded as a consensus protocol (5) with strictly positive weights whose values are time-varying or statedependent. This interpretation is well defined provided that $\theta(t) \in \overline{\operatorname{Arc}}_{n}(\gamma)$ for all $t \geq 0$ and for some $\gamma \in[0, \pi[$. Different Lyapunov functions can be used to assure this boundedness, for example, the potential function $U(\theta)$ or standard quadratic Lyapunov functions used in consensus theory. Generally, the level sets of these Lyapunov functions are hard to characterize and provide poor estimates on the region of attraction. Another natural Lyapunov function is simply the length of the shortest arc containing all oscillators. This approach relies upon the contraction property, it has been developed for general nonlinear consensus systems, and it aims at showing that the convex hull of all states is decreasing, e.g., see (Moreau, 2004, 2005; Lin et al., 2007; Sepulchre, 2011).

Recall the geodesic distance on $\mathbb{S}^{1}$ and define the continuous function $V: \mathbb{T}^{n} \rightarrow[0, \pi]$ by

$$
V(\psi)=\max \left\{\left|\psi_{i}-\psi_{j}\right| \mid i, j \in\{1, \ldots, n\}\right\} .
$$

If all angles at time $t$ are contained in an arc of length strictly less than $\pi$, then the arc length $V(\theta(t))=$ $\max _{i, j \in\{1, \ldots, n\}}\left|\theta_{i}(t)-\theta_{j}(t)\right|$ is a Lyapunov function candidate for phase synchronization, see Fig. 11. Intuitively, the oscillators $\theta_{\ell}(t)$ and $\theta_{r}(t)$ at both boundaries are pulled towards their neighbors in the interior $\operatorname{Arc}_{n}(V(\theta(t))$, and the Lyapunov function $V(\theta(t))$ is non-increasing. The technical analysis is slightly more subtle since the function $V(\theta(t))$ is continuous but not necessarily differentiable when the maximum geodesic

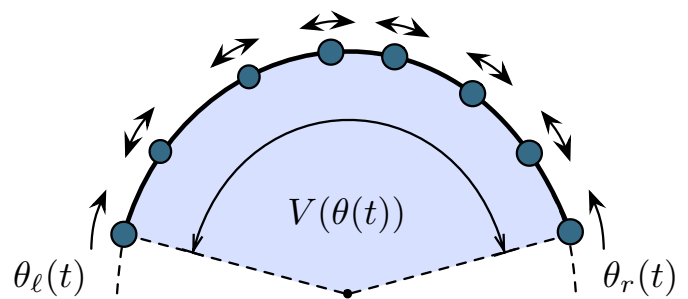

Fig. 11. Illustration of the Lyapunov function candidate $V(\theta(t))$ for angles in an open semicircle $\theta(t) \in \operatorname{Arc}_{n}(\pi)$. The oscillators at the boundaries of the arc containing all oscillators $\overline{\operatorname{Arc}}_{n}\left(V(\theta(t))\right.$ are denoted by $\theta_{\ell}(t)$ and $\theta_{r}(t)$.

distance is attained by more than one pair of oscillators. In summary, we state the following result, which follows from the analysis of nonlinear consensus protocols, see (Lin et al., 2007, Theorem 3.6 and 3.7) and (Moreau, 2005, Theorems 1 and 2) for continuous and discrete-time results.

Theorem 5.2 (Contraction in Open Semicircle $\operatorname{Arc}_{n}(\pi)$ ) Consider the coupled oscillator model (29) with a connected graph $G(\mathcal{V}, \mathcal{E}, A)$ and $\omega=\mathbb{O}_{n}$. Each set $\overline{\operatorname{Arc}}_{n}(\gamma)$, for $\gamma \in[0, \pi[$, is positively invariant, and each trajectory originating in $\overline{\operatorname{Arc}}_{n}(\gamma)$ achieves exponential phase synchronization, that is,

$$
\left\|\theta(t)-\theta_{\mathrm{avg}} \mathbb{1}_{n}\right\|_{2} \leq\left\|\theta(0)-\theta_{\mathrm{avg}} \mathbb{1}_{n}\right\|_{2} e^{\lambda_{\mathrm{ps}} t},
$$

where $\lambda_{\mathrm{ps}}=-\lambda_{2}(L) \operatorname{sinc}(\gamma)$ and $\theta_{\mathrm{avg}}=\sum_{i=1}^{n} \theta_{i}(0) / n$ is the average initial phase. ${ }^{7}$

Theorem 5.2 also holds for directed and time-variant graphs, it applies to more general interaction functions, and it can be extended to time-delayed systems. Applications to oscillator networks and extensions can be found in (Lin et al., 2007; Moreau, 2005; Münz et al., 2009; Ha et al., 2010a; Sarlette, 2009; Ha et al., 2010a; Dörfler and Bullo, 2011, 2012b; Schmidt et al., 2012). We will revisit this literature in Section 6. An elegant generalization of the above analysis to oscillator networks on $n$ spheres (rather than on $\mathbb{S}^{1}$ ) can be found in (Zhu, 2013), and diffusively-coupled Lienard-type oscillators can be analyzed using similar ideas in the phase plane (Tuna, 2012).

Remark 2 (A control-theoretical perspective on synchronization) As established in Theorems 5.1 and 5.2, the phase-synchronized set $\bar{\Delta}_{G}(0)=\overline{\operatorname{Arc}}_{n}(0)$ is locally exponentially stable provided that all natural frequencies are identical. While phase synchronization is not possible for dissimilar natural frequencies, a certain

\footnotetext{
7 This "average" of angles (points on $\mathbb{S}^{1}$ ) is well-defined in an open semi-circle. If the parametrization of $\theta$ has no discontinuity inside the arc containing all angles, then the average can be obtained by the usual formula.
} 

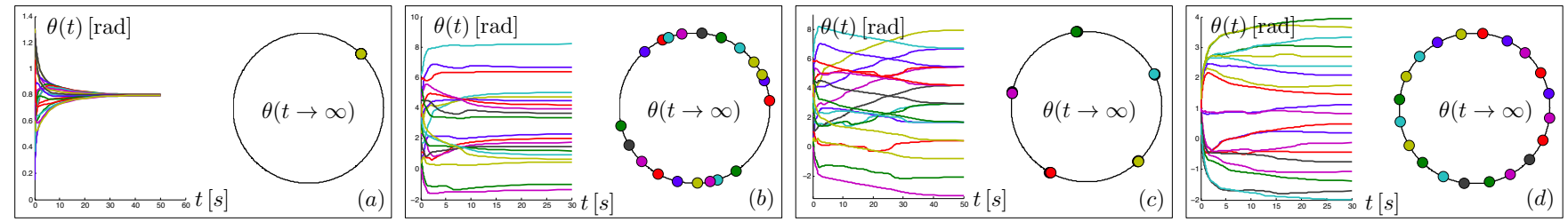

Fig. 12. Synchronization in a network of identical oscillators with $n=20, \omega=\mathbb{O}_{n}$, and a ring graph $G(\mathcal{V}, \mathcal{E}, A)$ with unit weights. Subfigure (a) shows phase synchronization achieved by the oscillator network (29). Subfigure (b) shows phase balancing achieved by the oscillator network (34). Subfigure (c) and (d) show pattern formation achieved by the oscillator network (35) (with gains $K_{\ell}=1$ for $\ell<m$ and $K_{m}=-1$ ) for the symmetric balanced (5,20)-pattern and (20,20) splay state pattern.

degree of phase cohesiveness can still be achieved. Indeed, the coupled oscillator model (1) can be regarded as an exponentially stable system subject to the disturbance $\omega \in \mathbb{1}_{n}^{\perp}$ and synchronization can be studied using classic control-theoretical concepts such as input-to-state stability, practical stability, ultimate boundedness (Khalil, 2002) or their incremental versions (Angeli, 2002). In control-theoretical terminology, phase cohesiveness can then be described as "practical phase synchronization." Compared to prototypical nonlinear control examples, various additional challenges arise in the analysis of the coupled oscillator model (1) due to the bounded and nonmonotone sinusoidal coupling, the compact state space, and the coexistence of multiple equilibria.

\subsection{Phase Balancing and Pattern Formation}

As compared with phase synchronization, only few results are known about phase balancing. This asymmetry may be caused by the fact that phase synchrony is studied in more applications than phase balancing. Moreover, the phase-synchronized set $\overline{\operatorname{Arc}}_{n}(0)$ admits a very simple geometric characterization, whereas the phasebalanced set $\mathrm{Bal}_{n}$ has a complicated structure consisting of multiple disjoint subsets. The number of these subsets grows combinatorially with the number of nodes $n$.

Consider the coupled oscillator model (29). By inverting the direction of time, we obtain

$$
\dot{\theta}_{i}=\sum_{j=1}^{n} a_{i j} \sin \left(\theta_{i}-\theta_{j}\right), \quad i \in\{1, \ldots, n\}
$$

In what follows, we say that an undirected graph $G(\mathcal{V}, \mathcal{E}, A)$ is circulant if the adjacency matrix $A=A^{T}$ is a circulant matrix. Circulant graphs are highly symmetric graphs; examples include complete graphs, complete bipartite graphs, and ring graphs. ${ }^{8}$ For circulant and uniformly weighted graphs, the coupled oscillator model (34) achieves phase balancing. The following theorem summarizes several results originally presented in (Sepulchre et al., 2007, Theorem 1) and (Sepulchre et al., 2008, Theorem 2).

\footnotetext{
8 A gallery and examples of circulant graphs can be found at http://mathworld.wolfram.com/CirculantGraph.html.
}

Theorem 5.3 (Phase balancing) Consider the coupled oscillator model (34) with a connected, undirected, uniformly weighted, and circulant graph $G(\mathcal{V}, \mathcal{E}, A)$. The following statements hold:

1) Local phase balancing: The phase-balanced set $\mathrm{Bal}_{n}$ is locally asymptotically stable; and

2) Almost global stability: If the graph $G(\mathcal{V}, \mathcal{E}, A)$ is complete, then the region of attraction of the stable phase-balanced set $\mathrm{Bal}_{n}$ is almost all of $\mathbb{T}^{n}$.

The proof of Theorem 5.3 is similar to that of Theorem 5.1: convergence is established by a potential function argument and the local (in)stability of equilibria is established by a Jacobian argument. An illustrative simulation is shown in Fig. 12(b). The analysis leading to (Sepulchre et al., 2008, Theorem 2) suggests also almost global stability of the phase-balanced set for arbitrary circulant graphs, but a complete proof is not available. For non-circulant graphs, the conclusions of Theorem 5.3 are not true. As a remedy to achieve almost globally stable phase balancing, higher order models with dynamic coupling can be considered, see (Scardovi et al., 2007; Sepulchre et al., 2008) for further details.

Alternatively, phase balancing can also be achieved by coupling functions with higher-order harmonics. For example, a modification of model (34) is

$$
\dot{\theta}_{i}=\sum_{j=1}^{n} \sum_{\ell=1}^{m} \frac{K_{\ell} \cdot a_{i j}}{\ell} \sin \left(\ell\left(\theta_{i}-\theta_{j}\right)\right)
$$

where $K_{\ell} \in \mathbb{R}$ are appropriate gains, and $m \in \mathbb{N}$ divides $n$. The dynamics (35) are again a gradient system whose critical points include symmetric balanced $(m, n)$ patterns; recall Fig. 9 for a schematic illustration. The following result is given in (Sepulchre et al., 2007, Theorem 7) and (Sepulchre et al., 2008, Theorem 7).

Theorem 5.4 (Pattern formation) Consider the coupled oscillator model (35) with a connected, undirected, uniformly weighted, and circulant graph $G(\mathcal{V}, \mathcal{E}, A)$. Let $m \in \mathbb{N}$ be a divisor of $n$, let $K_{\ell}>0$ for $\ell \in\{1, \ldots, m-1\}$, and let $K_{m}<0$ be sufficiently small. Then each symmetric balanced $(m, n)$-pattern is a locally exponentially stable equilibrium manifold. 
Two representative simulations of the pattern-forming model (35) are shown in Fig. 12(c) and Fig. 12(d). Theorem 5.4 can also be extended to non-circulant (and directed) graphs through dynamic coupling, see (Sepulchre et al., 2008, Theorem 8). Notice that Theorem 5.4 establishes only the local stability of $(m, n)$ patterns; the dynamics (35) may feature also other stable equilibria. For the complete graph, Sepulchre et al. (2007) conjecture almost global convergence to the set of symmetric balanced $(m, n)$-patterns.

\section{Synchronization in Complete Networks}

In this section, we study heterogeneous oscillators coupled in a complete graph with uniform weights $a_{i j}=K / n$. In this case, the coupled oscillator model (1) reduces to the celebrated Kuramoto model given in (3). The Kuramoto model will reach synchronization provided that the coupling gain $K$ is larger than a critical value, which depends on the dissimilarity among the natural frequencies $\omega$. Starting from Winfree's and Kuramoto's pioneering work (Winfree, 1967; Kuramoto, 1975, 1984a), this trade-off has been characterized by parametric inequalities. In what follows, we review various estimates of the critical coupling strength to characterize the on-set of synchronization as well as the ultimate stage of synchronization. We consider both infinite-dimensional as well as finite-dimensional Kuramoto models.

\subsection{Infinite-Dimensional Kuramoto Models}

In his ingenious analysis of the continuum-limit model (18)-(19) Kuramoto considered continuous, even, and unimodal distributions $g(\omega)$ of the natural frequencies (achieving their maximum at $g(0)$ ), and found that partially-synchronized solutions (if existent) must satisfy the self-consistency equation (Kuramoto, 1984a)

$$
r=K r \int_{-\pi / 2}^{\pi / 2} \cos ^{2}(\theta) g(K r \sin (\theta)) d \theta .
$$

One trivial solution to the self-consistency equation (36) is $r=0$ corresponding to the uniform incoherent state shown in Fig. 10(a). The second solution for $r>0$ corresponds to the partially-synchronized state illustrated in Fig. 10(b). When canceling the variable $r$ from both sides of (36) and taking the limit $r \searrow 0$, the self-consistency equation (36) delivers the bifurcation parameter

$$
K_{\text {partial }}=\frac{2}{\pi g(0)}
$$

Kuramoto conjectured that the uniform incoherent state would become unstable for $K>K_{\text {partial }}$ and concluded famously that "surprisingly enough, this seemingly obvious fact seems difficult to prove." The resolution of this long-standing conjecture and Kuramoto's ingenious yet incomplete analysis inspired generations of scientists, see (Strogatz, 2000) for an historical account. We present the following general result by (Chiba, 2014, Theorem 3.5).

Theorem 6.1 (Instability of the incoherent state) Consider the infinite-dimensional Kuramoto model (18)(19) with coupling gain $K$ and frequency distribution $g: \mathbb{R} \rightarrow \mathbb{R}_{\geq 0}$. Assume that the number of roots $\left\{y_{1}, y_{2}, \ldots\right\}$ of the equation

$$
\lim _{x \searrow 0} \int_{-\infty}^{\infty} \frac{\omega-y}{x^{2}+(w-y)^{2}} g(\omega) d \omega=0,
$$

is countable, and $g(\omega)$ is continuous at $\left\{y_{1}, y_{2}, \ldots\right\}$. If

$$
K>K_{\text {partial }}=\frac{2}{\pi \sup _{j} g\left(y_{j}\right)}
$$

then the incoherent state $\rho(\theta, t, \omega)=1 /(2 \pi)$ is unstable.

Notice that Theorem 6.1 is fairly general and includes bimodal distributions. It can be shown that for a continuous, even, and unimodal distribution $g(\omega)$, the unique root of (38) is given by $y_{1}=0$, see (Chiba, 2014, Corollary 3.6). This observation leads to the following corollary, which can be found in (Crawford, 1994; Balmforth and Sassi, 2000; Acebrón et al., 2005; Mirollo and Strogatz, 2007; Ott and Antonsen, 2008; Martens et al., 2009; Chiba, 2014), and references therein.

Corollary 6.2 (Instability beyond Kuramoto's critical transition point) Consider the infinitedimensional Kuramoto model (18)-(19) with coupling gain $K$ and frequency distribution $g: \mathbb{R} \rightarrow \mathbb{R}_{\geq 0}$. Suppose that $g(\omega)$ is continuous at the origin, even, and unimodal. If $K$ is greater than $K_{\text {partial }}$ as given in (37), then the incoherent state $\rho(\theta, t, \omega)=1 /(2 \pi)$ is unstable.

A linear stability analysis of the associated partiallysynchronized state illustrated in Fig. 10(b) is discussed by Mirollo and Strogatz (2007) and reveals linear neutral stability. To the best of the authors' knowledge, a nonlinear stability analysis of the partially-synchronized state is still outstanding.

If the distribution $g(\omega)$ is restricted to have bounded support, then the fully phase-locked state (illustrated in Fig. 10(c)) can be achieved when the coupling $K$ is larger than the second critical threshold $K_{\text {lock }} \geq K_{\text {partial }}$. In this case, two distributions of interest are the uniform distribution and the bipolar distribution given by

$$
\begin{aligned}
g_{\text {unif }}: & {\left[-\omega_{0},+\omega_{0}\right] \rightarrow \mathbb{R}, \quad g_{\text {unif }}(\omega)=\frac{1}{2 \omega_{0}}, } \\
g_{\text {bip }}: & {\left[\omega_{\min }, \omega_{\max }\right] \rightarrow \mathbb{R}, } \\
& g_{\text {bip }}(\omega)=p \cdot \delta\left(\omega-\omega_{\max }\right)+(1-p) \cdot \delta\left(\omega-\omega_{\text {min }}\right),
\end{aligned}
$$



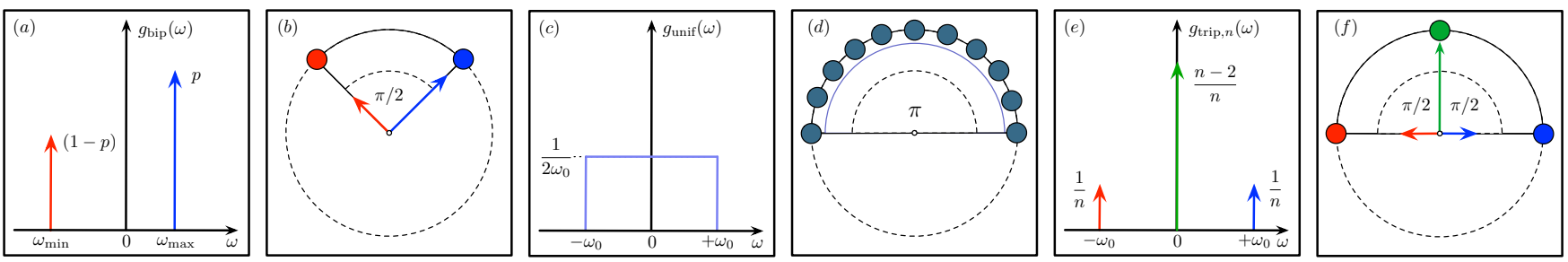

Fig. 13. Extremal distributions $g(\omega)$ of the natural frequencies and their stationary phase distributions in the critical case $K \searrow K_{\text {lock}}$ : Panels (a) and (b) show the (generally non-symmetric) bipolar distribution $g_{\text {bip }}(\omega)=p \cdot \delta\left(\omega-\omega_{\max }\right)+(1-p) \cdot \delta\left(\omega-\omega_{\min }\right)$ and its associated bipolar phase distribution. Panels (c) and (d) show the uniform distribution $g_{\text {unif }}(\omega)=1 /\left(2 \omega_{0}\right)$ and its associated uniform phase distribution. Finally, panels (e) and (f) show the tripolar distribution $g_{\text {trip }, n}(\omega)=\frac{1}{n} \delta\left(\omega-\omega_{0}\right)+\frac{n-2}{n} \delta(\omega)+\frac{1}{n} \delta\left(\omega+\omega_{0}\right)$ and its associated tripolar phase distribution for $n \rightarrow \infty$.

where $\omega_{0}>0, \omega_{\max }>\omega_{\min }$, and $p \in[0,1]$. These two distributions are particularly interesting since they yield the smallest and the largest threshold $K_{\text {lock }}$.

\section{Theorem 6.3 (Full phase locking thresholds)}

Consider the infinite-dimensional Kuramoto model (18)(19) with coupling gain $K$ and frequency distribution $g: \mathbb{R} \rightarrow \mathbb{R}_{>0}$ with bounded support. The following statements hold for the full phase-locking threshold $K_{\text {lock}}$ :

(i) Lower bound: For any continuous, even, and unimodal $g:\left[-\omega_{0},+\omega_{0}\right] \rightarrow \mathbb{R}$, where $\omega_{0}>0$, we have $K_{\text {lock }} \geq 4 \omega_{0} / \pi$. Moreover, for the uniform distribution $g_{\text {unif }}(\omega)$, we have $K_{\text {lock }}=4 \omega_{0} / \pi$.

(ii) Upper bound: For any $g:\left[\omega_{\min }, \omega_{\max }\right] \rightarrow \mathbb{R}_{\geq 0}$, where $\omega_{\max }>\omega_{\min }$, we have $K_{\text {lock }} \leq \omega_{\max }-\omega_{\min }$. Moreover, for the bipolar distribution $g_{\mathrm{bip}}(\omega)$, we have $K_{\text {lock }}=\omega_{\max }-\omega_{\min }$.

A proof of the lower bound (i) can be found in (Ermentrout, 1985, Corollary 2(b)) and (Mirollo and Strogatz, $2007)$. Notice that the two thresholds $K_{\text {partial }}$ (reported in (37)) and $K_{\text {lock }}$ coincide for the uniform distribution:

$$
K_{\text {lock }}=\frac{2}{\pi g_{\text {unif }}(0)}=K_{\text {partial }}
$$

This remarkable identity was also observed by Hemmen and Wreszinski (1993); Mirollo and Strogatz (2007); Roberts (2008); Verwoerd and Mason (2011). The upper bound (ii) on bipolar distributions has been proved by Hemmen and Wreszinski (1993) and earlier by Ermentrout (1985) for the symmetric case $\left(p=1 / 2\right.$ and $\omega_{\max }=$ $\left.-\omega_{\min }=\omega_{0}\right)$. Bipolar and more general bimodal frequency distributions $g(\omega)$ have attracted tremendous research interest by dynamical system researchers thanks to their rich bifurcation diagram, see (Acebrón et al., 2005; Martens et al., 2009). The uniform and bipolar distributions are shown in Fig. 13 together with the associated stationary phase distributions in the critical case $K \searrow K_{\text {lock }}$ (explicitly calculated by Hemmen and Wreszinski (1993)). For later reference, Fig. 13 also shows the tripolar distribution $g_{\text {trip }, n}(\omega)=\frac{1}{n} \delta\left(\omega-\omega_{0}\right)+$ $\frac{n-2}{n} \delta\left(\omega_{0}\right)+\frac{1}{n} \delta\left(\omega+\omega_{0}\right)$ and its associated phase distri- bution (calculated by Chopra and Spong (2009, Proof of Theorem 2.1)) for the case $n \rightarrow \infty$.

\subsection{Finite-Dimensional Kuramoto Models}

In the finite-dimensional case, various necessary, sufficient, implicit, and explicit estimates of the critical coupling strength $K_{\text {critical }}$ have been proposed (Hemmen and Wreszinski, 1993; Aeyels and Rogge, 2004; Jadbabaie et al., 2004; Acebrón et al., 2005; Mirollo and Strogatz, 2005; De Smet and Aeyels, 2007; Chopra and Spong, 2009; Verwoerd and Mason, 2008, 2009; Chung and Slotine, 2010; Ha et al., 2010a; Ha and Slemrod, 2011; Choi et al., 2011a; Franci et al., 2011; Dörfler and Bullo, 2011, 2012b; Schmidt et al., 2012). We refer to (Dörfler and Bullo, 2011) for a comprehensive historical overview and present only the best known results here.

Necessary, explicit, and tight conditions: The necessary condition (28) evaluated for $\gamma \nearrow \pi / 2$ gives the following lower bound for the critical coupling:

$$
K \geq K_{\text {critical }} \triangleq \frac{n \cdot\left(\omega_{\max }-\omega_{\min }\right)}{2(n-1)} .
$$

Of course, this often-reported lower bound (39) is generally conservative. The following tighter lower bound has been constructed by Chopra and Spong (2009). Here, we report a refined formulation of their necessary condition.

Lemma 6.4 (Explicit, necessary, and tight critical coupling) Consider the Kuramoto model (3) with $n \geq 2$ oscillators, natural frequencies $\omega \in \mathbb{1}_{n}^{\perp}$, and coupling strength $K$. Define $\gamma \in[\pi / 2, \pi]$ by

$$
\gamma=2 \operatorname{arcos}\left(\frac{-(n-2)+\sqrt{(n-2)^{2}+32}}{8}\right) .
$$

The Kuramoto model has a frequency-synchronized solution only if the coupling strength $K$ is larger than a critical value, that is,

$$
K \geq K_{\text {critical }} \triangleq \frac{n \cdot\left(\omega_{\max }-\omega_{\min }\right)}{2(\sin (\gamma)+(n-2) \sin (\gamma / 2))} .
$$


Moreover, condition (41) is tight: for $\omega=\omega_{\text {trip }} \triangleq \omega_{0}$. $\left(+1,-1, \mathbb{O}_{n-2}\right)$ with $\omega_{0} \in \mathbb{R}$ and for any of its permutations, there exists a synchronous solution if and only if $K \geq K_{\text {critical }}$.

Notice that the bound (41) equals the bound (39) for $n=2$ and for $n \rightarrow \infty$, and it is a strict improvement otherwise. The bound (41) is reported in (Chopra and Spong, 2009, Eqs. (8) and (11)) and is computed using optimization techniques. Though not explicitly stated by Chopra and Spong (2009), it can be verified from their proof that the lower bound (40)-(41) is tight for $\omega=\omega_{\text {trip. }}$. In the critical case $K=K_{\text {critical }}$, the associated arc-invariant equilibrium manifold is given by $\left[\theta^{*}\right]=\left[\left(+\gamma / 2,-\gamma / 2, \mathbb{D}_{n-2}\right)\right]$. In the limit $n \rightarrow \infty$ this choice of natural frequencies $\omega$ corresponds to the tripolar distribution in Fig. 13(e), and the associated phases $\left[\theta^{*}\right]$ are shown in Fig. $13(\mathrm{f})$.

Exact and implicit conditions: The articles (Aeyels and Rogge, 2004; Mirollo and Strogatz, 2005; Verwoerd and Mason, 2008) derive a set of implicit consistency equations for the exact critical coupling strength $K_{\text {critical }}$ for which frequency-synchronized solutions exist. The consistency equation can be easily motivated. Each equilibrium solution to the Kuramoto model (16)(17) is characterized by $\left[\theta^{*}\right] \in \mathbb{T}^{n}$ such that the righthand side of (17) equals zero. We denote the corresponding value of the order parameter $(16)$ by $r_{\infty} \in[0,1]$ and, without loss of generality, we assume that its phase $\psi$ is zero. Hence, we arrive at the equations

$$
\begin{aligned}
\omega_{i} & =K r_{\infty} \sin \left(\theta_{i}^{*}\right), \\
r_{\infty} & =\frac{1}{n} \sum_{j=1}^{n} \cos \left(\theta_{i}^{*}\right) .
\end{aligned}
$$

The equations (42) are solvable only if $K r_{\infty} \geq\|\omega\|_{\infty}$, and thus necessarily $r_{\infty}>0$ unless $\omega=\mathbb{O}_{n}$. By eliminating $\theta^{*}$ from (42), we arrive at the consistency equation

$$
r_{\infty}=\frac{1}{n} \sum_{j=1}^{n} \pm \sqrt{1-\left(\omega_{i} / K r_{\infty}\right)^{2}}
$$

where the \pm signs are due to the equality: $\cos (\arcsin (x))=$ $\pm \sqrt{1-x^{2}}$ for $\left.x \in\right]-1,1[$. In fact, the consistency equation (43) is a set of $2^{n}$ equations corresponding to different possible equilibria $\theta^{*}$ in (42) and thus different choices of the \pm signs, although not all choices yield feasible solutions $r_{\infty} \geq 0$. We refer to (Aeyels and Rogge, 2004) for a discussion of the consistency equation (43) and its infinite-dimensional counterpart (36). The implicit consistency equation (43) marks the starting point for the analyses in (Aeyels and Rogge, 2004; Mirollo and Strogatz, 2005; Verwoerd and Mason, 2008). By collecting various results in these three references, we arrive at the following statement, which has not been presented in this complete and self-contained form so far.
Theorem 6.5 (Implicit formulae for the exact critical coupling) Consider the Kuramoto model (3) with $n \geq 2$ oscillators, natural frequencies $\omega \in \mathbb{1}_{n}^{\perp} \backslash\left\{\mathbb{O}_{n}\right\}$, and coupling strength $K$. Compute $u^{*} \in\left[\|\omega\|_{\infty}, 2\|\omega\|_{\infty}\right]$ as unique solution to the equation

$$
2 \sum_{i=1}^{n} \sqrt{1-\left(\omega_{i} / u\right)^{2}}=\sum_{i=1}^{n} 1 / \sqrt{1-\left(\omega_{i} / u\right)^{2}} .
$$

The following statements are equivalent:

(i) Critical coupling: the coupling strength $K$ is larger than a critical value, that is,

$$
K>K_{\text {critical }} \triangleq n u^{*} / \sum_{i=1}^{n} \sqrt{1-\left(\omega_{i} / u^{*}\right)^{2}}
$$

(ii) Stable frequency synchronization: there exists a locally exponentially stable equilibrium manifold $\left[\theta^{*}\right] \subset \mathbb{T}^{n}$.

The implicit formulae (44)-(45) have been established by Verwoerd and Mason (2008, Theorem 3), who showed that $K_{\text {critical }}$ is the smallest nonnegative value of the coupling strength for which the Kuramoto model (3) admits a frequency-synchronized solution. We remark that Verwoerd and Mason also extended the implicit formulae (44)-(45) to complete bipartite graphs (Verwoerd and Mason, 2009, Theorem 3) and infinite-dimensional networks (Verwoerd and Mason, 2011, Theorem 4). Moreover, they provided bisection algorithms to compute $K_{\text {critical }}$ with predefined precision in a finite number of iterations. Aeyels and Rogge (2004) and Mirollo and Strogatz (2005) found similar implicit formulae and carried out a local stability analysis (Aeyels and Rogge, 2004, Theorems 1 and 3) and (Mirollo and Strogatz, 2005, Sections 3 and 4) showing a saddle-node bifurcation for $K=K_{\text {critical }}$ : for $K<K_{\text {critical }}$ no frequency-synchronized solution (i.e., equilibrium manifolds) exists and for $K>K_{\text {critical }}$ a locally exponentially stable (corresponding to all + signs in (43)) and multiple unstable phase-locked solutions co-exist. As shown by Roberts (2008), the Kuramoto model (3) can be embedded into a higher-dimensional, linear, and complex-valued system, ${ }^{9}$ and the above stability results can also be elegantly established via linear systems theory; see also the recent work by Conteville and Panteley (2012); El Ati and Panteley (2013a,b).

Sufficient, explicit, and tight conditions: For the purpose of analyzing and selecting a sufficiently strong coupling in applications, Theorem 6.5 has three drawbacks. The stability results are local and the region of attraction of a synchronized solution is unknown. Second, the exact formulae (44)-(45) are implicit and thus not suited for performance estimates. For example, it is

\footnotetext{
9 An embedding of the Kuramoto model (3) in a Hamiltonian system can be found in (Witthaut and Timme, 2013).
} 
unclear which value of asymptotic arc invariance can be achieved if $K>c \cdot K_{\text {critical }}$ for some $c>1$. Third and finally, the natural frequencies $\omega_{i}$ are often time-varying or uncertain in most applications. In this case, the exact value of $K_{\text {critical }}$ needs to be estimated in continuous time, or a conservatively strong coupling $K \gg K_{\text {critical }}$ has to be chosen. The following theorem provides an explicit upper bound on the critical coupling together with performance estimates, convergence rates, and a guaranteed semi-global region of attraction. This bound is tight and thus necessary and sufficient when considering arbitrary distributions with compact support of the natural frequencies. The result has been originally presented in (Dörfler and Bullo, 2011, Theorem 4.1).

Theorem 6.6 (Explicit, sufficient, and tight critical coupling and practical phase sync) Consider the Kuramoto model (3) with $n \geq 2$ oscillators, natural frequencies $\omega \in \mathbb{1}_{n}^{\perp}$ and coupling strength $K$. The following statements are equivalent:

(i) Critical coupling: the coupling strength $K$ is larger than a critical value, that is,

$$
K>K_{\text {critical }} \triangleq \omega_{\max }-\omega_{\min }
$$

(ii) Admissible initial arc invariance: there exists $\left.\left.\gamma_{\max } \in\right] \pi / 2, \pi\right]$ such that the Kuramoto model (3) achieves exponential frequency synchronization for all possible distributions of the natural frequencies $\omega_{i}$ supported on the compact interval $\left[\omega_{\min }, \omega_{\max }\right]$ and for all initial phases $\theta(0) \in \operatorname{Arc}_{n}\left(\gamma_{\max }\right)$; and

(iii) Arc invariance of sync manifold: there exists $\gamma_{\min } \in[0, \pi / 2[$ such that the Kuramoto model (3) has a locally exponentially stable synchronization manifold in $\overline{\operatorname{Arc}}_{n}\left(\gamma_{\text {min }}\right)$ for all possible distributions of the natural frequencies $\omega_{i}$ supported on the compact interval $\left[\omega_{\min }, \omega_{\max }\right]$.

If the equivalent conditions (i), (ii), and (iii) hold, then the ratio $K_{\text {critical }} / K$ and the arc lengths $\gamma_{\min } \in[0, \pi / 2[$ and $\left.\left.\gamma_{\max } \in\right] \pi / 2, \pi\right]$ are related uniquely via $\sin \left(\gamma_{\min }\right)=$ $\sin \left(\gamma_{\max }\right)=K_{\text {critical }} / K$. Moreover, the Kuramoto model (3) achieves practical phase synchronization, that is, the set $\overline{\operatorname{Arc}}_{n}(\gamma)$ is positively invariant for every $\gamma \in\left[\gamma_{\min }, \gamma_{\max }\right]$, and each trajectory originating in $\operatorname{Arc}_{n}\left(\gamma_{\text {max }}\right)$ approaches asymptotically $\overline{\operatorname{Arc}}_{n}\left(\gamma_{\text {min }}\right)$.

The proof of Theorem 6.6 relies on the Jacobian and contraction properties developed in Subsections 4.1 and 5.2. If all angles at time $t \geq 0$ belong to a closed arc of length $\gamma \in\left[0, \pi\left[\right.\right.$, that is, $\bar{\theta}(t) \in \overline{\operatorname{Arc}}_{n}(\gamma)$, then the arc length $t \mapsto V(\theta(t))$ given in (32) is non-increasing if

$$
K \sin (\gamma) \geq \omega_{\max }-\omega_{\min } .
$$

The above inequality holds true for $\gamma \in\left[\gamma_{\min }, \gamma_{\max }\right]$ if and only if condition (46) holds true. Additionally, $t \mapsto$
$V(\theta(t))$ is strictly decreasing for $\gamma \in] \gamma_{\min }, \gamma_{\max }[$, the angles $\theta(t)$ reach the set $\overline{\operatorname{Arc}}_{n}\left(\gamma_{\text {min }}\right) \in \Delta_{G}(\pi / 2)$, and frequency synchronization and stability follow from the results developed in Subsection 4.1. Hence, condition (46) implies properties (ii) and (iii) of Theorem 6.6. The converse implications follow since condition (46) is also necessary for synchronization with bipolar natural frequen$\operatorname{cies} \omega=\omega_{\text {bip }} \triangleq \omega_{0} \cdot\left(-p \cdot \mathbb{1}_{n-p},+(n-p) \cdot \mathbb{1}_{p}\right)$ with $\omega_{0} \in \mathbb{R}$, $p \in\{1, \ldots, n-1\}$, and for any of its permutations. In recent work, (Choi et al., 2013) show that the bipolar distribution $\omega_{\text {bip }}$ is the unique worst-case distribution, where synchronization fails for $K=K_{\text {critical }}$.

Besides establishing a tight condition for $K_{\text {critical }}$, Theorem 6.6 establishes some properties of the transient evolution of the Kuramoto dynamics (3) and shows that the asymptotic synchronization behavior of the Kuramoto model (3) is best described by the terminology practical phase synchronization, see also (Franci et al., 2011; Dörfler and Bullo, 2011). Notice also that Theorem 6.6 reduces to Theorem 5.2 for identical natural frequencies. We remark that similar analysis results are reported in (De Smet and Aeyels, 2007; Ha et al., 2010a; Choi et al., 2011a; Dörfler and Bullo, 2012b; Schmidt et al., 2012), and the bound $\gamma_{\min }$ on the ultimate phase distances can be improved for particular pairs of oscillators, see (Choi et al., 2011a, Theorem 5.2). Finally, we remark that the proof strategy via the contraction Lyapunov function (32) can be adapted to more general cases, for example, the conclusions of Theorem 6.6 can be extended to time-varying natural frequencies, see (Dörfler and Bullo, 2011) and the illustration in Fig. 14.

Comparison and statistical analysis: Theorem 6.6 states the tight and explicit upper bound (46) on the critical coupling strength $K_{\text {critical }}$. Likewise, Lemma 6.4 states the tight and explicit lower bound (41) on $K_{\text {critical }}$. The exact critical coupling lies somewhere in-between and can be obtained from the implicit formulae (44)(45). By collecting these results, we can state the following corollary, which improves upon the explicit bounds proposed by Verwoerd and Mason (2008, Corollary 7).

Corollary 6.7 (Tight explicit bounds) Consider the Kuramoto model (3) with $n \geq 2$ oscillators, natural frequencies $\omega \in \mathbb{1}_{n}^{\perp} \backslash\left\{\mathbb{O}_{n}\right\}$, and coupling strength $K$. Compute the exact critical coupling $K_{\text {critical }}$ according to (44)(45). The explicit necessary condition (41) and sufficient condition (46) provide tight upper and lower bounds on the exact critical coupling $K_{\text {critical }}$, that is,

$\frac{n \cdot\left(\omega_{\max }-\omega_{\min }\right)}{2(\sin (\gamma)+(n-2) \sin (\gamma / 2))} \leq K_{\text {critical }} \leq \omega_{\max }-\omega_{\min }$

where $\gamma \in[\pi / 2, \pi]$ is defined in (40). Moreover, the lower bound is tight for $\omega=\omega_{\text {trip }} \triangleq \omega_{0} \cdot\left(+1,-1, \mathbb{D}_{n-2}\right)$, and the upper bound is tight for $\omega=\omega_{\text {bip }} \triangleq \omega_{0} \cdot\left(-p \cdot \mathbb{1}_{n-p},+(n-\right.$ $\left.p) \cdot \mathbb{1}_{p}\right)$, where $\omega_{0} \in \mathbb{R}, p \in\{1, \ldots, n-1\}$, and both $\omega_{\text {trip }}$ 

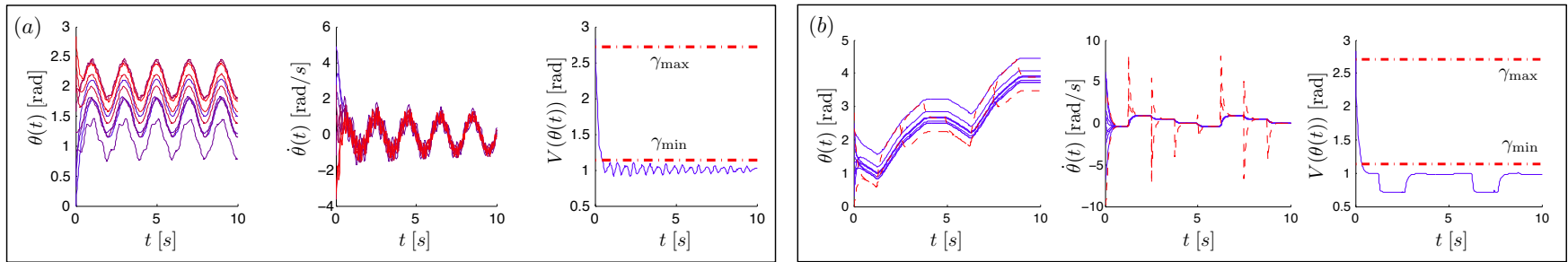

Fig. 14. Simulation of a network of $n=10$ Kuramoto oscillators satisfying $K=1.1 \cdot\left(\omega_{\max }-\omega_{\min }\right)$. In panel (a), the natural frequencies $\omega_{i}: \mathbb{R}_{\geq 0} \rightarrow\left[\omega_{\min }, \omega_{\max }\right]=[0,1]$ are smooth, bounded, and distinct sinusoidal functions. Each natural frequency $\omega_{i}(t)$ asymptotically converges to $\tilde{\omega}_{i}+\sin (\pi t)$ with constant and randomly chosen $\tilde{\omega}_{i} \in[0,1]$. In panel (b), the natural frequencies $\omega_{i}(t)$ of oscillators 1 and 10 (displayed in red dashed lines) switch between constant values in $\left[\omega_{\min }, \omega_{\max }\right]=[0,1]$. The simulations illustrate the phase cohesiveness of the angles $\theta(t)$ in $\overline{\operatorname{Arc}}_{n}\left(\gamma_{\min }\right)$, the boundedness and convergence of the frequency variations (between consecutive switching instances) $\dot{\theta}(t)-\omega_{\text {avg }}(t) \mathbb{1}_{n}$, as well as the monotonicity of the Lyapunov function $V(\theta(t))$ in $\overline{\operatorname{Arc}}_{n}(\gamma)$ for $\gamma \in\left[\gamma_{\min }, \gamma_{\max }\right]$.

and $\omega_{\mathrm{bip}}$ are defined modulo index permutations.

Corollary 6.7 is the finite-dimensional counterpart to Theorem 6.3 and identifies bipolar and tripolar frequency distributions $\omega_{\text {bip }}$ and $\omega_{\text {trip }}$ as the extreme choices for the resulting critical coupling $K_{\text {critical }}{ }^{10}$ These two distributions of natural frequencies are illustrated in Fig. 13(a) and 13(e). We want to remark that for natural frequencies sampled from a particular distribution, $g(\omega)$, the critical quantity in Corollary 6.7, the support $\omega_{\max }-\omega_{\min }$, can be estimated by extreme value statistics, see (Bronski et al., 2012) for further details.

By Theorem 6.3, for infinite-dimensional models the uniform distribution $g_{\text {unif }}(\omega)=1 /\left(2 \omega_{0}\right)$ yields the smallest synchronization threshold $K_{\text {lock }}=4 \omega_{0} / \pi$ over all continuous, symmetric, and unimodal distributions $g(\omega)$ with bounded support $\omega \in\left[-\omega_{0},+\omega_{0}\right]$. Hence, the uniform distribution is an interesting choice to compare the three conditions (41), (44)-(45), and (46) in a statistical analysis. Fig. 15 reports our numeric findings for $\omega_{0}=1$.

All three displayed conditions are identical for $n=2$ oscillators. As $n$ increases, the sufficient condition (46) converges to the width $\omega_{\max }-\omega_{\min }=2 \omega_{0}$ of the support of $g_{\text {unif }}(\omega)$, and the necessary condition (41) converges to half of that width. The exact value of $K_{\text {critical }}$ given by (44)-(45) converges to $4\left(\omega_{\max }-\omega_{\min }\right) /(2 \pi)=$ $4 \omega_{0} / \pi$ in agreement with condition (37) predicted for the continuum-limit model (18)-(19).

\section{$7 \quad$ Synchronization in Sparse Networks}

This section considers the coupled oscillator model (1) in its general form featuring de-synchronizing dissimilar natural frequencies $\omega \in \mathbb{1}_{n}^{\perp}$ and the synchronizing coupling through a graph $G(\mathcal{V}, \mathcal{E}, A)$ with a nontrivial

\footnotetext{
${ }^{10}$ Notice that the extreme choices for the lower bounds in Theorem 6.3 and Corollary 6.7 do not coincide, since $g(\omega)$ is required to be continuous for the lower bound in Theorem 6.3.
}

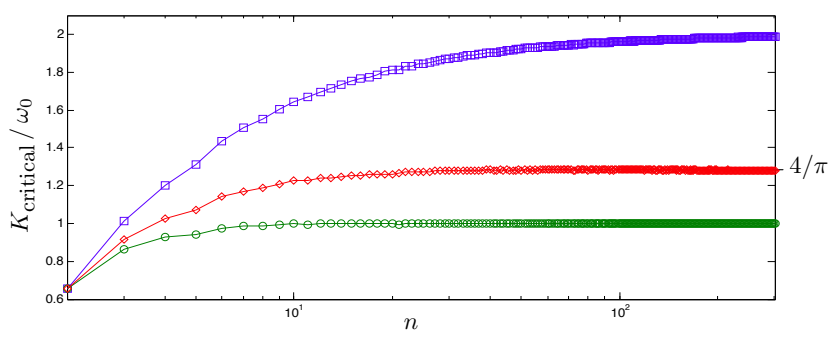

Fig. 15. Statistical analysis of the necessary, tight, and explicit bound $(41)(\diamond)$, the exact and implicit formulae (44)-(45) (O), and the sufficient, tight, and explicit bound (46) ( $\square$ ) for $n \in[2,300]$ oscillators, where the coupling gains for each $n$ are averaged over 1000 samples of randomly uniformly generated frequencies in the interval $[-1,1]$.

topology. The network science, nonlinear dynamics, and physics communities coined the term complex for such non-trivial topologies to distinguish them from longrange (complete) and short-range (lattice-type) interaction topologies. The interest in such complex oscillator networks has been sparked by the seminal article (Jadbabaie et al., 2004) and the widespread scientific attention given to complex network studies (Strogatz, 2001; Boccaletti et al., 2006; Osipov et al., 2007; Arenas et al., 2008; Suykens and Osipov, 2008; Dorogovtsev et al., 2008), and consensus and its applications (Olfati-Saber et al., 2007; Ren et al., 2007; Bullo et al., 2009; Garin and Schenato, 2010; Mesbahi and Egerstedt, 2010).

\subsection{Survey of Synchronization Metrics and Conditions}

Loosely speaking, the oscillator network (1) achieves synchronization when the coupling dominates the dissimilarity in natural frequencies. Various conditions have been proposed to quantify this trade-off for sparse graphs, both in theoretical studies as well as in power network applications. The coupling is typically quantified by the algebraic connectivity $\lambda_{2}(L)(\mathrm{Wu}$ and Kumagai, 1980; Pecora and Carroll, 1998; Nishikawa et al., 2003; Jadbabaie et al., 2004; Restrepo et al., 2005; Boccaletti et al., 2006; Arenas et al., 2008; Dörfler and 
Bullo, 2012b; Motter et al., 2013), the weighted nodal degree $\operatorname{deg}_{i}=\sum_{j=1}^{n} a_{i j}$ (Wu and Kumagai, 1982; Korniss et al., 2006; Gómez-Gardeñes et al., 2007; Buzna et al., 2009; Dörfler and Bullo, 2012b, 2013a; Skardal et al., 2013), or various metrics related to the notion of effective resistance (Wu and Kumagai, 1982; Korniss et al., 2006; Dörfler and Bullo, 2013a). The frequency dissimilarity is quantified either by absolute norms $\|\omega\|_{p}$ or by incremental norms ${ }^{11}\left\|B^{T} \omega\right\|_{p}$, for $p \in \mathbb{N}$. Here, we specifically consider the three incremental norms:

$$
\begin{aligned}
\|\omega\|_{\mathcal{E}, \infty} & \triangleq\left\|B^{T} \omega\right\|_{\infty}=\max _{\{i, j\} \in \mathcal{E}}\left|\omega_{i}-\omega_{j}\right| \\
\|\omega\|_{\mathcal{E}, 2} & \triangleq\left\|B^{T} \omega\right\|_{2}=\left(\sum_{\{i, j\} \in \mathcal{E}}\left|\omega_{i}-\omega_{j}\right|^{2}\right)^{1 / 2}, \\
\|\omega\|_{\mathcal{E}_{\mathrm{cplt}, 2}} & \triangleq\left\|B_{\mathrm{cplt}}^{T} \omega\right\|_{2}=\frac{1}{2}\left(\sum_{i, j=1}^{n}\left|\omega_{i}-\omega_{j}\right|^{2}\right)^{1 / 2}
\end{aligned}
$$

where the subscript cplt stands for the complete graph. With slight abuse of notation, we also adopt these incremental norms for angular distances. For example, for $\gamma \in$ $\left[0, \pi\left[\right.\right.$, the incremental $\infty$-norm ball $\left\{\theta \in \mathbb{T}^{n} \mid\|\theta\|_{\mathcal{E}_{\infty}} \leq\right.$ $\gamma\}$ is identical to the phase cohesive set $\bar{\Delta}_{G}(\gamma)$.

As every review article on synchronization (Strogatz, 2000, 2001; Acebrón et al., 2005; Boccaletti et al., 2006; Arenas et al., 2008; Dorogovtsev et al., 2008; Dörfler et al., 2013), let us state here that the problem of finding sharp and provably correct synchronization conditions is not yet completely solved. Some of the proposed synchronization conditions for complex phase oscillator networks can be evaluated only numerically since they are state-dependent (Wu and Kumagai, 1980, 1982) or arise from a non-trivial linearization process of full state space oscillator models. The latter procedure is adopted in the widely-studied Master Stability Function formalism, see (Pecora and Carroll, 1998; Boccaletti et al., 2006; Arenas et al., 2008) for the original reference and relevant surveys, see (Restrepo et al., 2004; Sun et al., 2009; Sorrentino and Porfiri, 2011) for its extension to quasi-identical oscillators, and see (Shafi et al., 2013; Russo and Di Bernardo, 2009) for related linearizationbased approaches from the control community.

In general, concise and accurate results are known only for specific topologies such as complete graphs (as discussed in the previous section), linear chains (Strogatz and Mirollo, 1988), highly symmetric ring graphs (Buzna et al., 2009), acyclic graphs (Dekker and Taylor, 2013), and complete bipartite graphs (Verwoerd and Mason, 2009) with uniform weights. For arbitrary coupling topologies, the literature contains only sufficient conditions ( $\mathrm{Wu}$ and Kumagai, 1980, 1982; Jadbabaie et al., 2004; Dörfler and Bullo, 2012b) as well as numerical and statistical investigations for large random net-

\footnotetext{
${ }^{11}$ More precisely, the incremental norms $\left\|B^{T} \omega\right\|_{p}$ are seminorms in $\mathbb{R}^{n}$ and proper norms in the quotient space $\mathbb{1}_{n}^{\perp}$.
}

works indicating certain (e.g., degree-dependent) scaling laws (Nishikawa et al., 2003; Restrepo et al., 2005; Gómez-Gardeñes et al., 2007; Moreno and Pacheco, 2004; Kalloniatis, 2010; Skardal et al., 2013).

Numerical studies indicate that all known and provablycorrect synchronization conditions are conservative estimates on the threshold from incoherence to synchrony. Our recently-proposed condition (Dörfler et al., 2013) is provably correct for various extremal network topologies and weights, and is numerically accurate for a broad range of random networks; a complete analytic treatment is missing at this time. In the following, we review a set of known and provably correct synchronization conditions and analysis concepts.

\subsection{Sufficient Synchronization Conditions}

For arbitrary network topologies and weights the equilibrium and potential energy landscape of the oscillator network (1) has been studied by different communities, see (Tavora and Smith, 1972a; Korsak, 1972; Araposthatis et al., 1981; Baillieul and Byrnes, 1982; Mehta and Kastner, 2011). We particularly recommend the article (Araposthatis et al., 1981), where various surprising and counter-intuitive examples are reported. To the best of the authors' knowledge, the conditions (27)-(28) in Lemma 4.2 are the best known explicit necessary conditions for the existence of equilibria for arbitrary topologies and weights. In what follows, we focus on sufficient conditions guaranteeing frequency synchronization, and we restrict ourselves to phase cohesive synchronous solutions within the set $\Delta_{G}(\pi / 2)$. There are two reasons for this choice. First, as discussed in Subsection 4.1, the equilibria in $\Delta_{G}(\pi / 2)$ are exponentially stable, and the forward invariance of the set $\Delta_{G}(\pi / 2)$ leads to stable synchronization by incremental stability or frequency dynamics arguments. Second, from a pragmatic point of view, there are few analysis results and conditions for equilibria outside $\Delta_{G}(\pi / 2)$, with the treatment of (directed) ring graphs in (Rogge and Aeyels, 2004; Ha and Kang, 2012) being a notable exception.

The approaches to phase synchronization (in Section 5) and to frequency synchronization in complete graphs (in Section 6) are generally not applicable to dissimilar natural frequencies and sparse coupling graphs, or are so only under very conservative conditions. For example, in the presence of dissimilar natural frequencies $\omega \in \mathbb{1}_{n}^{\perp}$, a Lyapunov analysis of the forced system (26) via the trigonometric potential function $U(\theta)$ is very involved since the level sets of $U(\theta)$ are hard to characterize. Likewise, the contraction Lyapunov analysis based on definition (32) inherently requires arc-invariance of all angles, and does not easily extend to arbitrary topologies. One quadratic Lyapunov function advocated by Jadbabaie et al. (2004); Chopra and Spong (2009) for classic Ku- 
ramoto oscillators (3) is $W: \operatorname{Arc}_{n}(\pi) \rightarrow \mathbb{R}$ defined by

$$
W(\theta)=\frac{1}{4} \sum_{i, j=1}^{n}\left|\theta_{i}-\theta_{j}\right|^{2}=\frac{1}{2}\|\theta\|_{\mathcal{E}_{\mathrm{cplt}}, 2}^{2}
$$

This Lyapunov function is useful to analyze the more general oscillator network (1), and yields the following result found in (Dörfler and Bullo, 2012a, Theorem 4.6) and (Dörfler and Bullo, 2012b, Theorem 4.4).

Theorem 7.1 (Practical phase synchronization in sparse graphs I) Consider the coupled oscillator model (1) with a connected graph $G(\mathcal{V}, \mathcal{E}, A)$ and frequencies $\omega \in \mathbb{1}_{n}^{\perp}$. There exists a locally exponentially stable equilibrium manifold $[\theta] \in \Delta_{G}(\pi / 2)$ if the algebraic connectivity is larger than a critical value, that is,

$$
\lambda_{2}(L)>\lambda_{\text {critical }} \triangleq\|\omega\|_{\mathcal{E}_{\text {cplt }}, 2} .
$$

Moreover, if condition (49) holds, then the coupled oscillator model (1) achieves practical phase synchronization in the following sense. Given $\gamma_{\max } \in$ ]$\pi / 2, \pi]$ and $\gamma_{\min } \in[0, \pi / 2[$ as unique solutions to $(\pi / 2) \cdot \operatorname{sinc}\left(\gamma_{\max }\right)=\sin \left(\gamma_{\min }\right)=\lambda_{\text {critical }} / \lambda_{2}(L)$, the set $\left\{\theta \in \operatorname{Arc}_{n}(\pi) \mid\|\theta\|_{\mathcal{E}_{\mathrm{cplt}}, 2}^{2} \leq \gamma\right\} \subseteq \bar{\Delta}_{G}(\gamma)$ is positively invariant for all $\gamma \in\left[\gamma_{\min }, \gamma_{\max }\right]$, and each trajectory starting in $\left\{\theta \in \operatorname{Arc}_{n}(\pi) \mid\|\theta\|_{\mathcal{E}_{\mathrm{cplt}}, 2}^{2}<\gamma_{\text {max }}\right\}$ asymptotically reaches $\left\{\theta \in \operatorname{Arc}_{n}(\pi) \mid\|\theta\|_{\mathcal{E}_{\mathrm{cplt}}, 2}^{2} \leq \gamma_{\text {min }}\right\}$.

The analysis leading to Theorem 7.1 is similar to the proof of Theorem 6.6: the Lyapunov function (48) is used to guarantee the ultimate boundedness of the phases in $\left\{\theta \in \operatorname{Arc}_{n}(\pi) \mid\|\theta\|_{\mathcal{E}_{\text {cplt }}, 2} \leq \gamma_{\text {min }}\right\} \subset \bar{\Delta}_{G}\left(\gamma_{\text {min }}\right)$, and the Jacobian arguments in Subsection 4.1 guarantee frequency synchronization. For classic Kuramoto oscillators (3), condition (49) reduces to $K>\|\omega\|_{\mathcal{E}_{\text {cplt }}, 2}$; this condition is more conservative than the tight bound (46) which reads $K>\|\omega\|_{\mathcal{E}, \infty}=\omega_{\max }-\omega_{\min }$. One reason for this conservatism is that condition (49) guarantees that all phase differences $\left|\theta_{i}-\theta_{j}\right|$ are bounded, not only those along the edges of the graph. However, by Lemma 4.1, we know that bounded phase differences $\left|\theta_{i}-\theta_{j}\right|$ only for $\{i, j\} \in \mathcal{E}$, are sufficient to establish the existence of a locally exponentially stable synchronized solution.

In what follows we adopt a fixed-point approach to the study of the equilibrium equations for the coupled oscillator model (1). In matrix notation, these equilibrium equations read as

$$
\omega=B \mathcal{A} \sin \left(B^{T} \theta\right),
$$

where $\mathcal{A}=\operatorname{diag}\left(\left\{a_{i j}\right\}_{\{i, j\} \in \mathcal{E}}\right)$ is the diagonal matrix of weights. We next follow the ingenious analysis of (50) suggested in (Jadbabaie et al., 2004, Section IIV.B). For the sake of a streamlined presentation, we treat the angles $\theta$ as vectors in $\mathbb{1}_{n}^{\perp}$. Recall the state-dependent weights $c_{i j}(\theta)=a_{i j} \operatorname{sinc}\left(\theta_{i}-\theta_{j}\right)$ from the consensus formulation (31), and define the state-dependent Laplacian

$$
\mathcal{L}(\theta)=B \operatorname{diag}\left(\left\{c_{i j}(\theta)\right\}_{\{i, j\} \in \mathcal{E}}\right) B^{T} .
$$

Hence, equations (50) can be written compactly as $\omega=$ $\mathcal{L}(\theta) \theta$. Since $\mathcal{L}(\theta)^{\dagger} \cdot \mathcal{L}(\theta)=I_{n}-\frac{1}{n} \mathbb{1}_{n \times n}$, we arrive at

$$
\theta=\mathcal{L}(\theta)^{\dagger} \omega
$$

The following result has been obtained in (Dörfler and Bullo, 2012a, Theorem 4.7) by applying to equation (51) a fixed point theorem in the incremental two norm $\|\cdot\|_{\mathcal{E}, 2}$.

Theorem 7.2 (Practical phase synchronization in sparse graphs II) Consider the coupled oscillator model (1) with a connected graph $G(\mathcal{V}, \mathcal{E}, A)$ and frequencies $\omega \in \mathbb{1}_{n}^{\perp}$. There exists a locally exponentially stable equilibrium manifold $\left[\theta^{*}\right] \in \Delta_{G}(\pi / 2)$ if

$$
\lambda_{2}(L)>\lambda_{\text {critical }} \triangleq\|\omega\|_{\mathcal{E}, 2} .
$$

Moreover, if condition (52) holds, then $\left[\theta^{*}\right]$ is phase cohesive in the following sense: $\left[\theta^{*}\right] \subset\left\{\theta \in \mathbb{T}^{n} \mid\|\theta\|_{\mathcal{E}, 2} \leq\right.$ $\left.\gamma_{\min }\right\} \subseteq \bar{\Delta}_{G}\left(\gamma_{\min }\right)$, where $\gamma_{\min } \in[0, \pi / 2[$ satisfies $\sin \left(\gamma_{\min }\right)=\lambda_{\text {critical }} / \lambda_{2}(L)$.

Clearly, condition (52) is sharper than condition (49), but the stability result is only local. The synchronization condition (52) is the sharpest sufficient condition for general graphs known to the authors, but it is still a conservative estimate for most network topologies and weights. Indeed, the necessary condition (28) and sufficient condition (52) are separated by a tremendous gap for $n>2$ oscillators. The reasons for this conservatism are manifold. First, the derivation of the conditions (27), (28), (49), and (52) involves conservative bounding of the trigonometric nonlinearities and network interactions. Second, by Theorem 7.2, the condition (52) guarantees that the 2-norm of all phase distances between neighboring oscillators is bounded as $\left\|\theta^{*}\right\|_{\mathcal{E}, 2} \leq \arcsin \left(\lambda_{\text {critical }} / \lambda_{2}(L)\right)$. On the other hand, to conclude frequency synchronization by Lemma 4.1, phase synchronization by Theorem 5.2 , or frequency synchronization (in the complete graph case) by Theorem 6.6 , only the worst-case phase distance (between neighbors) $\|\theta\|_{\mathcal{E}, \infty}$ needs to be bounded. We conclude that the incremental 2-norm metric leads to overly strong phase cohesiveness requirements and accordingly to conservative conditions. All our previous results hint at the incremental $\infty$-norm as a natural metric.

\subsection{Towards an Exact Synchronization Condition}

An analysis of the fixed-point equations (51) using 2norm bounding of $\left\|\mathcal{L}(\theta)^{\dagger} \omega\right\|_{\mathcal{E}, 2}$ results in the condition $\|\omega\|_{\mathcal{E}, 2} / \lambda_{2}(L)<1$ in Theorem 7.2. As discussed above, an $\infty$-norm analysis of equations (51) and the term 
$\left\|\mathcal{L}(\theta)^{\dagger} \omega\right\|_{\mathcal{E}, \infty}$ should yield a less conservative condition, possibly of the form $\left\|L^{\dagger} \omega\right\|_{\mathcal{E}, \infty}<1$. Indeed, this condition can be derived for particular networks. By formally replacing each term $\sin \left(\theta_{i}-\theta_{j}\right)$ in the fixed-point equations $(50)$ by an auxiliary scalar variable $\psi_{i j}$ we arrive at

$$
\begin{aligned}
& \omega=B \mathcal{A} \psi, \\
& \psi=\sin \left(B^{T} \theta\right),
\end{aligned}
$$

where $\psi \in \mathbb{R}^{|\mathcal{E}|}$ is a vector with elements $\psi_{i j}$. We refer to equations (53) as the auxiliary-fixed point equation. It can be easily verified that every solution of the auxiliary fixed-point equations (53) is of the form

$$
\psi=B^{T} L^{\dagger} \omega+\psi_{\mathrm{hom}}
$$

where the homogeneous solution $\psi_{\text {hom }} \in \mathbb{R}^{|\mathcal{E}|}$ satisfies $\mathcal{A} \psi_{\text {hom }} \in \operatorname{Ker}(B)$. Note that the orthogonal vector spaces $\operatorname{Ker}(B)$ and $\operatorname{Ker}(B)^{\perp}=\operatorname{Im}\left(B^{T}\right)$ are spanned by vectors associated to cycles and cutsets in the graph, see (Biggs, 1994, 1997). For $x, y \in \mathbb{R}^{n}$, we say $x=y \bmod 2 \pi$ if, for each $i \in\{1, \ldots, n\}$, there exists an integer $k_{i}$ such that $x_{i}=y_{i}+2 \pi k_{i}$. We now arrive at the following characterization of the fixed points (Dörfler et al., 2013, Theorem 1).

Lemma 7.3 (Properties of the fixed point equations) Consider the coupled oscillator model (1) with graph $G(\mathcal{V}, \mathcal{E}, A)$ and $\omega \in \mathbb{1}_{n}^{\perp}$, its fixed-point equations (50), and the auxiliary fixed-point equations (53). Let $\gamma \in[0, \pi / 2[$. The following statements are equivalent:

(i) There exists a solution $\theta^{*} \in \bar{\Delta}_{G}(\gamma)$ to the fixed-point equations (50); and

(ii) There exists a solution $\psi \in \mathbb{R}^{|\mathcal{E}|}$ to the auxiliary fixed-point equation (53) of the form (55) satisfying the norm constraint $\|\psi\|_{\infty} \leq \sin (\gamma)$ and the cycle constraint $\arcsin (\psi)=B^{T} y \bmod 2 \pi$, for some $y \in$ $\mathbb{R}^{n}$.

If the equivalent statements ( $i$ ) and (ii) are true, then we have $B^{T} \theta^{*}=\arcsin (\psi) \bmod 2 \pi$. Additionally, $\left[\theta^{*}\right] \subset$ $\bar{\Delta}_{G}(\gamma)$ is a locally exponentially stable synchronization manifold.

By Lemma 7.3, the cycle space $\operatorname{Ker}(B)$ of the graph serves as a degree of freedom to find a minimum $\infty$-norm solution $\psi^{*} \in \mathbb{R}^{|\mathcal{E}|}$ to equations (53), which yields an optimal necessary synchronization condition.

Corollary 7.4 (Optimal necessary synchronization condition) Consider the coupled oscillator model (1) with a connected graph $G(\mathcal{V}, \mathcal{E}, A)$ and $\omega \in \mathbb{1}_{n}^{\perp}$. Compute $\psi^{*} \in \mathbb{R}^{|\mathcal{E}|}$ as solution to the optimization problem

$$
\operatorname{minimize}_{\psi \in \mathbb{R}^{|\mathcal{E}|}}\|\psi\|_{\infty} \quad \text { s.t. } \quad \omega=B \mathcal{A} \psi \text {. }
$$

Let $\gamma \in[0, \pi / 2[$. There exists a locally exponentially stable equilibrium manifold $\left[\theta^{*}\right] \subset \bar{\Delta}_{G}(\gamma)$ only if $\left\|\psi^{*}\right\|_{\infty} \leq$ $\sin (\gamma)$.

If the graph is acyclic, then there are no cycle constraints, and the norm constraint in Lemma 7.3 reduces to $\sin (\gamma) \geq\|\psi\|_{\infty}=\left\|L^{\dagger} \omega\right\|_{\mathcal{E}, \infty}$. We arrive at the following corollary (Dörfler et al., 2013, Theorem 2).

Corollary 7.5 (Practical phase synchronization in acyclic graphs) Consider the coupled oscillator model (1) with a connected and acyclic graph $G(\mathcal{V}, \mathcal{E}, A)$ and $\omega \in \mathbb{1}_{n}^{\perp}$. There exists a locally exponentially stable equilibrium manifold $\left[\theta^{*}\right] \subset \Delta_{G}(\pi / 2)$ if and only if

$$
\left\|L^{\dagger} \omega\right\|_{\mathcal{E}, \infty}<1
$$

Moreover, if condition (57) holds, then $\left[\theta^{*}\right]$ is phase cohesive in $\bar{\Delta}_{G}\left(\gamma_{\min }\right)$, where $\gamma_{\min } \in[0, \pi / 2[$ satisfies $\sin \left(\gamma_{\min }\right)=\left\|L^{\dagger} \omega\right\|_{\mathcal{E}, \infty}$.

Condition (57) is equivalent to the cutset condition (Dekker and Taylor, 2013, Lemma 1). Dörfler et al. (2013) also proved that condition (57) is sufficient and tight for various extremal graph topologies and parameters such as complete and uniformly weighted graphs (in this case (57) is equivalent to (46)), small cycles of length strictly less than five, cutset-inducing natural frequencies $\omega=L \omega_{\text {bip }}$, in the limit $\left\|L^{\dagger} \omega\right\|_{\mathcal{E}, \infty} \searrow 0$, and 1-connected combinations of these graphs. Moreover, by means of a statistical analysis, it can be shown that condition (57) is extremely accurate for a broad set of random network topologies and weights as well as for standard power network test cases (Dörfler et al., 2013; Dörfler and Bullo, 2013b). However, the authors also identified possibly thin sets of topologies and parameters for which condition (57) is not sufficiently tight.

We conclude this section with a comparison of the synchronization conditions (27), (28), (49), (52), and (57). Let $V \in \mathbb{R}^{n \times n}$ be the matrix of orthonormal eigenvectors of $L$ and let $0=\lambda_{1}<\lambda_{2} \leq \cdots \leq \lambda_{n}$ be the corresponding eigenvalues. Then condition (57) reads as

$$
\left\|V \operatorname{diag}\left(0,1 / \lambda_{2}, \ldots, 1 / \lambda_{n}\right) \cdot\left(V^{T} \omega\right)\right\|_{\mathcal{E}, \infty}<1
$$

A sufficient condition for inequality (58) is $\lambda_{2}>\|\omega\|_{\mathcal{E}, \infty}$, which strictly improves upon the algebraic connectivity conditions (49) and (52). Likewise, a necessary condition for (58) is $2 \cdot \max _{i \in \mathcal{V}} \operatorname{deg}_{i} \geq \lambda_{n} \geq\|\omega\|_{\mathcal{E}, \infty}$, resembling the degree-dependent conditions (27) and (28). When compared to (58), this sufficient condition and this necessary condition feature only one of $n-1$ nonzero Laplacian eigenvalues and are overly conservative. We conclude that condition (57) strongly improves upon the conditions (27), (28), (49), and (52), but a complete analytic characterization of its applicability is still open. 


\section{Conclusions and Open Research Directions}

In this paper we introduced the reader to the coupled oscillator model (1), we reviewed several applications, we discussed different synchronization notions, and we presented different analysis approaches and results for phase synchronization, phase balancing, pattern formation, frequency synchronization, and partial synchronization. We covered complete and sparse network topologies, homogeneous and heterogeneous natural frequencies, and finite and infinite oscillator populations.

Despite the vast literature, the countless applications, and the numerous theoretical results on the synchronization properties of model (1), many interesting and important problems are still open. In the following, we summarize limitations of the existing analysis approaches and present a few worthwhile directions for future research.

Generalized interactions: Most of the results presented in this paper can be extended to more general anti-symmetric and $2 \pi$-periodic coupling functions as long as the coupling is diffusive and bidirectional. In some applications, the coupling topology is inherently directed, such as transcriptional, metabolic, or neuronal networks (Mason and Verwoerd, 2007). In this case, there are only a few theoretical investigations including analyses for ring graphs (Rogge and Aeyels, 2004; Ha and Kang, 2012) and acyclic graphs (Ha and Li, 2014), results on the synchronization frequency (El Ati and Panteley, 2013a; Dörfler and Bullo, 2012b), and statistical analysis of large graphs (Restrepo et al., 2006). Also, in many applications the diffusive coupling includes a phase shift (Izhikevich, 1998). For instance, mutual excitatory or inhibitory synaptic organizations in neuroscience (Crook et al., 1997), time delays in sensor networks (Simeone et al., 2008), or transfer conductances in power networks (Chiang et al., 1995) lead to a shifted coupling of the form $\sin \left(\theta_{i}-\theta_{j}-\varphi_{i j}\right)$ with $\varphi_{i j} \in[-\pi / 2, \pi / 2]$. In these cases and also for other "skewed" or "symmetry-breaking" interactions among the oscillators, many of the presented analysis schemes either fail or lead to overly conservative results. Additionally, the inclusion of odd coupling functions possibly with higher-order harmonics can lead to qualitatively different behavior and scaling laws as discussed by Daido (1994); Crawford (1995); Strogatz (2000). Finally, a topic of recent interest is mixed attractive and repulsive coupling (Hong and Strogatz, 2011; El Ati and Panteley, 2013b; Burylko, 2012).

Pulse coupling: Another interesting class of oscillator networks are systems of pulse-coupled oscillators which were introduced by Peskin (1975) and popularized in the seminal work by Mirollo and Strogatz (1990). Pulsecoupled oscillators feature hybrid dynamics: impulsive coupling at discrete time instants and uncoupled con- tinuous dynamics otherwise. This class of oscillator networks displays a very interesting phenomenology which is qualitatively different from diffusive and continuous coupling, see (Mauroy et al., 2012). For instance, the behavior of identical oscillators coupled in a complete graph strongly depends on the curvature of the uncoupled dynamics. As discussed in Subsection 2.4, weakly pulse-coupled oscillator models can be reduced to the canonical model (14) through a phase reduction and averaging analysis. For certain weakly pulse-coupled oscillators the coupling functions $h_{i j}(\cdot)$ turn out to be monotone and discontinuous, and they result in finite-time convergent dynamics (Mauroy and Sepulchre, 2012; Kuramoto, 1991). Most of the results and analysis methods known for continuously-coupled oscillators still need to be extended to pulse-coupled oscillators, especially in the case of dissimilar natural frequencies.

Transient dynamics: For dissimilar oscillators, most results presented in this paper pertain to existence and local stability of synchronous solutions, with the exception of Theorems 6.6 and 7.1. Even for the Kuramoto model (3), many problems pertaining to the transient dynamics still need to be fully resolved. For instance, most known estimates on the region of attraction of a synchronized solution are conservative, such as the semicircle estimates given in Theorems 5.2 and 6.6. We refer to (Chiang et al., 1995; Wiley et al., 2006) for a set of interesting results and conjectures on the region of attraction. Of further interest is whether almost global frequency synchronization can be achieved for heterogeneous oscillator networks coupled in, e.g., acyclic graphs. As shown in Theorem 6.6, for complete graphs, the region of attraction of a synchronous solution always includes $\Delta_{G}(\pi / 2)$ for any $K>K_{\text {critical }}$. It is unclear if an analogous result holds for sparse graphs or if the region of attraction severely depends on the topology. When the Kuramoto dynamics (3) are subject to additive noise, they can be analyzed through Fokker-Planck equations similar to the continuum-limit model (18)-(19) or in the limit of small stochastic perturbations, see (Bag et al., 2007; DeVille, 2011). In this case, there are various interesting transitions between wells of the potential landscape and only few analytic investigations. Also the sub-synchronous regime for $K<K_{\text {critical }}$ is vastly unexplored, and partial synchronization or clustering (similar to the infinite-dimensional model) (Aeyels and Rogge, 2004; De Smet and Aeyels, 2007), chimera states (Laing, 2009; Martens et al., 2013), or chaotic motion (Maistrenko et al., 2005; Tönjes, 2007; Popovych et al., 2005; Suykens and Osipov, 2008) can occur. Finally, the incremental stability results referenced in Subsection 4.1 appear to be a promising direction that still needs to be fully explored.

Higher-order phase oscillator networks: For the mechanical analog in Fig. 1 and the previously listed applications (Bergen and Hill, 1981; Ermentrout, 1991; Chiang et al., 1995; Sauer and Pai, 1998; Wiesenfeld 
et al., 1998; Hoppensteadt and Izhikevich, 2000; Bennett et al., 2002; Pantaleone, 2002; Strogatz et al., 2005; Righetti and Ijspeert, 2006; Shim et al., 2007; Ha et al., 2010b, 2011; Kapitaniak et al., 2012; Zhang et al., 2012) the coupled oscillator dynamics are not exactly given by the first-order phase model (1). In many cases, the dynamics are of second order as in (9). The analysis of second-order oscillator networks has also received a lot of attention, see (Acebrón et al., 2005; Dörfler and Bullo, 2011; Choi et al., 2011b) for a literature overview. Among others, the contraction Lyapunov function (32) can be extended to second-order oscillators (Choi et al., 2011b), the continuum-limit analysis can be extended (Acebrón et al., 2005), and the local stability properties are preserved when going from first to second order (Dörfler and Bullo, 2011). Of course, the transient dynamics of second-order oscillator networks have their own characteristics, especially for large inertia and small damping (Paganini and Lesieutre, 1999). Thus, many of the presented results still need to be extended to secondorder oscillator networks.

State space and aperiodic oscillator networks: In other instances of oscillator networks, there is no readilyavailable phase variable to describe the periodic limitcycle dynamics of the coupled system, and the phase oscillator model is valid only after a phase reduction and averaging analysis. Since features of the original model may be poorly preserved in the phase model (4), a direct analysis of the state space model is preferred. In the case of linear or passive systems, state or output synchronization are well understood, see for example, (Arcak, 2007; Wieland, 2010; Bürger et al., 2013; Lunze, 2012). The analysis of synchronization problems in general periodic and heterogeneous state space oscillator networks remains a challenging and important problem. Additionally, synchronization phenomena can also occur among chaotic and aperiodic oscillators (Pecora and Carroll, 1990), whose analysis is thus far mainly restricted to numerical linearization via the Master Stability Function approach (Pecora and Carroll, 1998; Boccaletti et al., 2006; Arenas et al., 2008; Motter et al., 2013). It is yet unclear which analysis methods carry over from phase oscillator networks to state space or chaotic oscillator networks.

Sparse and heterogeneous networks: Despite the vast scientific interest the quest for sharp, concise, and closed-form synchronization conditions for arbitrary connected graphs has been so far in vain. As suggested by our discussion in Section 7, the proper metric for the analysis of synchronization problem appears to be the incremental $\infty$-norm. In the authors' opinion, an analysis with the incremental $\infty$-norm will most likely deliver the sharpest possible conditions. We believe that the norm and cycle constraints developed in (Dörfler et al., 2013) are a fruitful approach towards a more complete understanding of sparse topologies. Likewise, for the transient analysis, the $\ell_{\infty}$-type contraction Lyapunov function (32) is a powerful analysis concepts for complete graphs and still needs to be extended to arbitrary connected graphs. Regarding the potential and equilibrium landscape, a few interesting and still unresolved conjectures can be found in Tavora and Smith (1972a); Araposthatis et al. (1981); Baillieul and Byrnes (1982); Mehta and Kastner (2011); Korsak (1972) and pertain to the number of (stable) equilibria and topological properties of the equilibrium set. Finally, the complex networks, nonlinear dynamics, and statistical physics communities found various interesting scaling laws in their statistical and numerical analyses of random graph models, such as conditions depending on the spectral ratio $\lambda_{2} / \lambda_{n}$ of the Laplacian eigenvalues, interesting results for correlations between the degree $\operatorname{deg}_{i}$ and the natural frequency $\omega_{i}$, and degree-dependent synchronization conditions (Nishikawa et al., 2003; Moreno and Pacheco, 2004; Restrepo et al., 2005; Boccaletti et al., 2006; Gómez-Gardeñes et al., 2007; Arenas et al., 2008; Kalloniatis, 2010; Skardal et al., 2013). It is unclear which of these results and findings are amenable to an analytic and quantitative investigation.

We sincerely hope that this survey article stimulates further exciting research on synchronization in coupled oscillators, both on the theoretical side as well as in the countless applications.

\section{Acknowledgments}

The authors are grateful to the anonymous reviewers for their detailed comments and for refining the scope of this survey. The authors also acknowledge helpful discussions and insightful comments by many colleagues, including Asad Abidi, Bruce Francis, Alexandre Mauroy, Rodolphe Sepulchre, John Simpson-Porco, Francesco Sorrentino, and Steven Strogatz.

\section{References}

Abidi, A. A., Chua, L. O., 1979. On the dynamics of Josephson-junction circuits. IEE Journal on Electronic Circuits and Systems 3 (4), 186-200.

Acebrón, J. A., Bonilla, L. L., Vicente, C. J. P., Ritort, F., Spigler, R., 2005. The Kuramoto model: A simple paradigm for synchronization phenomena. Reviews of Modern Physics 77 (1), 137-185.

Adler, R., 1946. A study of locking phenomena in oscillators. Proceedings of the IRE 34 (6), 351-357.

Aeyels, D., Rogge, J. A., 2004. Existence of partial entrainment and stability of phase locking behavior of coupled oscillators. Progress on Theoretical Physics 112 (6), 921-942.

Ainsworth, N., Grijalva, S., 2013. A structure-preserving model and sufficient condition for frequency synchronization of lossless droop inverter-based AC networks. IEEE Transactions on Power Systems 28 (4), 43104319 . 
Angeli, D., 2002. A Lyapunov approach to incremental stability properties. IEEE Transactions on Automatic Control 47 (3), 410-421.

Aoi, S., Tsuchiya, K., 2005. Locomotion control of a biped robot using nonlinear oscillators. Autonomous Robots 19 (3), 219-232.

Appleton, E. V., 1922. Automatic synchronization of triode oscillators. Mathematical Proceedings of the Cambridge Philosophical Society 21 (pt 111), 231.

Araposthatis, A., Sastry, S., Varaiya, P., 1981. Analysis of power-flow equation. International Journal of Electrical Power \& Energy Systems 3 (3), 115-126.

Arcak, M., 2007. Passivity as a design tool for group coordination. IEEE Transactions on Automatic Control 52 (8), 1380-1390.

Arcak, M., Dec. 2012. Synchronization and pattern formation in diffusively coupled systems. In: IEEE Conf. on Decision and Control. Maui, HI, USA, pp. 71847192.

Arenas, A., Díaz-Guilera, A., Kurths, J., Moreno, Y., Zhou, C., 2008. Synchronization in complex networks. Physics Reports 469 (3), 93-153.

Bag, B. C., Petrosyan, K. G., Hu, C. K., 2007. Influence of noise on the synchronization of the stochastic $\mathrm{Ku}$ ramoto model. Physical Review E 76 (5), 056210.

Baillieul, J., Byrnes, C. I., 1982. Geometric critical point analysis of lossless power system models. IEEE Transactions on Circuits and Systems 29 (11), 724-737.

Baldoni, R., Corsaro, A., Querzoni, L., Scipioni, S., Piergiovanni, S. T., 2010. Coupling-based internal clock synchronization for large-scale dynamic distributed systems. IEEE Transactions on Parallel and Distributed Systems 21 (5), 607-619.

Balmforth, N. J., Sassi, R., 2000. A shocking display of synchrony. Physica D: Nonlinear Phenomena 143 (1), 21-55.

Barbarossa, S., Scutari, G., 2007. Decentralized maximum-likelihood estimation for sensor networks composed of nonlinearly coupled dynamical systems. IEEE Transactions on Signal Processing 55 (7), 34563470 .

Bennett, M., Schatz, M. F., Rockwood, H., Wiesenfeld, K., 2002. Huygens's clocks. Proceedings: Mathematical, Physical and Engineering Sciences 458 (2019), 563-579.

Bergen, A. R., Hill, D. J., 1981. A structure preserving model for power system stability analysis. IEEE Transactions on Power Apparatus and Systems 100 (1), 25-35.

Biggs, N., 1994. Algebraic Graph Theory, 2nd Edition. Cambridge University Press.

Biggs, N., 1997. Algebraic potential theory on graphs. Bulletin of the London Mathematical Society 29 (6), 641-683.

Blekhman, I., 1988. Synchronization in Science and Technology. American Society of Mechanical Engineers.

Boccaletti, S., Latora, V., Moreno, Y., Chavez, M., Hwang, D. U., 2006. Complex networks: Structure and dynamics. Physics Reports 424 (4-5), 175-308.

Bronski, J. C., DeVille, L., Jip Park, M., 2012. Fully synchronous solutions and the synchronization phase transition for the finite- $N$ Kuramoto model. Chaos: An Interdisciplinary Journal of Nonlinear Science 22 (3), 033133.

Brown, E., Holmes, P., Moehlis, J., 2003. Globally coupled oscillator networks. In: Kaplan, E., Marsden, J. E., Sreenivasan, K. R. (Eds.), Perspectives and Problems in Nonlinear Science: A Celebratory Volume in Honor of Larry Sirovich. Springer, pp. 183-215.

Brown, E., Moehlis, J., Holmes, P., 2004. On the phase reduction and response dynamics of neural oscillator populations. Neural Computation 16 (4), 673-715.

Buck, J., 1988. Synchronous rhythmic flashing of fireflies. II. Quarterly Review of Biology 63 (3), 265-289.

Bullo, F., Cortés, J., Martínez, S., 2009. Distributed Control of Robotic Networks. Princeton University Press.

Bürger, M., Zelazo, D., Allgöwer, F., 2013. Hierarchical clustering of dynamical networks using a saddle-point analysis. IEEE Transactions on Automatic Control 58 (1), 113-124.

Burylko, O., 2012. Competition and bifurcations in phase oscillator networks with positive and negative couplings. In: Proceedings of the Nonlinear Dynamics of Electronic Systems. Jul., pp. 1-4.

Buzna, L., Lozano, S., Diaz-Guilera, A., 2009. Synchronization in symmetric bipolar population networks. Physical Review E 80 (6), 66120.

Canale, E., Monzón, P., 2008. Almost global synchronization of symmetric Kuramoto coupled oscillators. In: Systems Structure and Control. InTech Education and Publishing, Ch. 8, pp. 167-190.

Canale, E. A., Monzón, P., Robledo, F., Dec. 2010a. On the complexity of the classification of synchronizing graphs. In: Grid and Distributed Computing, Control and Automation. Jeju Island, Korea, pp. 186-195.

Canale, E. A., Monzón, P. A., Robledo, F., Jun. 2010b. The wheels: an infinite family of bi-connected planar synchronizing graphs. In: IEEE Conf. Industrial Electronics and Applications. Taichung, Taiwan, pp. 2204-2209.

Chandrashekhar, K. S., Hill, D. J., 1986. Cutset stability criterion for power systems using a structurepreserving model. International Journal of Electrical Power \& Energy Systems 8 (3), 146-157.

Chiang, H.-D., Chu, C. C., Cauley, G., 1995. Direct stability analysis of electric power systems using energy functions: Theory, applications, and perspective. Proceedings of the IEEE 83 (11), 1497-1529.

Chiba, H., 2014. A proof of the Kuramoto conjecture for a bifurcation structure of the infinite-dimensional $\mathrm{Ku}$ ramoto model. Ergodic Theory and Dynamical SystemsTo appear.

Choi, Y. P., Ha, S. Y., Jung, S., Kim, Y., 2011a. Asymptotic formation and orbital stability of phase-locked states for the Kuramoto model. Physica D: Nonlinear Phenomena 241 (7), 735-754. 
Choi, Y.-P., Ha, S.-Y., Kang, M., Kang, M., 2013. Exponential synchronization of finite-dimensional $\mathrm{Ku}-$ ramoto model at critical coupling strength. Communications in Mathematical Sciences 11 (2), 385-401.

Choi, Y.-P., Ha, S.-Y., Yun, S.-B., 2011b. Complete synchronization of Kuramoto oscillators with finite inertia. Physica D 240 (1), 32-44.

Chopra, N., Spong, M. W., 2009. On exponential synchronization of Kuramoto oscillators. IEEE Transactions on Automatic Control 54 (2), 353-357.

Chung, S. J., Slotine, J. J., Dec. 2010. On synchronization of coupled Hopf-Kuramoto oscillators with phase delays. In: IEEE Conf. on Decision and Control. Atlanta, GA, USA, pp. 3181-3187.

Cohen, M. A., Grossberg, S., 1983. Absolute stability of global pattern formation and parallel memory storage by competitive neural networks. IEEE Transactions on Systems, Man and Cybernetics 13 (5), 815-826.

Conteville, L., Panteley, E., Mar. 2012. Linear reformulation of the Kuramoto model: Asymptotic mapping and stability properties. In: Int. Conf. on Communications, Computing and Control Applications. Hammamet, Tunisia.

Crawford, J. D., 1994. Amplitude expansions for instabilities in populations of globally-coupled oscillators. Journal of Statistical Physics 74 (5), 1047-1084.

Crawford, J. D., 1995. Scaling and singularities in the entrainment of globally coupled oscillators. Physical Review Letters 74 (21), 4341.

Crook, S. M., Ermentrout, G. B., Vanier, M. C., Bower, J. M., 1997. The role of axonal delay in the synchronization of networks of coupled cortical oscillators. Journal of Computational Neuroscience 4 (2), 161172.

Cross, M. C., Hohenberg, P. C., 1993. Pattern formation outside of equilibrium. Reviews of Modern Physics $65(3), 851$.

Daido, H., 1992. Quasientrainment and slow relaxation in a population of oscillators with random and frustrated interactions. Physical Review Letters 68 (7), 1073-1076.

Daido, H., 1994. Generic scaling at the onset of macroscopic mutual entrainment in limit-cycle oscillators with uniform all-to-all coupling. Physical Review Letters 73 (5), 760-763.

De Smet, F., Aeyels, D., 2007. Partial entrainment in the finite Kuramoto-Sakaguchi model. Physica D: Nonlinear Phenomena 234 (2), 81-89.

Dekker, A. H., Taylor, R., 2013. Synchronization properties of trees in the Kuramoto model. SIAM Journal on Applied Dynamical Systems 12 (2), 596-617.

DeVille, L., 2011. Transitions amongst synchronous solutions for the stochastic Kuramoto model. Nonlinearity 25 (5), 1-20.

Do, A. L., Boccaletti, S., Gross, T., 2012. Graphical notation reveals topological stability criteria for collective dynamics in complex networks. Physical Review Letters 108 (19), 194102.

Dörfler, F., Bullo, F., 2011. On the critical coupling for
Kuramoto oscillators. SIAM Journal on Applied Dynamical Systems 10 (3), 1070-1099.

Dörfler, F., Bullo, F., Dec. 2012a. Exploring synchronization in complex oscillator networks. In: IEEE Conf. on Decision and Control. Maui, HI, USA, pp. 7157-7170.

Dörfler, F., Bullo, F., 2012b. Synchronization and transient stability in power networks and non-uniform $\mathrm{Ku}$ ramoto oscillators. SIAM Journal on Control and Optimization 50 (3), 1616-1642.

Dörfler, F., Bullo, F., 2013a. Kron reduction of graphs with applications to electrical networks. IEEE Transactions on Circuits and Systems I: Regular Papers 60 (1), 150-163.

Dörfler, F., Bullo, F., Jul. 2013b. Novel insights into lossless AC and DC power flow. In: IEEE Power \& Energy Society General Meeting. Vancouver, BC, Canada.

Dörfler, F., Chertkov, M., Bullo, F., 2013. Synchronization in complex oscillator networks and smart grids. Proceedings of the National Academy of Sciences 110 (6), 2005-2010.

Dorogovtsev, S. N., Goltsev, A. V., Mendes, J. F. F., 2008. Critical phenomena in complex networks. Reviews of Modern Physics 80 (4), 1275.

El Ati, A., Panteley, E., Dec. 2013a. Asymptotic phase synchronization of Kuramoto model with weighted non-symmetric interconnections: a case study. In: IEEE Conf. on Decision and Control. Florence, Italy, pp. 1319-1324.

El Ati, A., Panteley, E., 2013b. Synchronization of phase oscillators with attractive and repulsive interconnections. In: Int. Conference on Methods and Models in Automation and Robotics. pp. 22-27.

Ermentrout, G. B., 1985. Synchronization in a pool of mutually coupled oscillators with random frequencies. Journal of Mathematical Biology 22 (1), 1-9.

Ermentrout, G. B., 1991. An adaptive model for synchrony in the firefly pteroptyx malaccae. Journal of Mathematical Biology 29 (6), 571-585.

Ermentrout, G. B., Kopell, N., 1984. Frequency plateaus in a chain of weakly coupled oscillators, I. SIAM Journal on Mathematical Analysis 15 (2), 215-237.

Ermentrout, G. B., Kopell, N., 1991. Multiple pulse interactions and averaging in systems of coupled neural oscillators. Journal of Mathematical Biology 29 (3), 195-217.

Filatrella, G., Nielsen, A. H., Pedersen, N. F., 2008. Analysis of a power grid using a Kuramoto-like model. The European Physical Journal B 61 (4), 485-491.

Fioriti, V., Ruzzante, S., Castorini, E., Marchei, E., Rosato, V., 2009. Stability of a distributed generation network using the Kuramoto models. In: Critical Information Infrastructure Security. Lecture Notes in Computer Science. Springer, pp. 14-23.

Forni, F., Sepulchre, R., 2014. A differential Lyapunov framework for contraction analysis. IEEE Transactions on Automatic ControlTo appear. Available at http: //arxiv.org/abs/1208.2943.

Franci, A., Chaillet, A., Panteley, E., Lamnabhi- 
Lagarrigue, F., 2012. Desynchronization and inhibition of Kuramoto oscillators by scalar mean-field feedback. Mathematics of Control, Signals, and Systems 24 (1-2), 169-217.

Franci, A., Chaillet, A., Pasillas-Lépine, W., 2011. Existence and robustness of phase-locking in coupled $\mathrm{Ku}-$ ramoto oscillators under mean-field feedback. Automatica 47 (6), 1193-1202.

Francis, B. A., 2015. Oscillator synchronization. In: Baillieul, J., Samad, T. (Eds.), Encyclopedia of Systems and Control. Springer, to appear.

Garin, F., Schenato, L., 2010. A survey on distributed estimation and control applications using linear consensus algorithms. In: Bemporad, A., Heemels, M., Johansson, M. (Eds.), Networked Control Systems. LNCIS. Springer, pp. 75-107.

Ghosh, A. K., Chance, B., Pye, E. K., 1971. Metabolic coupling and synchronization of NADH oscillations in yeast cell populations. Archives of Biochemistry and Biophysics 145 (1), 319-331.

Gómez-Gardeñes, J., Moreno, Y., Arenas, A., 2007. Paths to synchronization on complex networks. Physical Review Letters 98 (3), 34101.

Ha, S., Lattanzio, C., Rubino, B., Slemrod, M., 2011. Flocking and synchronization of particle models. Quarterly Applied Mathematics 69 (1), 91-103.

Ha, S.-Y., Ha, T., Kim, J.-H., 2010a. On the complete synchronization of the Kuramoto phase model. Physica D: Nonlinear Phenomena 239 (17), 1692-1700.

Ha, S. Y., Jeong, E., Kang, M. J., 2010b. Emergent behaviour of a generalized Viscek-type flocking model. Nonlinearity 23 (12), 3139-3156.

Ha, S. Y., Kang, M. J., 2012. On the basin of attractors for the unidirectionally coupled Kuramoto model in a ring. SIAM Journal on Applied Mathematics 72 (5), 1549-1574.

Ha, S.-Y., Li, Z., 2014. Complete synchronization of Kuramoto oscillators with hierarchical leadership. Communications in Mathematical Sciences 12 (3), 485508.

Ha, S. Y., Slemrod, M., 2011. A fast-slow dynamical systems theory for the Kuramoto type phase model. Journal of Differential Equations 251 (10), 2685-2695.

Hemmen, J. L. V., Wreszinski, W. F., 1993. Lyapunov function for the Kuramoto model of nonlinearly coupled oscillators. Journal of Statistical Physics 72 (1), $145-166$.

Hill, D. J., Chen, G., May 2006. Power systems as dynamic networks. In: IEEE Int. Symposium on Circuits and Systems. Kos, Greece, pp. 722-725.

Hong, H., Strogatz, S. H., 2011. Kuramoto model of coupled oscillators with positive and negative coupling parameters: An example of conformist and contrarian oscillators. Physical Review Letters 106 (5), 054102.

Hong, Y. W., Scaglione, A., 2005. A scalable synchronization protocol for large scale sensor networks and its applications. IEEE Journal on Selected Areas in Communications 23 (5), 1085-1099.

Hoppensteadt, F. C., Izhikevich, E. M., 1997. Weakly
Connected Neural Networks. Springer.

Hoppensteadt, F. C., Izhikevich, E. M., 2000. Synchronization of laser oscillators, associative memory, and optical neurocomputing. Physical Review E 62 (3), 4010-4013.

Hoppensteadt, F. C., Izhikevich, E. M., 2001. Synchronization of MEMS resonators and mechanical neurocomputing. IEEE Transactions on Circuits and Systems I: Fundamental Theory and Applications 48 (2), $133-138$.

Huepe, C., Cadiz, R. F., Colasso, M., Jun. 2012. Generating music from flocking dynamics. In: American Control Conference. Montréal, Canada, pp. 4339 4344.

Huygens, C., 1893. Oeuvres Complètes De Christiaan Huygens. Societe Hollandaise des Sciences, The Hague, Netherlands, M. Nijhoff, ed.

Ichinomiya, T., 2004. Frequency synchronization in a random oscillator network. Physical Review E 70 (2), 026116.

Ijspeert, A. J., 2008. Central pattern generators for locomotion control in animals and robots: A review. Neural Networks 21 (4), 642-653.

Izhikevich, E. M., 1998. Phase models with explicit time delays. Physical Review E 58 (1), 905.

Izhikevich, E. M., 2007. Dynamical Systems in Neuroscience: The Geometry of Excitability and Bursting. MIT Press.

Izhikevich, E. M., Kuramoto, Y., 2006. Weakly coupled oscillators. Encyclopedia of Mathematical Physics 5, 448.

Jadbabaie, A., Motee, N., Barahona, M., Jun. 2004. On the stability of the Kuramoto model of coupled nonlinear oscillators. In: American Control Conference. Boston, MA, USA, pp. 4296-4301.

Jongen, G., Anemüller, J., Bollé, D., Coolen, A. C. C., Perez-Vicente, C., 2001. Coupled dynamics of fast spins and slow exchange interactions in the XY spin glass. Journal of Physics A: Mathematical and General 34 (19), 3957-3984.

Justh, E. W., Krishnaprasad, P. S., 2004. Equilibria and steering laws for planar formations. Systems \& Control Letters 52 (1), 25-38.

Kalloniatis, A. C., 2010. From incoherence to synchronicity in the network Kuramoto model. Physical Review E 82 (6), 066202.

Kapitaniak, M., Czolczynski, K., Perlikowski, P., Stefanski, A., Kapitaniak, T., 2012. Synchronization of clocks. Physics Reports 517, 1-69.

Khalil, H. K., 2002. Nonlinear Systems, 3rd Edition. Prentice Hall.

Kiss, I. Z., Zhai, Y., Hudson, J. L., 2002. Emerging coherence in a population of chemical oscillators. Science 296 (5573), 1676-1678.

Klein, D. J., 2008. Coordinated control and estimation for multi-agent systems: Theory and practice. Ph.D. thesis, University of Washington.

Klein, D. J., Lee, P., Morgansen, K. A., Javidi, T., 2008. Integration of communication and control using dis- 
crete time Kuramoto models for multivehicle coordination over broadcast networks. IEEE Journal on Selected Areas in Communications 26 (4), 695-705.

Kopell, N., Ermentrout, G. B., 1988. Coupled oscillators and the design of central pattern generators. Mathematical Biosciences 90 (1-2), 87-109.

Korniss, G., Hastings, M. B., Bassler, K. E., Berryman, M. J., Kozma, B., Abbott, D., 2006. Scaling in smallworld resistor networks. Physics Letters A 350 (5-6), 324-330.

Korsak, A. J., 1972. On the question of uniqueness of stable load-flow solutions. IEEE Transactions on Power Apparatus and Systems 91 (3), 1093-1100.

Kozyreff, G., Vladimirov, A. G., Mandel, P., 2000. Global coupling with time delay in an array of semiconductor lasers. Physical Review Letters 85 (18), 3809-3812.

Kuramoto, Y., 1975. Self-entrainment of a population of coupled non-linear oscillators. In: Araki, H. (Ed.), Int. Symposium on Mathematical Problems in Theoretical Physics. Vol. 39 of Lecture Notes in Physics. Springer, pp. 420-422.

Kuramoto, Y., 1984a. Chemical Oscillations, Waves, and Turbulence. Springer.

Kuramoto, Y., 1984b. Cooperative dynamics of oscillator community. Progress of Theoretical Physics Supplement 79, 223-240.

Kuramoto, Y., 1991. Collective synchronization of pulsecoupled oscillators and excitable units. Physica D: Nonlinear Phenomena 50 (1), 15-30.

Laing, C. R., 2009. The dynamics of chimera states in heterogeneous Kuramoto networks. Physica D: Nonlinear Phenomena 238 (16), 1569-1588.

Leonard, N. E., Shen, T., Nabet, B., Scardovi, L., Couzin, I. D., Levin, S. A., 2012. Decision versus compromise for animal groups in motion. Proceedings of the National Academy of Sciences 109 (1), 227-232.

Lin, Z., Francis, B., Maggiore, M., 2007. State agreement for continuous-time coupled nonlinear systems. SIAM Journal on Control and Optimization 46 (1), 288-307.

Lindsey, W. C., Ghazvinian, F., Hagmann, W. C., Dessouky, K., 1985. Network synchronization. Proceedings of the IEEE 73 (10), 1445-1467.

Liu, C., Weaver, D. R., Strogatz, S. H., Reppert, S. M., 1997. Cellular construction of a circadian clock: period determination in the suprachiasmatic nuclei. Cell 91 (6), 855-860.

Lohmiller, W., Slotine, J.-J. E., 1998. On contraction analysis for non-linear systems. Automatica 34 (6), 683-696.

Lunze, J., 2011. Complete synchronization of Kuramoto oscillators. Journal of Physics A: Mathematical and Theoretical 44, 425102.

Lunze, J., 2012. Synchronization of heterogeneous agents. IEEE Transactions on Automatic Control 57 (11), $2885-2890$.

Maistrenko, Y. L., Popovych, O. V., Tass, P. A., 2005. Desynchronization and chaos in the Kuramoto model. In: Chazottes, J.-R., Fernandez, B. (Eds.), Dynamics of Coupled Map Lattices and of Related Spatially Extended Systems. Vol. 671 of Lecture Notes in Physics. Springer, pp. 285-306.

Mallada, E., Tang, A., Dec. 2011. Distributed clock synchronization: Joint frequency and phase consensus. In: IEEE Conf. on Decision and Control and European Control Conference. Orlando, FL, USA, pp. 67426747.

Mallada, E., Tang, A., 2014. Synchronization of weakly coupled oscillators: Coupling, delay and topology. Journal of Physics A: Mathematical and TheoreticalTo appear.

Mangesius, H., Hirche, S., Obradovic, D., Jun. 2012. Quasi-stationarity of electric power grid dynamics based on a spatially embedded Kuramoto model. In: American Control Conference. Montréal, Canada, pp. 2159-2164.

Martens, E. A., Barreto, E., Strogatz, S. H., Ott, E., So, P., Antonsen, T. M., 2009. Exact results for the $\mathrm{Ku}-$ ramoto model with a bimodal frequency distribution. Physical Review E 79 (2), 26204.

Martens, E. A., Thutupalli, S., Fourrière, A., Hallatschek, O., 2013. Chimera states in mechanical oscillator networks. Proceedings of the National Academy of Sciences 110 (26), 10563-10567.

Mason, O., Verwoerd, M., 2007. Graph theory and networks in biology. IET Systems Biology 1 (2), 89-119.

Mauroy, A., Sacré, P., Sepulchre, R. J., Dec. 2012. Kick synchronization versus diffusive synchronization. In: IEEE Conf. on Decision and Control. Maui, HI, USA, pp. 7171-7183.

Mauroy, A., Sepulchre, R., 2012. Contraction of monotone phase-coupled oscillators. Systems \& Control Letters 61 (11), 1097-1102.

Mehta, D., Kastner, M., 2011. Stationary point analysis of the one-dimensional lattice Landau gauge fixing functional, aka random phase XY Hamiltonian. Annals of Physics 326 (6), 1425-1440.

Mesbahi, M., Egerstedt, M., 2010. Graph Theoretic Methods in Multiagent Networks. Princeton University Press.

Michaels, D. C., Matyas, E. P., Jalife, J., 1987. Mechanisms of sinoatrial pacemaker synchronization: a new hypothesis. Circulation Research 61 (5), 704-714.

Mirollo, R., Strogatz, S. H., 2007. The spectrum of the partially locked state for the Kuramoto model. Journal of Nonlinear Science 17 (4), 309-347.

Mirollo, R. E., Strogatz, S. H., 1990. Synchronization of pulse-coupled biological oscillators. SIAM Journal on Applied Mathematics 50 (6), 1645-1662.

Mirollo, R. E., Strogatz, S. H., 2005. The spectrum of the locked state for the Kuramoto model of coupled oscillators. Physica D: Nonlinear Phenomena 205 (14), 249-266.

Mirzaei, A., Heidari, M. E., Bagheri, R., Chehrazi, S., Abidi, A. A., 2007. The quadrature LC oscillator: A complete portrait based on injection locking. IEEE Journal of Solid-State Circuits 42 (9), 1916-1932.

Mizumoto, T., Otsuka, T., Nakadai, K., Takahashi, 
T., Komatani, K., Ogata, T., Okuno, H. G., Oct. 2010. Human-robot ensemble between robot thereminist and human percussionist using coupled oscillator model. In: IEEE/RSJ Int. Conf. on Intelligent Robots \& Systems. Taipei, Taiwan, pp. 1957-1963.

Monzón, P., Paganini, F., Dec. 2005. Global considerations on the Kuramoto model of sinusoidally coupled oscillators. In: IEEE Conf. on Decision and Control. San Diego, CA, USA, pp. 3923-3928.

Moreau, L., 2004. Stability of continuous-time distributed consensus algorithms. In: IEEE Conf. on Decision and Control. Nassau, Bahamas, pp. 3998-4003.

Moreau, L., 2005. Stability of multiagent systems with time-dependent communication links. IEEE Transactions on Automatic Control 50 (2), 169-182.

Moreno, Y., Pacheco, A. F., 2004. Synchronization of Kuramoto oscillators in scale-free networks. EPL (Europhysics Letters) 68, 603.

Motter, A. E., Myers, S. A., Anghel, M., Nishikawa, T., 2013. Spontaneous synchrony in power-grid networks. Nature Physics 9, 191-197.

Münz, U., Papachristodoulou, A., Allgöwer, F., 2009. Consensus reaching in multi-agent packet-switched networks with non-linear coupling. International Journal of Control 82 (5), 953-969.

Nabi, A., Moehlis, J., 2011. Single input optimal control for globally coupled neuron networks. Journal of Neural Engineering 8 (6), 065008.

Néda, Z., Ravasz, E., Vicsek, T., Brechet, Y., Barabási, A.-L., 2000. Physics of the rhythmic applause. Physical Review E 61 (6), 6987-6992.

Nishikawa, T., Motter, A. E., Lai, Y. C., Hoppensteadt, F. C., 2003. Heterogeneity in oscillator networks: Are smaller worlds easier to synchronize? Physical Review Letters 91 (1), 14101.

Olfati-Saber, R., 2006. Swarms on sphere: A programmable swarm with synchronous behaviors like oscillator networks. In: IEEE Conf. on Decision and Control. San Diego, CA, USA, pp. 5060-5066.

Olfati-Saber, R., Fax, J. A., Murray, R. M., 2007. Consensus and cooperation in networked multi-agent systems. Proceedings of the IEEE 95 (1), 215-233.

Osipov, G. V., Kurths, J., Zhou, C., 2007. Synchronization in Oscillatory Networks. Springer.

Ott, E., Antonsen, T. M., 2008. Low dimensional behavior of large systems of globally coupled oscillators. Chaos: An Interdisciplinary Journal of Nonlinear Science 18 (3), 037113.

Ott, E., Antonsen, T. M., 2009. Long time evolution of phase oscillator systems. Chaos: An Interdisciplinary Journal of Nonlinear Science 19 (2), 023117.

Paganini, F., Lesieutre, B. C., 1999. Generic properties, one-parameter deformations, and the BCU method. IEEE Transactions on Circuits and Systems I: Fundamental Theory and Applications 46 (6), 760-763.

Paley, D. A., Leonard, N. E., Sepulchre, R., Grunbaum, D., Parrish, J. K., 2007. Oscillator models and collective motion. IEEE Control Systems Magazine 27 (4), 89-105.
Pantaleone, J., 1998. Stability of incoherence in an isotropic gas of oscillating neutrinos. Physical Review D 58 (7), 073002.

Pantaleone, J., 2002. Synchronization of metronomes. American Journal of Physics 70, 992.

Pecora, L. M., Carroll, T. L., 1990. Synchronization in chaotic systems. Physical Review Letters 64 (8), 821824.

Pecora, L. M., Carroll, T. L., 1998. Master stability functions for synchronized coupled systems. Physical Review Letters 80 (10), 2109-2112.

Peskin, C. S., 1975. Mathematical Aspects of Heart Physiology. Courant Institute of Mathematical Sciences.

Pikovsky, A., Rosenblum, M., 2011. Dynamics of heterogeneous oscillator ensembles in terms of collective variables. Physica D: Nonlinear Phenomena 240 (9), 872-881.

Pikovsky, A., Rosenblum, M., Kurths, J., 2003. Synchronization: A Universal Concept in Nonlinear Sciences. Cambridge University Press.

Pluchino, A., Boccaletti, S., Latora, V., Rapisarda, A., 2006a. Opinion dynamics and synchronization in a network of scientific collaborations. Physica A: Statistical Mechanics and its Applications 372 (2), 316-325.

Pluchino, A., Latora, V., Rapisarda, A., 2006b. Compromise and synchronization in opinion dynamics. The European Physical Journal B - Condensed Matter and Complex Systems 50 (1), 169-176.

Popovych, O. V., Maistrenko, Y. L., Tass, P. A., 2005. Phase chaos in coupled oscillators. Physical Review E 71 (6), 065201.

Rahman, M. M. U., Mudumbai, R., Dasgupta, S., Aug. 2011. Consensus based carrier synchronization in a two node network. In: IFAC World Congress. Milan, Italy, pp. 10038-10043.

Rand, R. H., Holmes, P. J., 1980. Bifurcation of periodic motions in two weakly coupled van der Pol oscillators. International Journal of Non-Linear Mechanics 15 (4), 387-399.

Rayleigh, J. W. S. B., 1896. The Theory of Sound. Vol. 2. Macmillan.

Ren, W., Beard, R. W., Atkins, E. M., 2007. Information consensus in multivehicle cooperative control: Collective group behavior through local interaction. IEEE Control Systems Magazine 27 (2), 71-82.

Restrepo, J. G., Ott, E., Hunt, B. R., 2004. Desynchronization waves and localized instabilities in oscillator arrays. Physical Review Letters 93 (11), 114101.

Restrepo, J. G., Ott, E., Hunt, B. R., 2005. Onset of synchronization in large networks of coupled oscillators. Physical Review E 71 (3), 036151.

Restrepo, J. G., Ott, E., Hunt, B. R., 2006. Synchronization in large directed networks of coupled phase oscillators. Chaos: An Interdisciplinary Journal of Nonlinear Science 16 (1), 015107-015107.

Righetti, L., Ijspeert, A. J., May 2006. Programmable central pattern generators: an application to biped locomotion control. In: IEEE Int. Conf. on Robotics 
and Automation. Orlando, FL, USA, pp. 1585-1590.

Roberts, D. C., 2008. Linear reformulation of the Kuramoto model of self-synchronizing coupled oscillators. Physical Review E 77 (3), 031114.

Rogge, J. A., Aeyels, D., 2004. Stability of phase locking in a ring of unidirectionally coupled oscillators. Journal of Physics A: Mathematical and General 37 (46), 11135-11148.

Rohden, M., Sorge, A., Timme, M., Witthaut, D., 2012. Self-organized synchronization in decentralized power grids. Physical Review Letters 109 (6), 064101.

Russo, G., Di Bernardo, M., 2009. Contraction theory and master stability function: Linking two approaches to study synchronization of complex networks. IEEE Transactions on Circuits and Systems II: Express Briefs 56 (2), 177-181.

Russo, G., Di Bernardo, M., Sontag, E. D., 2010. Stability of networked systems: A multi-scale approach using contraction. In: IEEE Conf. on Decision and Control. Atlanta, GA, USA, pp. 6559-6564.

Sacré, P., Sep. 2013. Systems analysis of oscillator models in the space of phase response curves. Ph.D. thesis, University of Liège.

Sacré, P., Sepulchre, R., Apr. 2014. Sensitivity analysis of oscillator models in the space of phaseresponse curves: Oscillators as open systems. IEEE Control Systems MagazineTo appear. Available at http://arxiv.org/abs/1206.4144.

Sarlette, A., Jan. 2009. Geometry and symmetries in coordination control. Ph.D. thesis, University of Liège, Belgium.

Sarlette, A., Sepulchre, R., 2009. Consensus optimization on manifolds. SIAM Journal on Control and Optimization 48 (1), 56-76.

Sarlette, A., Sepulchre, R., 2011. Synchronization on the circle. In: Dubbeldam, J., Green, K., Lenstra, D. (Eds.), The Complexity of Dynamical Systems. Wiley.

Sastry, S., Varaiya, P., 1980. Hierarchical stability and alert state steering control of interconnected power systems. IEEE Transactions on Circuits and Systems 27 (11), 1102-1112.

Sauer, P. W., Pai, M. A., 1998. Power System Dynamics and Stability. Prentice Hall.

Scardovi, L., Dec. 2010. Clustering and synchronization in phase models with state dependent coupling. In: IEEE Conf. on Decision and Control. Atlanta, GA, USA, pp. 627-632.

Scardovi, L., Sarlette, A., Sepulchre, R., 2007. Synchronization and balancing on the $N$-torus. Systems \& Control Letters 56 (5), 335-341.

Schmidt, G. S., Papachristodoulou, A., Münz, U., Allgöwer, F., 2012. Frequency synchronization and phase agreement in Kuramoto oscillator networks with delays. Automatica 48 (12), 3008-3017.

Sepulchre, R., 2011. Consensus on nonlinear spaces. Annual Reviews in Control 35 (1), 56-64.

Sepulchre, R., Paley, D. A., Leonard, N. E., 2007. Stabilization of planar collective motion: All-to-all communication. IEEE Transactions on Automatic Con- trol $52(5), 811-824$.

Sepulchre, R., Paley, D. A., Leonard, N. E., 2008. Stabilization of planar collective motion with limited communication. IEEE Transactions on Automatic Control 53 (3), 706-719.

Shafi, S. Y., Arcak, M., Jovanović, M., Packard, A. K., 2013. Synchronization of diffusively-coupled limit cycle oscillators. Automatica 49 (12), 3613-3622.

Shim, S. B., Imboden, M., Mohanty, P., 2007. Synchronized oscillation in coupled nanomechanical oscillators. Science 316 (5821), 95-99.

Simeone, O., Spagnolini, U., Bar-Ness, Y., Strogatz, S. H., 2008. Distributed synchronization in wireless networks. IEEE Signal Processing Magazine 25 (5), 81-97.

Simpson-Porco, J. W., Dörfler, F., Bullo, F., 2013. Synchronization and power sharing for droop-controlled inverters in islanded microgrids. Automatica 49 (9), 2603-2611.

Skardal, P. S., Sun, J., Taylor, D., Restrepo, J. G., 2013. Effects of degree-frequency correlations on network synchronization: Universality and full phase-locking. EPL (Europhysics Letters) 101 (2), 20001.

So, P., Luke, T. B., Barreto, E., 2014. Networks of theta neurons with time-varying excitability: Macroscopic chaos, multistability, and final-state uncertainty. Physica D: Nonlinear Phenomena 267, 16-26.

Sonnenschein, B., Schimansky-Geier, L., 2012. Onset of synchronization in complex networks of noisy oscillators. Physical Review E 85 (5), 051116.

Sontag, E. D., 2010. Contractive systems with inputs. In: Willems, J. C., Hara, S., Ohta, Y., Fujioka, H. (Eds.), Perspectives in Mathematical System Theory, Control, and Signal Processing. Springer, pp. 217-228.

Sorrentino, F., Porfiri, M., 2011. Analysis of parameter mismatches in the master stability function for network synchronization. EPL (Europhysics Letters) $93(5), 50002$.

Strogatz, S. H., 2000. From Kuramoto to Crawford: Exploring the onset of synchronization in populations of coupled oscillators. Physica D: Nonlinear Phenomena 143 (1), 1-20.

Strogatz, S. H., 2001. Exploring complex networks. Nature 410 (6825), 268-276.

Strogatz, S. H., 2003. SYNC: The Emerging Science of Spontaneous Order. Hyperion.

Strogatz, S. H., Abrams, D. M., McRobie, A., Eckhardt, B., Ott, E., 2005. Theoretical mechanics: Crowd synchrony on the Millennium Bridge. Nature 438 (7064), 43-44.

Strogatz, S. H., Mirollo, R. E., 1988. Phase-locking and critical phenomena in lattices of coupled nonlinear oscillators with random intrinsic frequencies. Physica D: Nonlinear Phenomena 31 (2), 143-168.

Subbarao, D., Uma, R., Saha, B., Phanendra, M. V. R., 2001. Self-organization on a power system. IEEE Power Engineering Review 21 (12), 59-61.

Sun, J., Bollt, E. M., Nishikawa, T., 2009. Master stability functions for coupled nearly identical dynamical 
systems. EPL (Europhysics Letters) 85 (6), 60011.

Suykens, J. A. K., Osipov, G. V., 2008. Introduction to focus issue: Synchronization in complex networks. Chaos: An Interdisciplinary Journal of Nonlinear Science 18 (3), 037101-037101.

Tanaka, H. A., Lichtenberg, A. J., Oishi, S., 1997. Selfsynchronization of coupled oscillators with hysteretic responses. Physica D: Nonlinear Phenomena 100 (34), 279-300.

Tass, P. A., 2003. A model of desynchronizing deep brain stimulation with a demand-controlled coordinated reset of neural subpopulations. Biological Cybernetics 89 (2), 81-88.

Tavora, C. J., Smith, O. J. M., 1972a. Equilibrium analysis of power systems. IEEE Transactions on Power Apparatus and Systems 91 (3), 1131-1137.

Tavora, C. J., Smith, O. J. M., 1972b. Stability analysis of power systems. IEEE Transactions on Power Apparatus and Systems 91 (3), 1138-1144.

Tilton, A. K., Hsiao-Wecksler, E. T., Mehta, P. G., Jun. 2012. Filtering with rhythms: Application to estimation of gait cycle. In: American Control Conference. Montréal, Canada, pp. 3433-3438.

Tönjes, R., 2007. Pattern formation through synchronization in systems of nonidentical autonomous oscillators. Ph.D. thesis, Universitäts Potsdam, Germany.

Tuna, E. S., 2012. Synchronization analysis of coupled Lienard-type oscillators by averaging. Automatica 48 (8), 1885-1891.

Van Der Pol, B., 1927. Forced oscillations in a circuit with non-linear resistance. The London, Edinburgh, and Dublin Philosophical Magazine and Journal of Science 3 (13), 65-80.

Varela, F., Lachaux, J. P., Rodriguez, E., Martinerie, J., 2001. The brainweb: Phase synchronization and largescale integration. Nature Reviews Neuroscience 2 (4), 229-239.

Vassilieva, E., Pinto, G., Acacio de Barros, J., Suppes, P., 2011. Learning pattern recognition through quasisynchronization of phase oscillators. IEEE Transactions on Neural Networks 22 (1), 84-95.

Verwoerd, M., Mason, O., 2008. Global phase-locking in finite populations of phase-coupled oscillators. SIAM Journal on Applied Dynamical Systems 7 (1), 134160.

Verwoerd, M., Mason, O., 2009. On computing the critical coupling coefficient for the Kuramoto model on a complete bipartite graph. SIAM Journal on Applied Dynamical Systems 8 (1), 417-453.

Verwoerd, M., Mason, O., 2011. A convergence result for the Kuramoto model with all-to-all coupling. SIAM Journal on Applied Dynamical Systems 10 (3), 906920.

Vicsek, T., Czirók, A., Ben-Jacob, E., Cohen, I., Shochet, O., 1995. Novel type of phase transition in a system of self-driven particles. Physical Review Letters 75 (6-7), 1226-1229.

Walker, T. J., 1969. Acoustic synchrony: two mechanisms in the snowy tree cricket. Science 166 (3907),
891-894.

Wang, W., Ghosh, B., Sep. 2007. Kuramoto models, coupled oscillations and laser networks. In: SICE Annual Conference. Kagawa, Japan, pp. 130-135.

Wang, W., Slotine, J.-J. E., 2005. On partial contraction analysis for coupled nonlinear oscillators. Biological Cybernetics 92 (1), 38-53.

Wang, Y., Doyle, F. J., 2012. Optimal phase response functions for fast pulse-coupled synchronization in wireless sensor networks. IEEE Transactions on Signal Processing 60 (10), 5583-5588.

Wang, Y., Doyle, F. J., 2013. Exponential synchronization rate of Kuramoto oscillators in the presence of a pacemaker. IEEE Transactions on Automatic Control 58 (4), 989-994.

Wang, Y., Núñez, F., Doyle, F. J., 2013. Increasing sync rate of pulse-coupled oscillators via phase response function design: theory and application to wireless networks. IEEE Transactions on Control Systems Technology 21 (4), 1455-1462.

Wieland, P., 2010. From static to dynamic couplings in consensus and synchronization among identical and non-identical systems. Ph.D. thesis, Universität Stuttgart.

Wiener, N., 1948. Cybernetics; or Control and Communication in the Animal and the Machine. Wiley.

Wiener, N., 1958. Nonlinear Problems in Random Theory. MIT Press.

Wiesenfeld, K., Colet, P., Strogatz, S. H., 1998. Frequency locking in Josephson arrays: Connection with the Kuramoto model. Physical Review E 57 (2), 15631569.

Wiley, D. A., Strogatz, S. H., Girvan, M., 2006. The size of the sync basin. Chaos: An Interdisciplinary Journal of Nonlinear Science 16 (1), 015103.

Winfree, A. T., 1967. Biological rhythms and the behavior of populations of coupled oscillators. Journal of Theoretical Biology 16 (1), 15-42.

Winfree, A. T., 2001. The Geometry of Biological Time, 2nd Edition. Springer.

Witthaut, D., Timme, M., 2013. Kuramoto dynamics in Hamiltonian systems. arXiv preprint arXiv:1305.1742.

Wu, F., Kumagai, S., 1982. Steady-state security regions of power systems. IEEE Transactions on Circuits and Systems 29 (11), 703-711.

Wu, F. F., Kumagai, S., 1980. Limits on Power Injections for Power Flow Equations to Have Secure Solutions. Electronics Research Laboratory, College of Engineering, University of California.

Yin, H., Mehta, P. G., Meyn, S. P., Shanbhag, U. V., 2012. Synchronization of coupled oscillators is a game. IEEE Transactions on Automatic Control 57 (4), 920 935.

York, R. A., Compton, R. C., 2002. Quasi-optical power combining using mutually synchronized oscillator arrays. IEEE Transactions on Microwave Theory and Techniques 39 (6), 1000-1009.

Zhang, M., Wiederhecker, G. S., Manipatruni, S., 
Barnard, A., McEuen, P., Lipson, M., 2012. Synchronization of micromechanical oscillators using light. Physical Review Letters 109 (23), 233906.

Zhu, J., 2013. Synchronization of Kuramoto model in a high-dimensional linear space. Physics Letters A 377 (41), 2939-2943. 